\begin{abstract}
LI, ANG. Aligned Carbon Nanotube Sheet Piezoresistive Strain Sensors. (Under the direction of Dr. Philip Bradford).
\end{abstract}

With the application of advanced composites rapidly increasing into the aerospace and transportation industries, real time structural health monitoring (SHM) systems are attracting great attention in the composites research community. Carbon nanotubes (CNTs) have a unique set of properties that may be used for the production of a new generation strain/damage sensing materials. This research is going to introduce a novel CNT ensemble based strain sensor. The CNT sheet strain sensors were made with aligned CNT sheets and fiberglass/epoxy prepregs in a very convenient and efficient way. To evaluate the piezoresistive performance of the sensors, various types of mechanical tests were conducted with real-time electrical data acquisition. Specimens with orthogonal CNTs orientations were tested independently to investigate the influence of CNT orientation on piezoresistive behavior. The working mechanisms during mechanical straining were also discussed accordingly. Generally, the tested sensors were found to exhibit fairly good sensing stability, linearity, sensitivity and repeatability at a useful functional strain range, which is favorable for health monitoring of advanced composite material structures. It was also demonstrated that the CNT orientations have great effect on the sensitivity of sensors, leading to other promising applications such as directional strain/stress monitoring. Finally, a pre-straining process was intentionally applied, which enhanced the linearity and long-term dynamic stability of the piezoresistive sensing performance. 
(C) Copyright 2014 by Ang Li

All Rights Reserved 


\title{
Aligned Carbon Nanotube Sheet Piezoresistive Strain Sensors
}

\author{
by
}

Ang Li
A thesis submitted to the Graduate Faculty of
North Carolina State University
in partial fulfillment of the
requirements for the degree of
Master of Science

Textile Engineering

Raleigh, North Carolina

2014

APPROVED BY:

Dr. Abdel-Fattah Seyam

Committee Member
Dr. Tushar Ghosh

Committee Member

\section{Dr. Philip Bradford}

Committee Chair 


\section{BIOGRAPHY}

Ang Li was born in Xuancheng, China, May $17^{\text {th }} 1991$. He attended local middle and high schools and graduated from Jingde high school in 2008. Since he get enrolled at Anhui Polytechnic University, he left his hometown for the first time and spent 4 years of his college in $\mathrm{Wu} \mathrm{Hu}$, where he got some interest in science and engineering. Therefore, he continued his pursuit in textile engineering at the College of Textiles at North Carolina State University in 2012 fall. After the first semester, he joined the research group of Dr. Philip Bradford and has been working on composite and carbon nanotube sensors ever since. He gradually became interested in this particular area quite a lot. He is always passionate about scientific research and open to all kinds of challenges. 


\section{ACKNOWLEDGMENTS}

I would first owe my sincerest gratitude to my advisor, Dr. Philip Bradford, for his guidance and intellect in research and for always encouraging and believing in me. He has opened my eyes to the beauty of research world and helped me build a thoughtful mind and the capability to conduct scientific research independently. I am so grateful for his dedication of time and energy teaching and training me the arts of becoming a good researcher. And his revisions and comments on my thesis were definitely so crucial for the accomplishing of the whole task.

I would like to extend my thanks to other members of my committee: Dr. Tushar Ghosh and Dr. Abdel-fattah Seyam for serving on my Master Thesis Committee and their patience and precious time on reviewing my thesis.

I would also like to give my thanks to all the group members in this research group. Every one of you took a good care of me and did many favors to my research. Thank you very much.

Finally, I would like to thank my parents who can always strengthen my heart with comfort and encouraging words. You are always on my side and trust in me no matter what situations. This work would have not been possible without your reliable support. 


\section{TABLE OF CONTENTS}

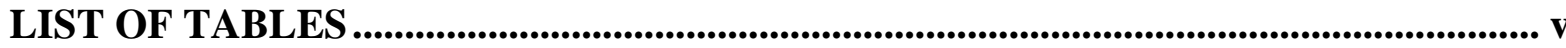

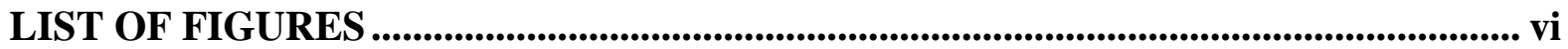

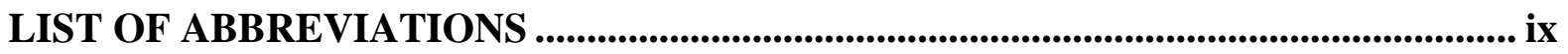

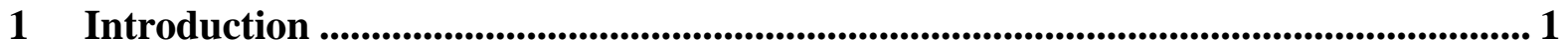

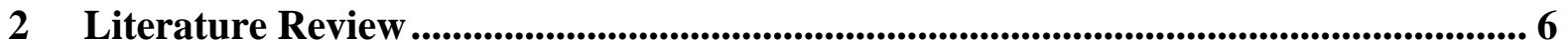

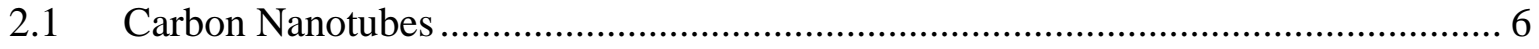

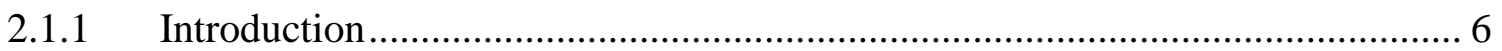

2.1.2 CNT Growth ...................................................................................... 7

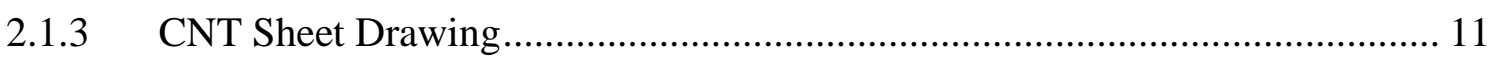

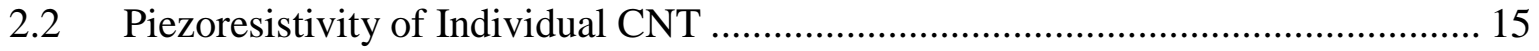

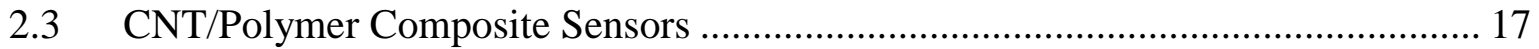

2.3.1 CNT/Polymer Hybrid Fibers...................................................................... 17

2.3.2 CNT Coated Fibers ................................................................................ 19

2.3.3 CNT/Polymer Hybrid Composite Film..................................................... 21

2.3.4 Three-dimensional CNTs Dispersed Bulk Composites ................................. 26

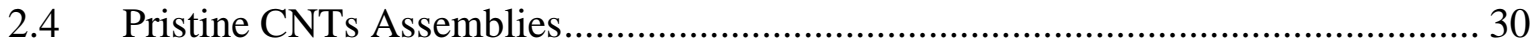

2.4.1 CNT Yarns Strain Sensor - One-dimensional Assembly .............................. 30

2.4.2 CNT Film or Bucky Paper - Two-dimensional Assembly ........................... 31

3 Experimental Procedure .............................................................................................................. 34

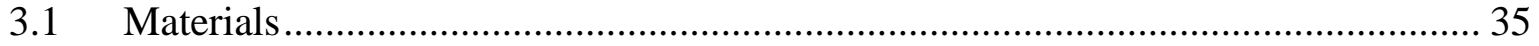

3.1.1 Multi-wall Carbon Nanotube (MWCNT) Sheet .......................................... 35

3.1.2 Fiberglass/Epoxy Prepregs....................................................................... 36

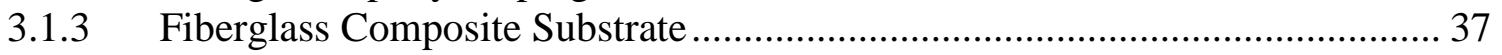

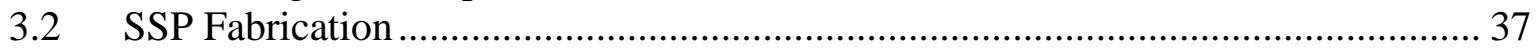

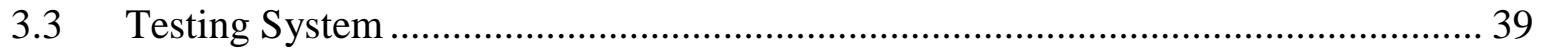

4 Experimental results and discussion.................................................................................. 43

4.1 Stability Evaluation of CNT SSPs ............................................................ 43

4.2 Sensitivity and Linearity Behavior................................................................ 47

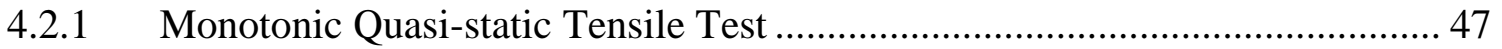

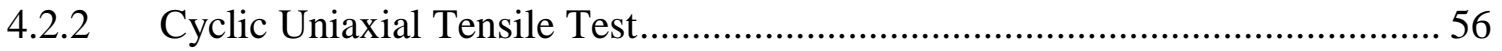

4.3 Consistency and Damage Sensing Analysis..................................................... 58

4.3.1 Progressive Damage Accumulation Test .................................................... 58

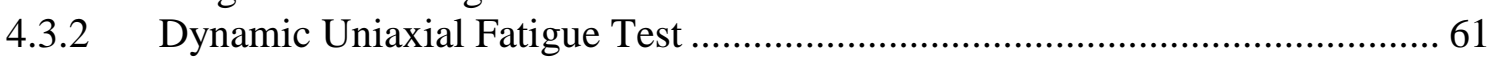

4.4 Effect of Pre-straining on Sensing Performance ........................................... 64

5 Conclusion and Future Work .............................................................................6 69

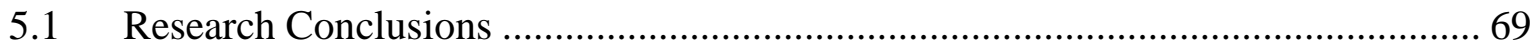

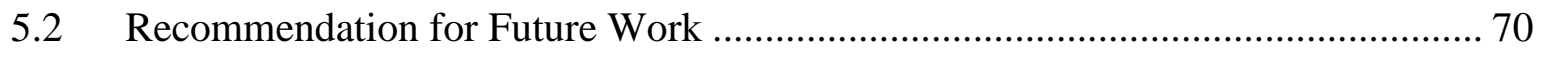

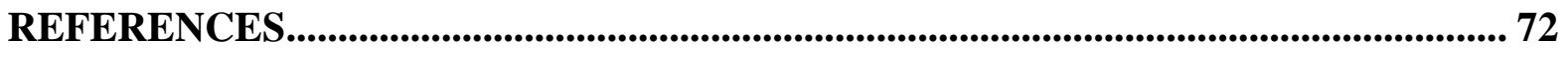

6 Appendix ............................................................................................................................. 82 


\section{LIST OF TABLES}

Table 2.1. Manufacturing and characterization of MWCNT based three-dimensional piezoresistive composite sensors with various types of polymer matrix 


\section{LIST OF FIGURES}

Figure 2.1 (a) Picture of a 2.5-mm-tall SWCNT forest on a 7-mm by 7-mm silicon wafer. (b) SEM image of 2.1-mm-long VACNTs

Figure 2.2 Scanning electron micrograph showing a draw in progress with the CNTs forming a web that recruits CNTs retained in the forest 12

Figure 2.3 SEM image of the PVA-CNT fiber (a) surface and (b) cross section, (c) Manufactured GFRP plate with embedded PVA-CNT fiber and wiring for resistance measurements 18

Figure 2.4 (a) Schematic of micro-crack bridge in epoxy composite with a MWCNT coated fiber. (b) Simultaneous change of electrical resistance and stress as a function of tensile strain

Figure 2.5 Illustration of the layer-by-layer deposition of one bilayer of a CNT-PE thin film using a self-assembly process .

Figure 2.6 (a) Schematic diagram of CNT/PDMS hybrid thin film. (b) Piezoresistive behavior of the CNT/PDMS films prepared with different volumes of nanotube dispersion .

Figure 2.7 Schematics of manufacturing of the super-aligned, transparent, flexible and stretchable SACNT/PDMS composite film 25

Figure 2.8 (a) Schematic of fabrication procedure of MWCNT/PC composite strain sensor including wet mixing and casting, (b) Schematic of the planetary mixer .

Figure 2.9 (a) SEM image of spinning CNT yarns from the as-grown CNT forest. (b) Process of Al electrodes deposition on a CNT yarn using magetron sputtering. (c) Schematic of the side and cross-section of the laminated composite sample with transversely stitched CNT yarns under simultaneous three-point mechanical and two-point electrical measurements... 31

Figure 2.10 (a) SWCNT film with insulating PVC film attached to the brass specimen. (b) Voltage changes as a function of strain in the brass specimen ....

Figure 3.1 (a) CNT sheet being drawn from the array using tweezers, (b) SEM image of the side view of the CNT sheet showing the approximate thickness and low density structure, (c) high magnification SEM image of the top of the CNT sheet showing the spacing between CNTs and (d) TEM image of CNTs from the sheet 36 
Figure 3.2 Step by step fabrication process and schematic configuration of testing specimens.

Figure 3.3 Typical mechanical tensile test set-up. (a) front surface with a CNT SSP, (b) back surface with a metal foil strain gauge. 40

Figure 3.4 Coupled mechanical-electrical test and data acquisition system. The electrical data was collected in-situ while the mechanical tensile tests were ongoing. 41

Figure 4.1 (a) Long term intrinsic resistance stability: the resistance of the SSP was measured with zero load applied at room temperature over 120 hours. (b) Electrical resistance reacted correspondingly to the temperature variation in the range of $22 \sim 100{ }^{\circ} \mathrm{C}$ and was able to keep the value at relatively high temperature over time. 44

Figure 4.2 The mechanical stress/strain curve of monotonic tensile test and normalized resistance change $\left(\Delta R / R_{0}\right)$ of parallel and perpendicular SSPs versus strain $(\varepsilon)$. The $\Delta R / R_{0}$ increases linearly at lower $\varepsilon$, then follows a non-linear behavior thereafter. The response of $\Delta \mathrm{R} / \mathrm{R}_{0}$ to $\varepsilon$ for the parallel SSP is more sensitive. 48

Figure 4.3 Constant strain cyclic tensile test: normalized electrical resistance change of parallel and perpendicular SSPs versus time in response to ten cycle loading - unloading of $0.8 \%$ strain.. 56

Figure 4.4 The real-time electrical resistance variation of parallel and perpendicular SSPs as well as stress/strain data corresponding to progressive damage loading with $7 \mathrm{kN}$ increment for each cycle until fracture. The stress was unloaded to zero after each cycle, producing residual strain and irreversible $\Delta \mathrm{R} / \mathrm{R}_{0}$ of both parallel and perpendicular SSPs. 59

Figure 4.5 Residual axial strain and corresponding irreversible resistance change of parallel and perpendicular SSPs in the progressive damage accumulation tests. A quasi-linear increase of the residual strain can be observed, generally matching with the irreversible resistance change progression. Transverse crack induced by high level strain damaged the CNTs network in parallel SSPs more significantly, leading to its curve deviation. (the cycles are identical to those in the progressive damage accumulation tests in Figure 4.4).

Figure 4.6 Relative resistance change $\left(\Delta R / R_{0}\right)$ response of (a) parallel and (b) perpendicular SSPs stretched to $0.4 \%$ (red) and $0.8 \%$ (black) strain ( $\varepsilon$ ) during 1000 cycle long term fatigue test. The $\Delta \mathrm{R} / \mathrm{R}_{0}$ behavior is stable and consistent for both types of SSPs at $0.4 \% \varepsilon$. The value of $\Delta R / R_{0}$ varied in an expanding range over time at $0.8 \% \varepsilon$, and more obvious $\Delta R / R_{0}$ peak value increase can be observed for parallel SSPs. The $\Delta R / R_{0}$ at zero load increased over time as well. A transition zone occurred after certain numbers of loading - unloading, where all the trends above were attenuated, showing a gradually stabilized piezoresistive behavior. .. 62 
Figure 4.7 Monotonic tensile test after pre-straining, showing better linearity but lower sensitivity (GF of 1$)$ of normalized resistance change $\left(\Delta \mathrm{R} / \mathrm{R}_{0}\right)$ to strain in the range of $0 \sim 0.7 \%$. Smooth transition from linear to non-linear regime showed better sensing stability of pre-strained specimens.

Figure 4.8 Comparison of the piezoresistive response of parallel SSPs without pre-straining (red) and after pre-straining to $1.2 \%$ (blue) for 1000 cycle fatigue test. The pre-strained specimens showed better stability and reversibility over long time testing, exhibiting more reliable sensing performance. Inset: close-up of the first 10 cycles of $\Delta R / R_{0}$ response to strain. 


\section{LIST OF ABBREVIATIONS}

\begin{tabular}{|c|c|}
\hline $\mathrm{Al}_{2} \mathrm{O}_{3}$ & Aluminum Oxide \\
\hline CNT & Carbon Nanotube \\
\hline Co & Cobalt \\
\hline CVD & Chemical Vapor Deposition \\
\hline DMF & Dimethylformamide \\
\hline DWCNT & Doubled-Walled Carbon Nanotube \\
\hline EPD & Electrophoretic Deposition \\
\hline FBG & Fiber Bragg Grating \\
\hline $\mathrm{FeC}_{2}$ & Iron Carbide (carbon-rich) \\
\hline $\mathrm{FeCl}_{2}$ & Iron(II) Chloride \\
\hline GF & Gauge Factor \\
\hline $\mathrm{LbL}$ & Layer-by-Layer \\
\hline Mo & Molybdenum \\
\hline $\mathrm{Ni}$ & Nickel \\
\hline MWCNT & Multi-Walled Carbon Nanotube \\
\hline $\mathrm{PC}$ & Polycarbonate \\
\hline PDA & Progressive Damage Accumulation \\
\hline PDMS & Polydimethylsiloxane \\
\hline $\mathrm{PE}$ & Polyethylene \\
\hline PEO & Polyethylene Oxide \\
\hline PLLA & Poly-L-Lactic-Acid \\
\hline PSF & Polysulfone \\
\hline PVA & Polyvinyl alcohol \\
\hline PZT & Lead Zirconate Titanate \\
\hline SACNT & Super-Aligned Carbon Nanotube \\
\hline SHM & Structural Health Monitoring \\
\hline SSP & Strain Sensing Patch \\
\hline SWCNT & Single-Walled Carbon Nanotube \\
\hline $\mathrm{THF}$ & Tetrahydrofuran \\
\hline VACNT & Vertically Aligned Carbon Nanotube \\
\hline $\mathrm{ZnO}$ & Zinc Oxide \\
\hline cpm & cycle per minute \\
\hline $\mathrm{sccm}$ & standard cubic centimeter per minute \\
\hline
\end{tabular}




\section{Introduction}

\subsection{Background}

Composites are increasing in popularity in many advanced applications including aircraft, spacecraft, automobiles, ships, and civil infrastructure [1]. Yet, damage in composites is difficult to detect because micro-cracking, delamination and fiber breakage often occur inside the material. Since those types of damage cannot be observed at the surface, internal nondestructive evaluations are in great need to identify even subtle damage inside the composites. This kind of evaluation is done periodically and not in real time during operational life span of composite. Therefore, real-time strain and damage monitoring of composite structures is a new area of research that may be critical for component maintenance as well as the prevention of catastrophic failure [2]. To fulfill this purpose, a variety of procedures using embedded or attached sensors, such as metal foil strain gauges, piezoelectric sensors (e.g., ZnO and PZT sensors) and optical fiber sensors (e.g., fiber Bragg grating sensors), have been applied in and on composites for structural health monitoring [3]. Metal foil strain gauges are the most common and conventional piezoresistive strain sensor. Such devices can reliably measure strains of up to $5 \%$ with a gauge factor between $2 \sim 3.2$ [4]. This type of low cost sensors is widely used in the SHM field. However, the capability for only local surface based sensing and the non-conformable nature limit their effective function as integral SHM devices. Piezoelectric sensors have been successfully used to monitor structural damage based on acoustic wave principles [5]. Typical PZT sensors have good performance monitoring dynamic signals but are generally more expensive. They are 
likely to be depolarized by either mechanical stress or thermal effect, which would draw some concerns on long time sensing stability. And their sensitive reaction to temperature would also cause troubles for high temperature sensing applications [6]. Optical interference signals are also sensitive to damage and strain, so fiber-optical sensors are quite good choice for composite damage monitoring considering their lightweight and electromagnetic interference insensitivity. However, limitations of fiber optics include difficulty in measuring strains in different directions at the same location and the high cost of the optical demodulator [3]. Fiber Bragg grating (FBG) sensors are able to measure the length of the delamination through spectrum changes [7] and provide a large number of low bandwidth strain measurements along one fiber optic cable [8]. Embedding of fiber Bragg grating sensors in composites should be done carefully to avoid affecting the strength of host materials. This may limit their applications in accurate sensing of some composite parts whose dimensions are small [7].

Among the variety of sensing transducers used for structural health monitoring, strain sensors rank among the most important for determining long-term structural reliability and health, as they correspond directly to the induced localized stress fields commonly used to predict the onset of damage [9]. Taking into account that all the embedded and attached sensors have their advantages and disadvantages, scientists and engineers are putting more effort to develop a new generation of strain sensors with high performance advanced materials. Most recently, the concept of using carbon nanotubes dispersed in a polymeric matrix as piezoresistive sensors was introduced to monitor strain and failure mechanisms in glass fiber 
composites [10]. A high sensitivity of this new method was demonstrated when identifying the nature and progression of matrix-dominated damage in glass fiber reinforced nanocomposites $[11,12]$. Carbon nanotubes (CNTs) have versatile mechanical properties and excellent piezoresistive properties, offering attractive alternatives for developing new generation strain sensors in multiscale. This may help to overcome the limitations of existing conventional sensors, including limited monitoring locations, fixed directions and separation from the structure that is being monitored. With continuous research and development on the CNT-based sensing materials, the goal to build a flexible and reliable system for the in-situ SHM application at a reasonable cost would be achieved in the near future.

\subsection{Motivation and Objectives}

The outstanding intrinsic properties of CNTs make them desirable for application in SHM fields [1]. Among those various attractive properties, their piezoresistive behavior makes CNT a good candidate to function as a strain sensing material. In recent years, many researchers have already investigated a diverse array of CNT-based sensors with different structures, including pristine CNTs assemblies (pure CNT yarns $[3,13,14]$ and bucky paper [15-18]) and CNT/polymer hybrid composites with random or aligned CNT dispersions (CNT fibers [19-24], films [25-46] and bulk composite [47-61]). However, the existing CNTbased strain sensors suffer from different issues which often limit their further development in practical applications. For example, CNTs yarn sensors have excellent repeatable and stable electrical resistance to strain correlate behavior, yet only very low gauge factors were reported (around 0.5 in [13]). Bucky paper CNT sensors have low strength and will fracture 
at very low strain. So they are obviously not capable for long term use, although good sensitivity had been achieved in [15]. The higher sensitivity and mechanical strength of CNTs/polymer nanocomposites mentioned in many research is encouraging. However, the problem of initiative electrical resistance drifting in zero loading condition may limit their use as real sensors. Also, resistance hysteresis in a cyclic strain testing was found in these composite sensors [12,57]. All types of CNT based strain sensors will be discussed later in the literature review section with more details covering some representative features.

The CNTs can not only serve as sensors coupled with composite materials, but also function as a reinforcing element. A highly desirable characteristic of the composite materials is to align CNTs inside the host polymer matrix, because alignment of CNTs induces anisotropy in the composite, thus improving the mechanical properties in the alignment direction [10]. In addition, many researchers claimed that good alignment of CNT assemblies is beneficial for increasing the piezoresistive effect. Recently, A.I. Oliva et al. aligned CNTs in PSF matrix by an electrical field, resulting in improved electrical and piezoresistive sensing capabilities of composite film [62]. Theodosiou and Saravanos [63] predicted that CNT alignment in the direction of the applied strain would increase the piezoresistive sensitivity of the composite based on a multiscale modeling strategy. Ultimately, our objective is to develop a type of unique CNT-based piezoresistive strain sensors which are sensitive, repeatable, robust, easy to make, cost effective and can be surface mounted or integrated internally into composites. 
As a first step toward achieving those objectives, this thesis introduces a novel aligned CNTs sheet strain sensing patch (SSP) that can be mounted to the surface of traditional composites. Continuous aligned carbon nanotube sheets were pulled directly from spinnable MWCNT arrays and able to be incorporated onto composites for strain sensing. These CNT sheets were transferred onto fiberglass/epoxy prepregs and cured altogether with the host composites. Thus, CNTs sheets can be readily incorporated onto composite structures for in situ health monitoring. The well aligned CNTs sheets are expected to reinforce the epoxy resin system, obtaining better mechanical properties. On the other hand, two groups of SSPs with orthogonal orientations were studied respectively to evaluate the effect of CNTs alignment on strain sensing performance. The piezoresistive responses of CNT SSP were investigated through various types of mechanical testing e.g., monotonic tensile testing, progressive damage accumulation tensile testing and cyclic fatigue tensile testing, demonstrating favorable damage detection and strain sensing behavior. Moreover, a substantially enhanced strain sensing performance after pre-straining stabilization was observed, making this uniquely fabricated CNTs sheet based structure very promising for strain sensing and SHM of composite materials. 


\section{Literature Review}

\subsection{Carbon Nanotubes}

\subsubsection{Introduction}

Carbon nanotubes are cylindrical nanostructures that can be conceptualized as one-atomthick layers of carbon, i.e. graphene, rolled up into a long and hollow geometry [64]. Carbon atoms are typically positioned in a repeating hexagonal shape with carbon atoms at the six

corners. The chemical bonding consists fully of strong $\mathrm{sp}^{2}$ bonds, which establish the foundation for the high mechanical strength of CNTs [65]. Nanotubes are usually categorized as single-walled and multi-walled nanotubes different amount of graphene layers rolled up together. Obviously, single-walled carbon nanotubes (SWCNTs) can be viewed as cylinders rolled up from only one single layer of graphene. The performances of single-walled carbon nanotubes are sensitive to the purity and chirality and therefore quite difficult to control. Another type of nanotube is called multi-walled nanotube. They are concentric cylinders composed of multiple layers of graphene. Multi-walled carbon nanotubes (MWCNTs) are typically larger than SWCNTs. MWCNTs are typically more defective; however, they are more economical and can be grown at relatively high purity. MWCNTs are also not subject to chirality-related limitations on intrinsic properties and are capable to withstand aggressive processing [18]. From the aspect of practicality, we will focus on the study and application of MWCNTs for strain sensor manufacturing. Long drawable MWCNTs arrays were produce in our lab for this research via a chemical vapor deposition (CVD) method. The interest of CNTs since their discovery by Iijima in 1991, is a result of the combined spectacular 
mechanical and electrical properties [9]. Mechanically, the Young's modulus and ultimate tensile strength of high quality MWCNTs can be as high as $~ 1,200 \mathrm{GPa}$ and $150 \mathrm{GPa}$, respectively [66]. The one-dimensional nanostructure provides carbon nanotubes with their high electrical conductivity along the tube direction, which has been described as nearballistic electronic transport behavior in [67]. This essentially allows CNTs to carry electronic current without heating which is beneficial for strain sensing applications.

\subsubsection{CNT Growth}

Electric-arc discharge, laser ablation, and chemical vapor deposition (CVD) are three mainstream approaches for synthesis of carbon nanotubes [68]. The CVD process is very suitable to grow vertically aligned bulk CNTs with adequate quality and also at a relatively low cost. The carbon nanotubes used in this research were produced by a modified CVD method in our lab. In most cases, this method involves metal catalyst (e.g. $\mathrm{Fe} / \mathrm{Al}_{2} \mathrm{O}_{3}$, $\mathrm{Ni}$ and Co) and hydrocarbons (e.g. acetylene or ethylene) to grow nanotubes on a substrate (e.g. silicon and quartz) in a high temperature furnace. The catalyst particles promote the dehydrogenation of hydrocarbons at high temperature, leading to the deposition of iron carbide on the surface of the catalyst particles on the substrate. It is believed that the CNT growth was initiated during the segregation of carbon-rich iron carbide $\left(\mathrm{FeC}_{2}\right)$ [69]. Then, the

nanotubes would start to grow attached to the surface, forming vertical arrays due to the inter-tube molecular forces, shown in Figure 2.1 [68]. 

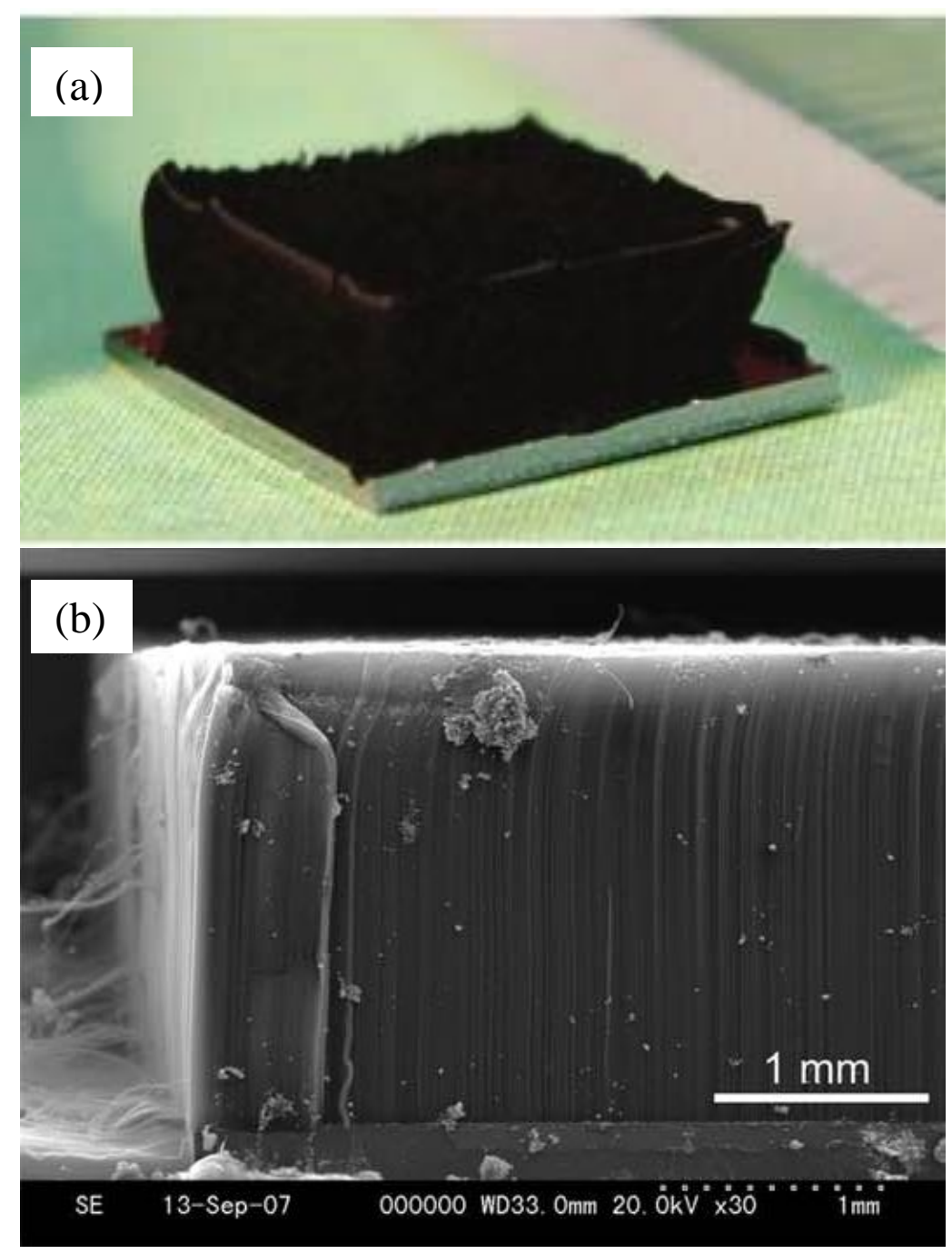

Figure 2.1 (a) Picture of a 2.5-mm-tall SWCNT forest on a 7-mm by 7-mm silicon wafer [70]. (b) SEM image of 2.1-mm-long VACNTs [69].

There are many experimental parameters influencing the stable growth of the vertically aligned carbon nanotubes (VACNTs). In recent years, many researchers have investigated various approaches to better understand and thus control the growth of CNT. L Zhang et al. [71] reported a simple method to grow vertically aligned arrays of SWCNTs via CO 
disproportionation on Co-Mo bimetallic catalysts. It was found that various forms of SWCNT arrays can be produced on the silicon substrates with Co-Mo catalyst particles been properly distributed [71]. Another effective method was developed by Zhang et al. [72] for the growth of well-aligned carbon nanotubes (CNTs) on nickel-film quartz plates by CVD process with organic ethylenediamine as a precursor. High-density carbon nanotubes were able to be vertically aligned on a large area of the quartz plates. The height of the nanotube array was able to be controlled by varying the nanotube growth time [72]. Many researchers studied the impact of the addition of water to reaction gases on CNT growth. Hata et al. [70] first demonstrated the efficient chemical vapor deposition synthesis of single-walled carbon nanotubes to enhance the activity and lifetime of catalyst via adding water vapor. More powerful water-activated catalytic activity resulted in large amount of growth of super-dense and vertically aligned nanotube forests. They normally possessed with heights more than 2 millimeters and could be easily separated from the catalysts, providing nanotube material with carbon purity above $99 \%$ [70]. Yun et al. [73] also reported that, adding water to the reaction gas mixture substantially promoted the growth which led to synthesis of longer and densely distributed CNT arrays. Using water-assisted CVD process and optimized substrate design, their group succeeded in growing vertically aligned MWCNT forest up to 4-mm in height. The arrays were of high purity and being capable to be peeled off easily from the substrate [73]. Yamada et al. [74] have even succeeded to synthesize vertically aligned doubled-walled carbon nanotube (DWCNT) forests as tall as $2.2 \mathrm{~mm}$ by water-assisted CVD method. Furthermore, catalysts were reported being able to be engineered for optimum 
DWCNT selectivity by precisely controlling the catalyst Fe film thickness. A very crucial study about the influence of mixed gas flow rates (ethylene/hydrogen) on the properties of carbon nanotubes and synthesized ultra-long carbon nanotube arrays $(\sim 1 \mathrm{~mm})$ was conducted by Zhang et al. [75]. By adjusting the ethylene and hydrogen flow rates to a proper level, the wall number, crystallinity of the graphene sheets, and length of CNTs are able to be greatly influenced. It is discovered that small ethylene flow or large hydrogen flow tends to synthesize CNTs with small diameters and few walls, and even single- and double-walled CNTs could be prepared by tuning the flow rates. These results indicate that that accurate regulation of carbon source gas and hydrogen flow rate easily enables selective synthesis of various types of CNTs arrays [75].

Research on cost-effective CNT array growth methods with cheap precursors and simple procedures has recently drawn a lot of attention. Inoue et al. [69] developed a one-step synthesis method of vertically aligned ultra-long multi-walled nanotubes using iron chloride powder as the catalyst. As claimed in their work, they were able to grow 2.1-mm-long bulk nanotubes on bare quartz surface with the low pressure CVD method and only acetylene gas flow in only 20 minutes. Moreover, the as grown CNTs were highly drawable even with such high growth rate, which also proved the high quality of the as synthesized carbon nanotubes. This method requires no additional process for catalyst preparation and only requires iron chloride powder and acetylene gas. It was found that high dehydrogenation activity of iron chloride on acetylene increases the growth rate of CNTs compared to conventional predeposited metal catalysts [69]. The extreme simplicity of this CVD process makes it a cost- 
effective method for MWCNT production from then on. The nanotube arrays used to make CNT sheet for this study were grown to a height of $\sim 1 \mathrm{~mm}$ by using a similar customized method [76], which possesses good nanotube alignment and drawability at the same time. In general, chemical vapor deposition is advantageous for achieving uniform nanotube distribution, good alignment and extremely high aspect ratio CNTs but often result in tubes that are not perfectly straight and can have more defects.

\subsubsection{CNT Sheet Drawing}

The unique feature of some vertically aligned CNT array grown by CVD method is that a unidirectional thin layer of parallelly aligned pure CNTs sheet can be directly pulled out from it in solid state [77]. This is a very rare property that can only be replicated by a few research groups in the world.

The unique drawing mechanism for such processing can be described as whenever the CNTs at the edge of the forest are pulled out they bundled or entangled with each other to form a continuous strand [78]. With the drawing process going on, subsequent CNTs in the forest were pulled out to form a "fibril" with the formerly drawn CNTs and the process repeats, shown in Figure 2.2. The fact that the CNTs assemble in this way is the basis for direct use as a sheet or for the introduction of twist to form yarns [78]. 


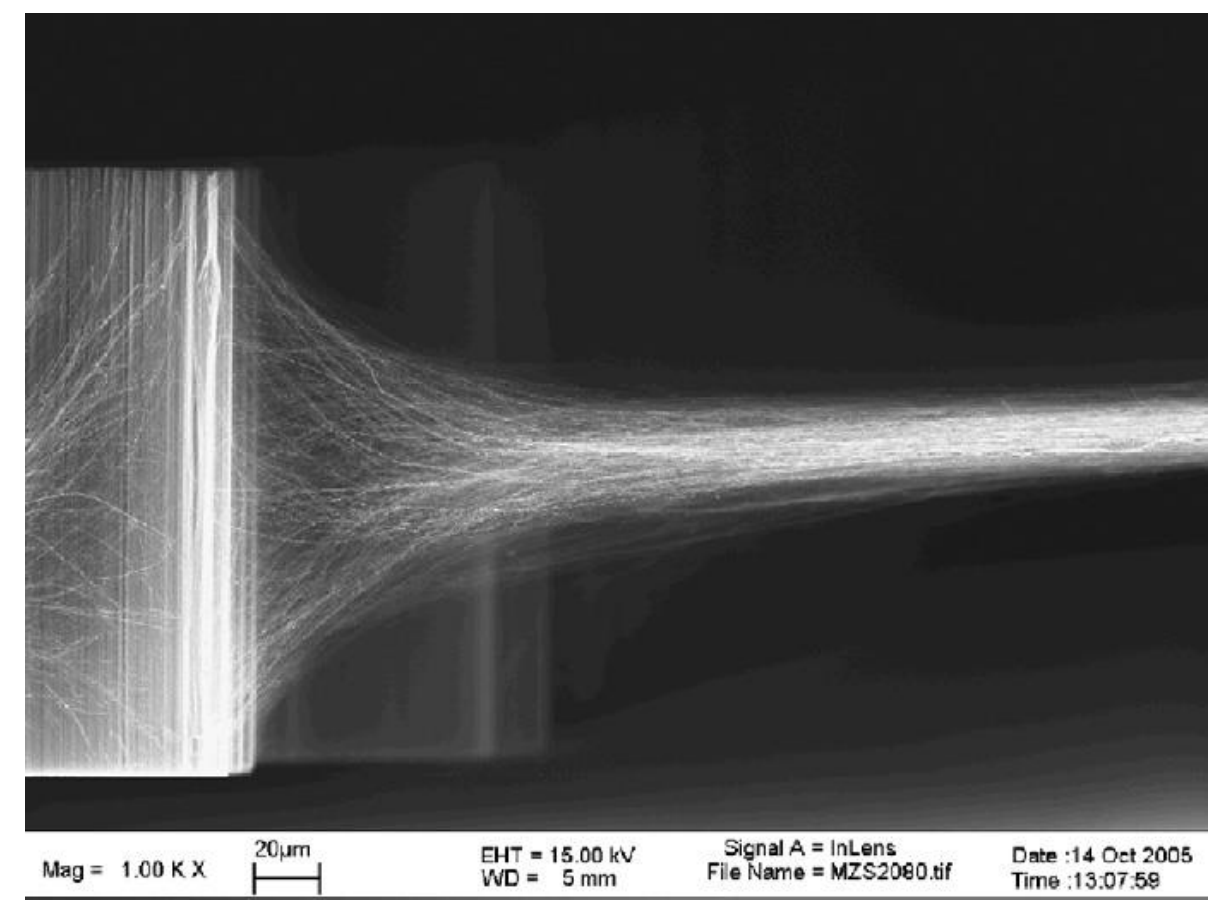

Figure 2.2 Scanning electron micrograph showing a draw in progress with the CNTs forming a web that recruits CNTs retained in the forest [78].

The drawability of vertically aligned CNT arrays is significant because it enables the CNTs to form pure assembly with uniform distribution and good alignment. On the other hand, this method of assembling the CNTs avoid the issues of nanotube properties degradation caused by chemical residual contamination and nanotube length reduction in some chemical bath and physical dispersion methods [78]. As shown in Figure 2.2, the tight bundles in CNTs sheet drawn from vertically aligned arrays indicate that strong van der Waals forces are playing an important role between the interactions of neighboring tubes. And the nanotubes in aligned CNT forests are observed to have very clean surfaces, favoring the strong interactions between nanotubes. Thus, it is concluded that the inter-tube van der Waals force 
is the main factor that makes the CNTs join end to end, forming a continuous sheet [79]. It is found by Q Li et al. that 0.5 to $1.5 \mathrm{~mm}$ long CNT arrays grown at temperatures $\leq 750{ }^{\circ} \mathrm{C}$ for 10-15 min without water vapor are favorable for spinning [80]. Too long CNT arrays were not conducive to spinning because of their relatively high content of amorphous carbon, making the nanotube surface unclean and thus fewer interactions between tubes. Good CNT alignment was found very beneficial to CNT sheet drawing. The experimental results also showed that the long CNT arrays may be highly potential to spin strong fibers. It is observed in their experiments that the highest tensile strength of the CNT fibers spun from the $1 \mathrm{~mm}$ array is $3.3 \mathrm{GPa}$, much higher than that of CNT fibers from $0.65 \mathrm{~mm}$ array (1.91 GPa) [80]. This type of technique also stimulate the research on the strain and damage sensing capability of CNT yarns, since they are both small to be embedded non-invasively into composite structure and strong to function as reinforcing element.

In order to take the advantage of the good nanotube alignment, the CNT sheets were directly utilized in CNT sensor fabrication. Although the nanotubes are resembled only by the intermolecular forces, the CNT sheet displays relatively good mechanical properties, with a tensile strength of 7.9 MPa and Young's modulus of $310 \mathrm{MPa}$ reported in the literature. The electrical conductivity of the CNT sheet exhibits semiconducting behavior [81]. After being made into fibers via spinning, even higher mechanical properties can be achieved. The tensile strength and stiffness of some CNT fibers were measured in the range of 1.35 to $3.3 \mathrm{GPa}$ and 100 to $263 \mathrm{GPa}$, respectively. Therefore, the ultrahigh specific strengths and stiffness can be achieved, coupled with an extremely low density of $(0.2 \pm 0.01) \mathrm{g} \mathrm{cm}^{-3}$ [82]. The high strength 
of CNT fibers was attributed to the long length of CNTs array. The CNT fiber strength is expected to continuously increase with even longer arrays and eventually reach a saturation value. The conclusion claimed in this work is that mechanical properties can be significantly improved by using very long CNT arrays and by improving the packing density of CNTs in the fiber [82]. This enlightened our research on using ultra-long MWCNTs sheets to make strong sensing material with multifunctional purpose e.g. mechanical reinforcement.

The CNT sheet produced from a longer array consists of larger bundles will have significantly more inter-tube contact points, providing sufficient amount of inter-tube pathways for electron transport. Electron transport at these contact points would potentially cause electric field of radiation in random directions, which breaks the induced polarization effectively. Generally, the sheet resistivity, transmittance and polarization degree can be reduced with increasing array length [77].

The good alignment, two-dimensional structure of the MWCNT sheet and extensive tube to tube overlaps between individual tubes are promising features for effective heat transport. Randomly deposited nanotubes assemblies do not provide proper pathways for the transportation of electrons and phonons. As our aligned CNT sheets are composed of many nanotube bundles with individual CNTs closely contact with each other, high degree of internanotube overlapping can be achieved. This unique feature is able to substantially decrease the electrical and thermal interconnection resistance, so as to improve the thermal conductivity of the nanotube sheets. Therefore, the high surface area and negligible heat 
capacitance make it a perfect material for sensing applications with efficient heat dissipation [83].

In summary, the well aligned CNT sheet assemblies readily drawn from the vertically aligned CNT arrays can effectively unite the nanoscopic individual CNTs, contributing to highly improved mechanical, electrical and thermal properties in macro-scale. Thanks to the unique structure of CNT sheets, they are expected to be useful in the development of well functional macroscopic CNTs sensing applications.

\subsection{Piezoresistivity of Individual CNT}

The structure of the CNT has significant impact on both the mechanical and electrical properties. It is reported that the band gap of single-walled carbon nanotubes is dependent on the variation of diameter. An increase in diameter leads to a decrease in the band gap [84]. Therefore, the mechanical deformation of CNTs can alter the band structure of carbon nanotubes as the dimensions changed correspondingly. The change of band gap will cause the variation of inter-atomic distance, making it harder or easier for the electrons to be raised to conduction band depending on the external load applied. This phenomenon will lead to a change in material conductivity and thus resistivity. This correlation between strain and resistivity can be linear with certain strain range, obtaining a constant coefficient for this behavior. This intrinsic piezoresistivity of carbon nanotubes can be very useful in the application of strain sensors. 
As discussed above, when isolated CNTs are subjected to mechanical strain, bad gap and structural changes occur, leading to significant changes in the electrical properties. In the results obtained by Tombler at [85], it was observed that under bending strain of $3.2 \%$, the conductance of the SWCNTs reduced by up to two orders of magnitude. Stampfer, et al. [86] isolated and suspended individual SWCNTs over a metallic cantilever support to investigate nanotube's potential for nano-electromechanical displacement sensing. Their results indicate a nonlinear and super sensitive piezoresistive behavior between the electrical resistance change and applied strain deformation based on the band gap changes; yet, the nonlinearity can be effectively simulated via a thermal activated transport model. As far as we know, not any previous work on piezoresistivity study of individual MWCNTs can be found in the literature. Due to their much larger diameter, multiple shell structure and commonly formed surface defects, the sensitivity for MWCNTs is speculated to be likely much lower than that of SWCNTs. The results obtained by Stampfer et al. [86] and Tombler et al. [85] shed some light on the advantages of CNT utilization for developing high-performance sensing devices. Yet, there are still realistic problems for the nanoscale fabrication of CNT sensors based on individual CNTs, including the inherent structural and electronic difference of the assynthesized CNT and difficulty to apply precise manipulation and orientation to an individual nanotube. Individual CNTs are difficult to align on a large scale and have limited maximum length by the nano-material nature. Also, the fracture of a single CNT will directly result in the failure of the device itself [87]. Thereby, it is currently not that practical to use individual CNTs as strain sensors for sensing applications that have many utilization requirements. 
To date, the main challenge for CNT strain sensing is determining how to scale geometry and sensing properties up to meet the requirements of multiple engineering fields. Different researchers have attempted to manufacture CNTs ensemble piezoresistive sensors for various applications. Given their advantages in manufacturing, the major disadvantage is the far lower sensitivity as compared to individual nanotube sensors. Failing to inherit the ultrasensitive piezoresistive behavior, the ensemble-based sensors are still superior in other sensing performance e.g. good linearity, temperature stability, reversible sensing and dynamic consistency. Depending on the fabrication details, the gauge factor for different types of CNTs ensemble piezoresistive sensors has been reported in the range from less than 0.5 [13] to 30.1 [46]. So far, there is still a relative lack of systematic studies on the diverse sensing behavior of various CNTs ensemble sensors. In order to get an idea of those macroscale CNTs ensemble piezoresistive strain sensors, we will review recent research in which the typical approaches were applied, showing some representative results and discuss the behavior.

\subsection{CNT/Polymer Composite Sensors}

\subsubsection{CNT/Polymer Hybrid Fibers}

Alexopoulos et al. demonstrated polyvinyl alcohol (PVA)/CNT hybrid fibers as the embedded element for SHM of composite structures [19-21]. The PVA/CNT fiber was produced by injecting an aqueous CNT dispersion into a co-flowing stream of a coagulating PVA solution. Figure 2.3(a) and (b) show typical scanning electron micrographs of the PVACNT fiber, exhibiting surface roughness and the homogeneous distribution of the CNTs in 
the fiber's cross-section area, respectively. As shown in Figure 2.3(c), a PVA/CNT fiber was embedded between the plies of glass fiber reinforced polymer (GFRP) composites before vacuum resin infusion. The cyclic loading test of the pre-stretched PVA/CNT composite showed that the gauge factor was $\sim 2$, with a parabolic or an exponential piezoresistive behavior to strain. The results also demonstrated that the addition of the fiber sensor did not harm the composite integrity. The observed residual electrical measurements of CNT fiber reflected possibly self-damage corresponding to almost $45 \%$ of fracture stress of the material. Thus, not only were the PVA-CNT fibers able to serve as a strain sensor for real-time sensing, but also capable of predicting the structural failure by residual electrical signals collected.

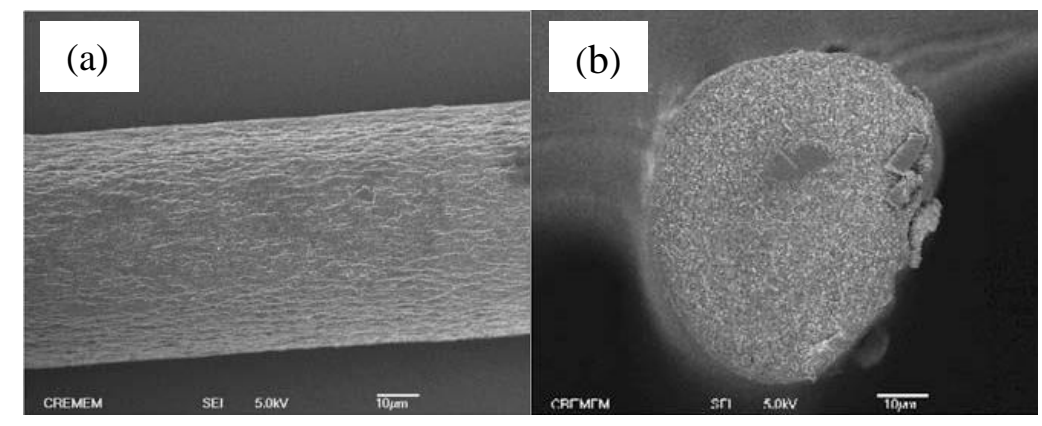

(c)

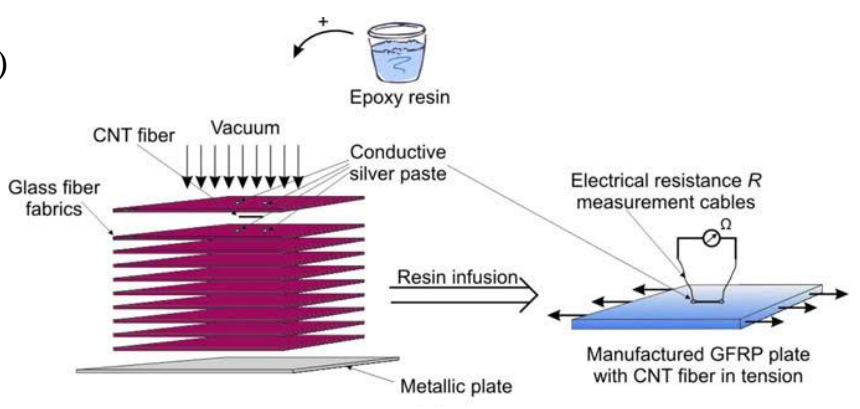

Figure 2.3 SEM image of the PVA-CNT fiber (a) surface and (b) cross section, (c) Manufactured GFRP plate with embedded PVA-CNT fiber and wiring for resistance measurements [19] 


\subsubsection{CNT Coated Fibers}

Instead of introducing another type of fiber by a different manufacturing method, Zhang et al. coated glass fibers with MWCNTs functioning as the sensing elements to predict the fracture of the reinforcing fibers in composite structures [24]. To coat the fiber, the electrophoretic deposition (EPD) technique was used to drive the negative charged CNTs towards the anode through glass fibers. As observed in their research, nanotubes were found penetrated into space between fibers and coagulated on the fiber surface, achieving a good adhesion to the glass fibers (Figure 2.4(a)). After the CNTs were applied onto the surface of glass fibers, they would then be embedded into epoxy resin. Due to the good contact between the nanotubes and the glass fibers, the breakage of the fibers would effectively induce the entire or partial fracture of CNTs coated. As shown in Figure 2.4(b), after evaluating the sensing performance, a combined linear and non-linear piezoresistive responses were discovered before the structures fracture at lower strain level. Then, a significant electrical resistance increase occurred suddenly showing the fracture of the nanotube network coated on the glass fiber surface. In conclusion, this clear three-stage piezoresistive performance of this typical structure can be used to effectively predict the fiber breakage which is invisible from the composite surface. 


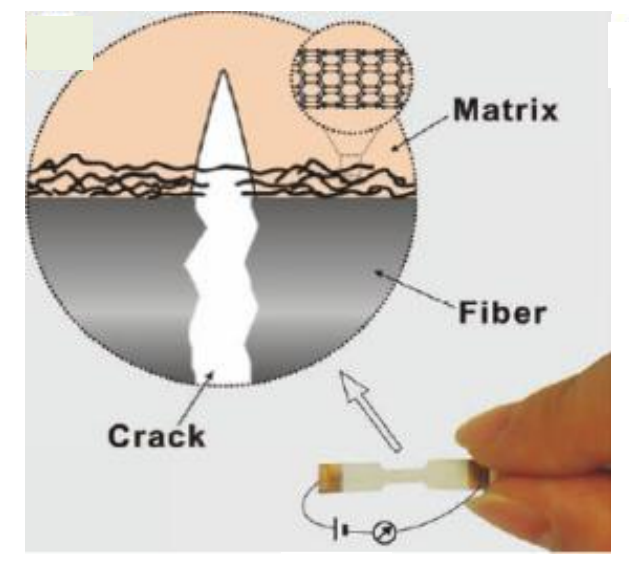

(a)

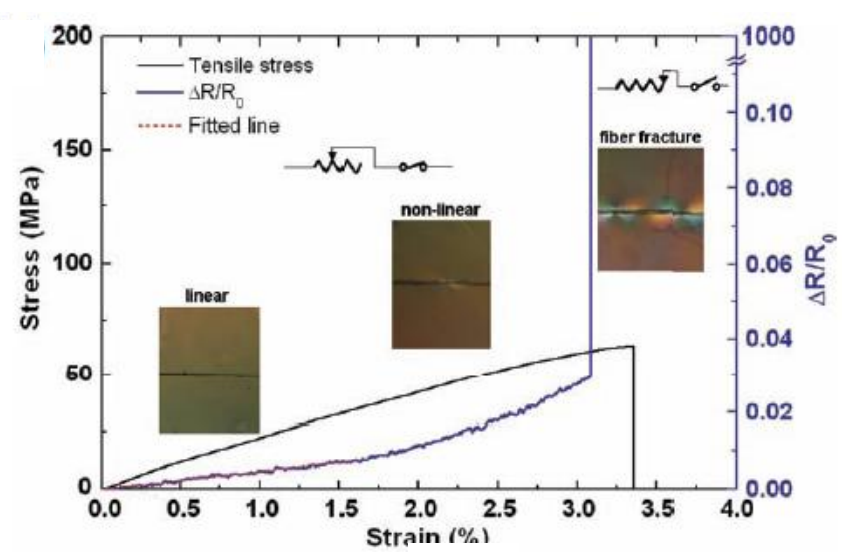

(b)

Figure 2.4 (a) Schematic of micro-crack bridge in epoxy composite with a MWCNT coated fiber. (b) Simultaneous change of electrical resistance and stress as a function of tensile strain [24].

Another novel CNT coated one-dimensional sensor has been shown by Kang et al., in which SWCNTs were coated on common cotton yarns for potential use in electrical textile area [22]. Nanotubes were first dissolved and dispersed into the solution, following which dipping and drying processes were applied to coat the cotton yarns with SWCNTs. Due to the porous structure of cotton yarn, it swelled with large amounts of the SWCNT dispersion very quickly. Interestingly, this type of sensors showed high but negative gauge factor (-24) as reported. This could possibly be explained by the transverse contraction induced by the longitudinal stretching of cotton yarns. In this case, the electrical contact area between fibrils would be enhanced significantly and result in a reduction in electrical resistance through the CNT conductive network. Due to the pretty high sensitivity gained in this study, this type of materials can be used as strain sensors and also made into smart fabrics for multiple uses in textile industry. 
CNT/polymer hybrid fibers and CNT coated fibers are both one-dimensional CNT/polymer composite sensors. As compared to nano-scale individual CNTs, the orientation and location of the composite fibers are easier to manipulate due to the attained macroscopic dimensions. In addition, they are still small in size and can be readily embedded into larger composite structures for strain and damage sensing.

\subsubsection{CNT/Polymer Hybrid Composite Film}

These types of sensors have been more widely investigated, because the two-dimensional sensors can be more compatible for multi-direction and multi-location use compared to onedirectional sensing materials. The assemblies of large amount of nanotubes provide significantly large inter-nanotube connections, leading to the formation of sufficient conductive pathways for electron conduction. Accordingly, the piezoresistive behavior of CNT/Polymer film would be more reliable for long time use and wider strain range than that of individual nanotubes and one-dimensional sensors. Moreover, they still can be readily mounted onto the surface of host materials/structure of different geometries without deteriorating the mechanical integrity and can even impart additional functionalities. However, these small film sensors can only manage local strain/stress monitoring.

K. J. Loh et al. [35] fabricated a carbon nanotube-polyelectrolyte composite multilayer thin film by a layer-by-layer (LbL) method, shown in Figure 2.5. In that research, a diverse suite of CNT-PE thin films composed of varying combinations of types of CNT, types of PE, and CNT concentration are fabricated. It was concluded that with increasing CNT concentration, one can progressively increase the strain sensitivity of the macro-scale multilayer film [35]. 
This LbL fabrication technique is pretty flexible to make films with larger thickness and therefore higher strength. Yet, a tradeoff exists between higher mechanical strength (thicker films) and lower strain sensitivity. On the other hand, these CNT and polymer hybrid film suffered from the resistance drifting at zero loading, probably due to the self-heating effect and deterioration of CNT/Polymer interfaces. Under simultaneous monotonic tensile tests and two-point electrical measurements, a linear piezoresistive behavior of the composite film loaded on an aluminum specimen was observed and the gauge factor was up to 4.5 [35].

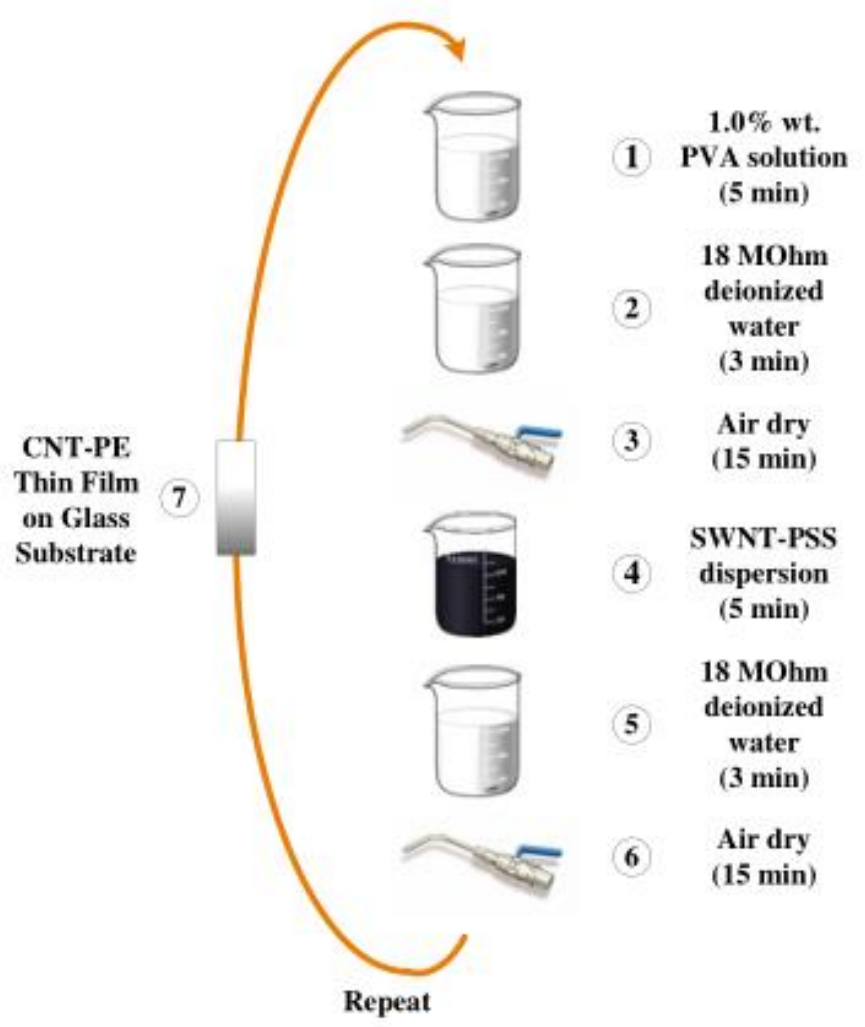

Figure 2.5 Illustration of the layer-by-layer deposition of one bilayer of a CNT-PE thin film using a self-assembly process [35]. 
MWCNT/PDMS hybrid film sensors were fabricated by Song [42] and their group by using vacuum filtration and molding transfer techniques. Once the MWCNTs suspension was made by sonication method, they would be vacuum filtrated into the mold with highly uniform nanotube dispersion. In order to enhance the adhesion of the silver paste and prevent surface contamination, another thin layer of PDMS was applied as a package covering the CNT film, after the film was formed and transferred to the PDMS substrate shown in Figure 2.6(a). The corresponding piezoresistive behavior is demonstrated in Figure 2.6(b), which exhibited a clear sensitivity variation with strong thickness dependence. As a result, the thicker the film, the higher the sensor gauge factor as illustrated by the slope of the curves [42]. The underlining physical mechanism for the observed phenomena was not clear. Even though the original studies speculated that a better load transfer between thicker CNT network and the PDMS matrix may play a role, the best gauge factor they reported was $\sim 1$ which is even lower than conventional metal foil strain gauges. Without firm experimental evidence and additional study of the mechanisms, the low piezoresistive sensitivity nature still cannot be reasonably explained which limit the application of this type of sensors to some extent. 


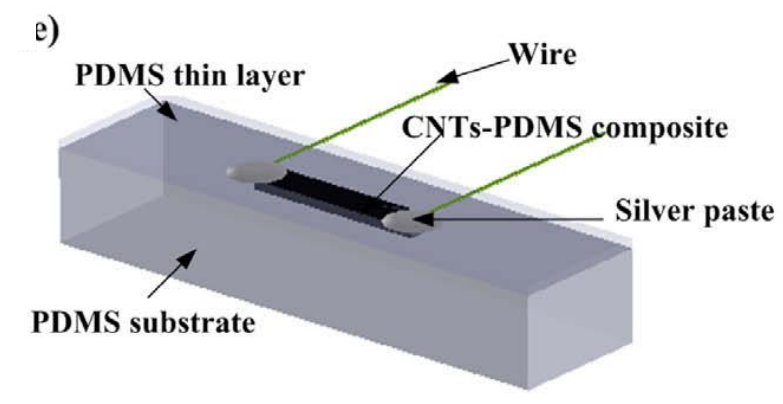

(a)

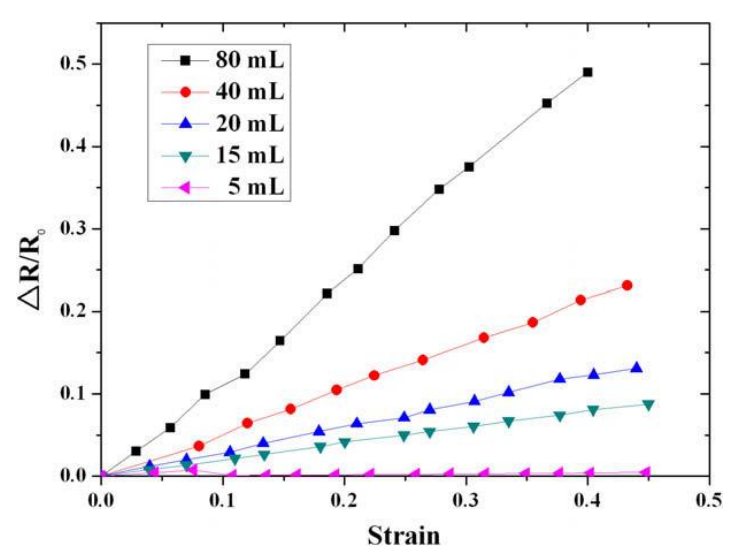

(b)

Figure 2.6 (a) Schematic diagram of CNT/PDMS hybrid thin film. (b) Piezoresistive behavior of the CNT/PDMS films prepared with different volumes of nanotube dispersion [42].

Different from the utilization of the easy-to-achieve randomly distributed CNT/polymer films, K. Liu et al. recently developed multi-layer stacked aligned transparent CNT films for strain sensing [88]. The super-aligned CNT (SACNT) film described in their research work was drawn out from a $190 \mu \mathrm{m}$ tall CNT forest grown by chemical vapor deposition method. Multiple layers were laid one by one following a pre-designed orientation (Figure 2.7(a)) and densified through a dipping process (Figure 2.7(b)). The as fabricated CNT films were further embedded into a flexible PDMS composite structure shown in Figure 2.7(c). The gauge factor of the composite sensor with a $\left(45^{\circ}, 45^{\circ}\right)$ stacking orientation was $\sim 1.17$. Much better conductivity (resistivity $\sim 10^{-4} \Omega \mathrm{cm}$ ) of aligned CNT sensors could be attained when compared to randomly distributed CNT/polymer composites. This unique feature may favor their applications in smart electronic device fields. This sensor is the most similar to the work presented here with the main differences being that they made cross-plied structures (no 
unidirectional) and they used a soft material as the matrix allowing for high deformation for flexible substrates but less applicability to structural composite materials.
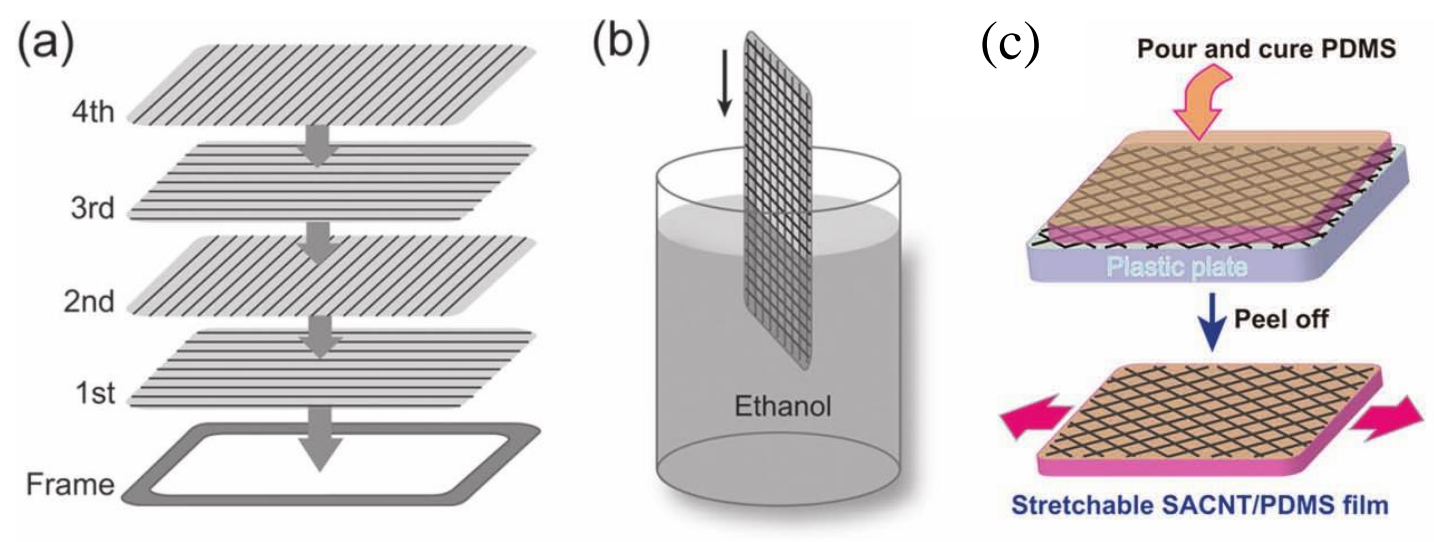

Figure 2.7 Schematics of manufacturing of the super-aligned, transparent, flexible and stretchable SACNT/PDMS composite film [88].

Generally speaking, two-dimensional sensors are more feasible and reliable to handle differernt load conditions for longer time due to the abundant CNT conductive network. This two-dimensional isotropic geometry also favored multi-direction and multi-location testing at the surface of target composite structures. In addition, mechanical integrality of the host structure would not be degraded if the film materials were properly selected and correctly engineered. However, the performance stability can not be garanteed yet, because the results from different work varied substantially recording the gauge factors from less than 1 to $\sim 10$. 


\subsubsection{Three-dimensional CNTs Dispersed Bulk Composites}

The former reviewed strain sensors can be readily incorporated with the composite in a way either into the structures or onto the surfaces of the structures. Nevertheless, they have obvious drawbacks that only local sensing is measurable. Such a problem could be overcome by directly integrating CNTs into the polymer matrix system of composite structures, also realizing many other functions at the same time. First, the CNTs are able to serve as a reinforcement for composite matrix when evenly distributed. Second, when the nanotubes are dispersed three-dimentionally in the composite, global sensing can be easily realized. Finally, with the surface modification of CNTs [89], better CNT/polymer interface bondings can be obtained, which will probably alleviate the the inter-nanotube slippage and enhance the strain sensing sensitivity. However, we should also understand the challenges acompanied with the fabrication process for the CNT dispersed bulk nanocomposites. The biggest limitation is that it is difficult to uniformly disperse the CNTs in the polymer matrices [90], which may cause problems in achieving repeatable performance of the sensor. Non-uniform disperstoin of nanotubes would even weaken the matrix in extreme cases, not to mention strong bonding and higher sensitivity. In spite of the strict requirement on fabrication processes, this approach for producing CNT based strain sensors has drawn the most attentions and efforts with large amount of associated research work done and going on. We cannot get into them in that much details. But 
Table 2.1 summarizes typical manufacturing and characterization information for studies this category of piezoresistive composite sensors with MWCNT incorporated in different types of polymers of versatile properties.

Table 2.1. Manufacturing and characterization of MWCNT based three-dimensional piezoresistive composite sensors with various types of polymer matrix

\begin{tabular}{|c|c|c|c|c|c|c|}
\hline Author & $\begin{array}{c}\text { CNT/Polymer } \\
\text { Type }\end{array}$ & $\begin{array}{c}\text { Polymerization } \\
\text { Techniques }\end{array}$ & Solvent & $\begin{array}{l}\text { Gauge } \\
\text { Factor }\end{array}$ & $\begin{array}{l}\text { Strain } \\
\text { Level }\end{array}$ & $\begin{array}{c}\text { Linearity } \\
\text { of GF }\end{array}$ \\
\hline Z. Dang [91] & $\begin{array}{l}\text { MWCNT/VMQ } \\
\text { Silicone rubber }\end{array}$ & $\begin{array}{l}\text { Wet mixing \& } \\
\text { Casting }\end{array}$ & $\begin{array}{c}\text { THF } \\
\text { (tetrahydrofuran) }\end{array}$ & N/A & N/A & Linear \\
\hline A. Oliva [62] & $\begin{array}{l}\text { MWCNT/PSF } \\
\text { (polysulfone) }\end{array}$ & $\begin{array}{l}\text { Wet mixing \& } \\
\text { Casting under } \\
\text { electric field }\end{array}$ & $\mathrm{CHCl}_{3}$ (chloroform) & $\begin{array}{l}0.7- \\
2.78\end{array}$ & $<1.5 \%$ & $\begin{array}{l}\text { Quasi- } \\
\text { linear }\end{array}$ \\
\hline Bautista [25] & MWCNT/PSF & $\begin{array}{l}\text { Wet mixing \& } \\
\text { Casting }\end{array}$ & $\mathrm{CHCl}_{3}$ & 0.48 & $<1 \%$ & Linear \\
\hline Y. Liu [46] & $\begin{array}{l}\text { MWCNT/PLLA } \\
\text { Poly(L-lactide) }\end{array}$ & $\begin{array}{l}\text { Wet mixing \& } \\
\text { Casting }\end{array}$ & $\mathrm{CH}_{2} \mathrm{Cl}_{2}$ & $\begin{array}{l}8.6- \\
30.1\end{array}$ & $<0.5 \%$ & Linear \\
\hline $\begin{array}{l}\text { W. Zhang } \\
\text { [45] }\end{array}$ & $\begin{array}{l}\text { MWCNT/PC } \\
\text { polycarbonate }\end{array}$ & $\begin{array}{l}\text { Wet mixing \& } \\
\text { Casting }\end{array}$ & THF & 7 & $<1.68 \%$ & Linear \\
\hline C. Gau [92] & MWCNT/PI & $\begin{array}{l}\text { Wet mixing \& } \\
\text { Spin Coating }\end{array}$ & $\begin{array}{c}\text { DMAc } \\
\text { dimethylacetamide }\end{array}$ & 1.27 & N/A & Nonlinear \\
\hline Hwang [93] & MWCNT/PDMS & $\begin{array}{l}\text { Wet mixing \& } \\
\text { blade coating }\end{array}$ & $\begin{array}{l}\mathrm{P}_{3} \mathrm{HT} \text { poly }(3- \\
\text { hexylthiophene }\end{array}$ & N/A & N/A & $\begin{array}{c}\text { Linear } \\
\text { Nonlinear }\end{array}$ \\
\hline C. $\mathrm{Hu}[94]$ & MWCNT/PDMS & $\begin{array}{l}\text { Wet mixing \& } \\
\text { Casting }\end{array}$ & Ethyl acetate & N/A & N/A & Nonlinear \\
\hline $\begin{array}{c}\text { N. Hu } \\
{[31,95,96]}\end{array}$ & $\begin{array}{l}\text { MWCNT/bishe } \\
\text { Nol-F epoxy \& } \\
\text { hardener }\end{array}$ & $\begin{array}{l}\text { Planetary } \\
\text { mixing \& } \\
\text { Casting }\end{array}$ & No & $6.4-22.4$ & $<0.6 \%$ & Nonlinear \\
\hline M. Park [39] & $\begin{array}{c}\text { MWCNT/PEO } \\
\text { polyethylene } \\
\text { oxide }\end{array}$ & $\begin{array}{l}\text { Wet mixing \& } \\
\text { Casting }\end{array}$ & N/A & N/A & $<7 \%$ & $\begin{array}{l}\text { Linear \& } \\
\text { Nonlinear }\end{array}$ \\
\hline $\begin{array}{l}\text { Thostenson } \\
{[11,12,50]}\end{array}$ & $\begin{array}{c}\text { MWCNT/epoxy } \\
\& \text { amine } \\
\text { hardener }\end{array}$ & $\begin{array}{l}\text { 3-roll-mill } \\
\text { calendering \& } \\
\text { VARTM }\end{array}$ & No & N/A & $<2 \%$ & Nonlinear \\
\hline
\end{tabular}


Apparently seen form the table, wet mixing and casting techniques are the most widely used techniques for nanotube dispersion for three-dimensional CNT/polymer bulk composite manufacturing. For example in W. Zhang's work [45], with MWCNTs dispersed and polycarbonate (PC) solved in tetrahydrofuran (THF) respectively, the two portions of solution were then mixed together and sonicated to ensure good mixing. Finally, the methanol (anti-solvent of PC) was used to precipitate the MWCNT/PC composite, which was then filtered out and dried in a vacuum oven (Figure 2.8(a)). The piezoresistive sensitivity observed was $~ 3.5$ times that of typical metal foil strain gauges, which served as a reference in their experiment. Besides these most frequently utilized methods, Gau et al. demonstrated a spin coating technique in their research work to spread compound solutions with dispersed MWCNTs onto a glass substrate before curing [92]. In comparison, the specimens made through this method showed much less sensitive sensing performance than that in $\mathrm{W}$. Zhang's work using wet mixing and casting process. As shown in Figure 2.8(b), N. Hu et al. used a novel dry mixing technique (a planetary mixer) to directly mix MWCNT, epoxy resin and the hardener altogether [31]. This method is highly controlled by merely adjusting the speed of the mixer, achieving different level of CNTs dispersion. In this research, they compared the piezoresistive sensing behavior of specimens made with different mixing speed. Higher stirring or mixing rate is suggested to improve the sensitivity possible due to the fast mixing induced good dispersion of nanotubes and thus good conductivity. 
(a)
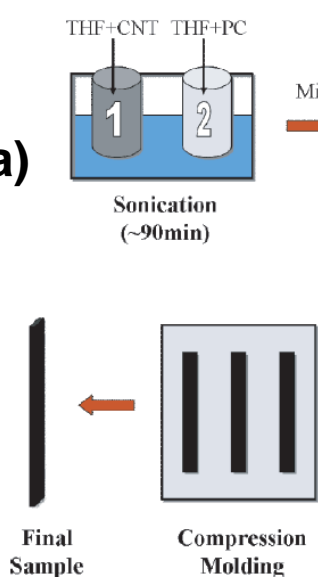
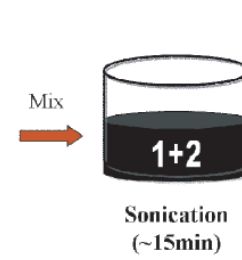

4

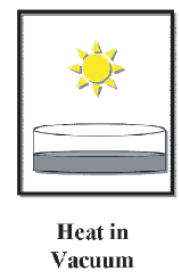

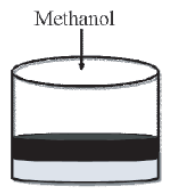

Precipitation
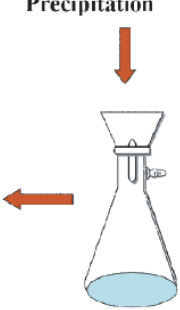

Filtration

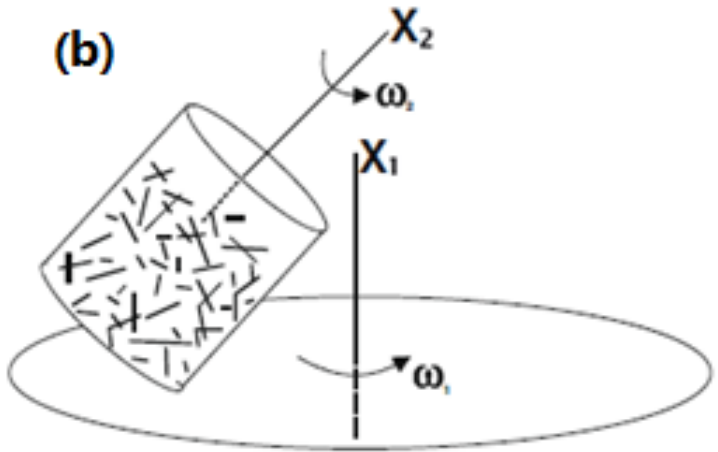

Figure 2.8 (a) Schematic of fabrication procedure of MWCNT/PC composite strain sensor including wet mixing and casting [45], (b) Schematic of the planetary mixer [31].

Thostenson and his group also did CNT infusion to glass fiber composite combined with some sort of physical treatment to achieve good nanotube distribution. They have achieved great success on the carbon nanotube network strain sensing embedded in advanced glass fiber-epoxy composites. In their work, multi-walled carbon nanotubes are dispersed into epoxy and infused into a glass fiber preform to form a conductive percolating network only at a very low CNT concentration [11]. The nanotube network throughout the whole composite polymer matrix system showed great sensitivity to initial variations of subtle matrix-dominated failure. Their dispersed CNT sensing networks are incorporated noninvasively into the composites and are capable of evaluating the onset and evolution of damage in composite structures. Combined with the in-situ electrical data monitoring, the integrated CNT sensing can be readily used to track the real-time micro-cracking formation 
and propagation and identify the nature and progression of composite damage globally $[12,50]$.

\subsection{Pristine CNTs Assemblies}

\subsubsection{CNT Yarns Strain Sensor - One-dimensional Assembly}

With the established drawable property of the CNT arrays grown by modified CVD method, nanotubes can be directly spun into yarns (shown in Figure 2.9(a)) by applying some twist to aligned CNT sheet pulled out from vertically aligned arrays. CNT yarns greatly absorbed the advantage of individual CNT being strong, stiff, light, small, corrosion resistive, electrical and thermal conductive. Based on these properties provided, they can be embedded into composite structure without deteriorating the structure integrity and thus functioning as effective strain and damage sensors [13].

By incorporating CNT yarns into composite structure, both Zhao [13] and Abot [3] used them as sensors to monitor strains and detect damage by different means. Zhao et al. placed CNT yarns on patterned sandpapers for aluminum deposition at two ends of yarn sensors functioning as electrodes (Figure 2.9(b)). Multiple yarns were then infused with epoxy resin and cured to form polymeric composites for real time structural health monitoring. Using a different fabrication method, Abot et al. [3] wrapped CNT yarns into a needle and then transversely stitched them throughout the central plies of the composite laminate, as shown in Figure 2.9(c). Zhao showed only a gauge factor of pure yarns at $\sim 0.5$ for both monotonic and cyclic tensile testing. However, a systematic aggregation of multiple CNT yarns is able to 
effectively monitor the propagation of cracks in the composite structure by observing the sudden resistance increases due to crack propagating across the yarns.
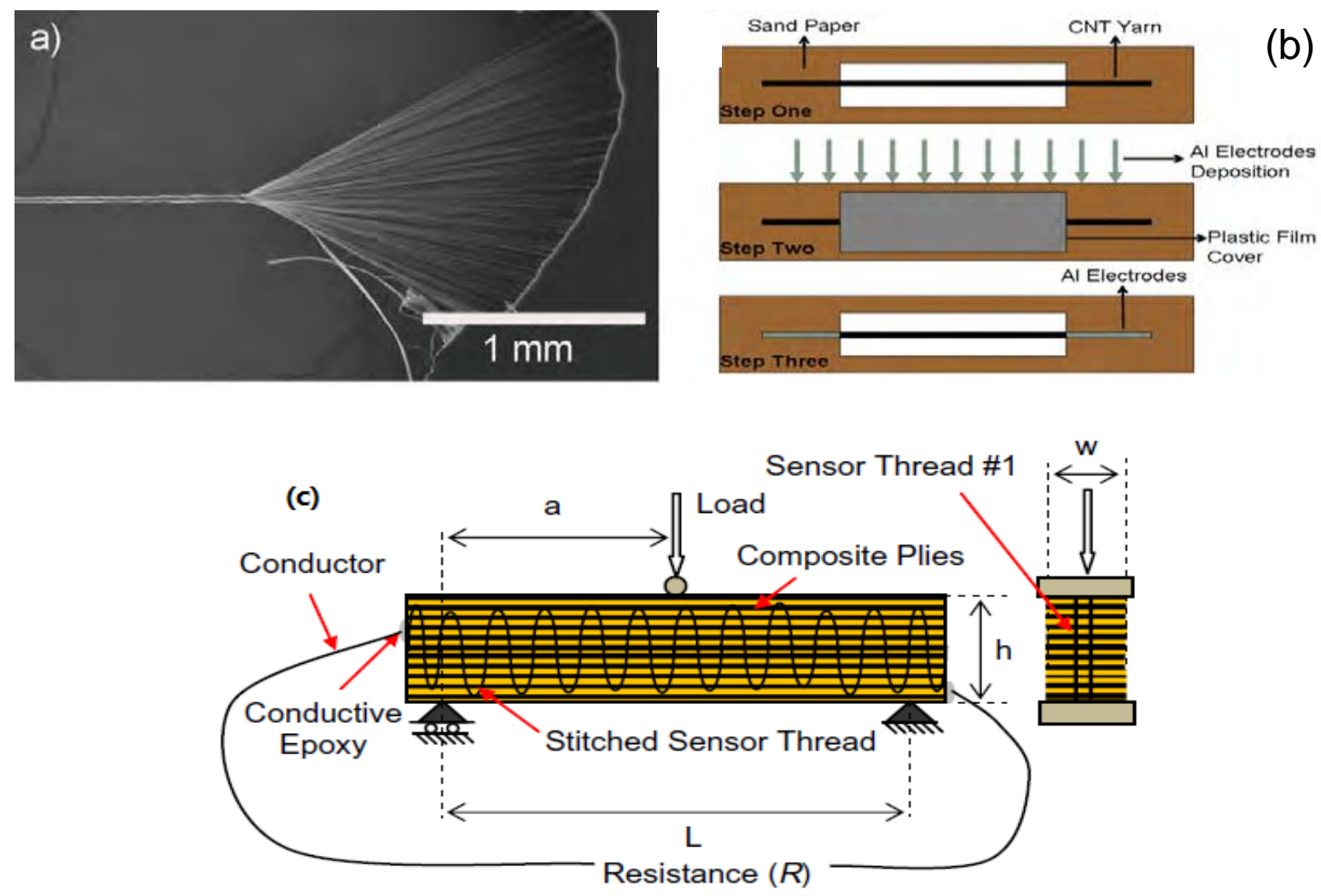

Figure 2.9 (a) SEM image of spinning CNT yarns from the as-grown CNT forest. (b) Process of Al electrodes deposition on a CNT yarn using magetron sputtering [13]. (c) Schematic of the side and cross-section of the laminated composite sample with transversely stitched CNT yarns under simultaneous three-point mechanical and twopoint electrical measurements [3].

\subsubsection{CNT Film or Bucky Paper - Two-dimensional Assembly}

P. Dharap et al. were among the most earliest to study the piezoresistive behavior of pure random oriented CNT film (bucky paper) [15]. The SWCNTs/dimethylformamide (DMF) 
dispersion was filtered through a $0.2 \mathrm{~mm}$ Teflon membrane to form buckypaper, which was peeled off from the filter and then attached onto a brass specimen for strain testing (Figure 2.10(a)). The buckypaper had isotropic electrical properties due to the random orientation of nanotubes and complex entanglement between nanotubes [97]. During the cyclic tensile and compressive testing, the voltage changes across the specimens were measured with a fourprobe method in situ. A relative linear and sensitive piezoresistive behavior was obtained when the bucky paper sensors were subjected to both tensile and compressive load shown in Figure 2.10(b). Nanotubes were deposited from the suspension DMF solution to bridge the electrodes in the work of Maune et al other than using the conventional filtration method [98]. The sensors made exhibited a linear piezoresistive behavior but with only a fairly low gauge factor of $\sim 1$.

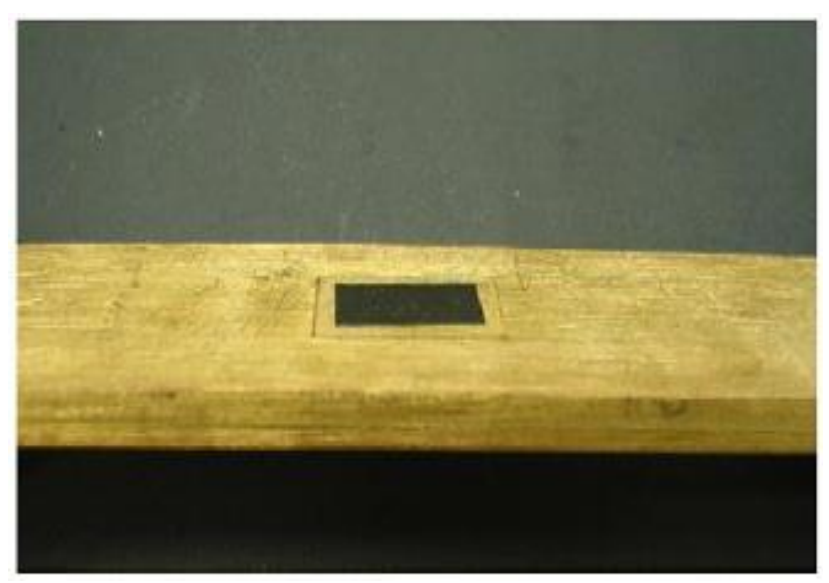

(a)

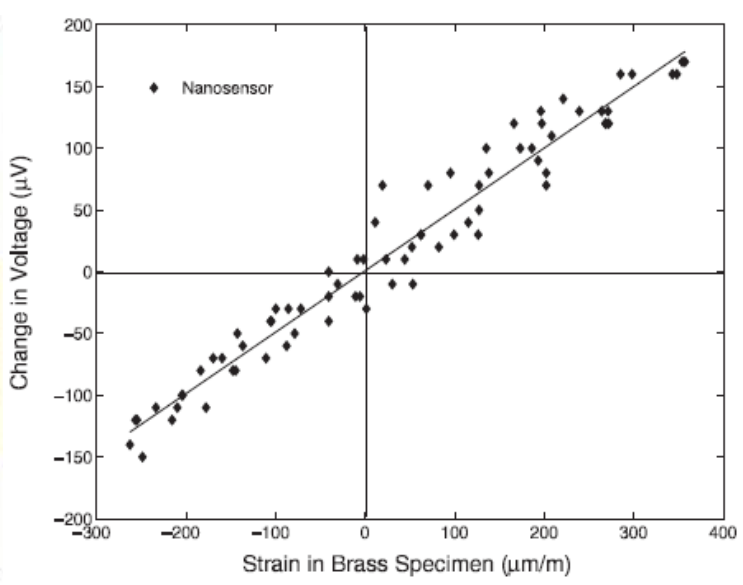

(b)

Figure 2.10 (a) SWCNT film with insulating PVC film attached to the brass specimen. (b) Voltage changes as a function of strain in the brass specimen [15]. 
Although some of the cited works in this section studied and concluded some factors influencing the sensing performance, the mechanism of macro-scale CNT based strain sensor is still in debate. The sensitivity of the piezoresistive behavior of different categories of sensors made with various manufacturing methods differs a lot in a range of $0.18 \sim 30.1$ (gauge factor). The way that the piezoresistive performance behave also differed from sample to sample (linear, non-linear, combined linear and non-linear). However, after gaining insight into the operating principles and the pros and cons of different fabrication methods and sensor morphologies, we feel that the combination of pure CNTs sheet with fiberglass/epoxy prepregs studied in this work is expected to be favorable for strain sensing in the areas of sensitivity, stability and repeatability. Our research covered some basic testing and data analysis to understand some fundamental knowledge on working mechanisms and performances, so as to lay a foundation for further development in the future. 


\section{Experimental Procedure}

The objective of this research is to fabricate a high performance carbon nanotube based piezoresistive strain sensors utilizing easy-to-use processing techniques. High mechanical flexibility and strain sensitivity are expected. The fabrication processing was simplified to make it both time-effective and cost-effective; reducing the laboring and energy cost and thus optimizing the overall manufacturing input. In the literature, common manufacturing methods require many steps and thus more complex and time consuming. To improve the quality of the nanotubes, surface modification and functionalization were usually applied to the raw CNTs by using surfactants or acid treatment. For the non-spinnable CNTs, good dispersion in the polymer matrix was very crucial because the nanotubes tend to aggregate together in the solvent forming defects. Typical methods of high shear mixing process and ultra-sonication were widely used, achieving good nanotube dispersion within the polymer system. In addition, degassing process was also needed to get rid of the air bubbles and avoid the intrinsic defects. After the mixing process, the mixture of CNTs and polymers can then be casted to the mold and cured at a proper temperature. Frankly speaking, many processes need to be conducted and controlled carefully in order to make high quality CNT composite sensors through existing methods. Whereas, thanks to the intrinsic uniform distribution of nanotubes in the aligned CNT sheet, extra physical or chemical treatment and mechanical dispersion process are not necessary, saving us much time and money. And the assembling of the SSP is very simple requiring little skill and laboring. Thus, overall speaking, the whole manufacturing process is optimized and very practical even for large-scale productions. In 
this research, the target specimens are fiberglass composite coupons with CNT SSP closely adhered to the surface of a testing gauge section.

\subsection{Materials}

\subsubsection{Multi-wall Carbon Nanotube (MWCNT) Sheet}

Spinnable MWCNTs arrays were synthesized through a chloride-assisted low pressure chemical vapor deposition (CVD) method, which consisted of individual CNT with 25-40 nm diameter and $1 \mathrm{~mm}$ length [76]. In the growth run, Iron (II) Chloride $\left(\mathrm{FeCl}_{2}\right)$ powder was used as catalyst placed in a ceramic boat under quartz substrate in quartz tube in the furnace. The furnace chamber was pumped to less than 10 mTorr, and then heated to $760{ }^{\circ} \mathrm{C}$. When the reaction temperature was reached, the growth gases were then released into the chamber with a composition of $600 \mathrm{sccm}$ acetylene, $395 \mathrm{sccm}$ argon and $5 \mathrm{sccm}$ chlorine. After 10 min of reaction, the gases were turned off and the system was purged with argon as it cooled to room temperature. The array purity was typically as high as $\sim 99.5 \%$ with very little residual iron catalyst left [76]. With the experimental parameters being properly controlled, our CNTs can be grown and drawn off in continuous sheets exhibiting good alignment of CNTs in macro-scale, as shown in Figure 3.1. On the other hand, the nanotubes in the CNTs sheet possess high aspect ratio (length to diameter ratio in the range of $25000 \sim 40000$ ), which is preferential to achieve larger area of inter-nanotube contact and thus electrical conductivity. 

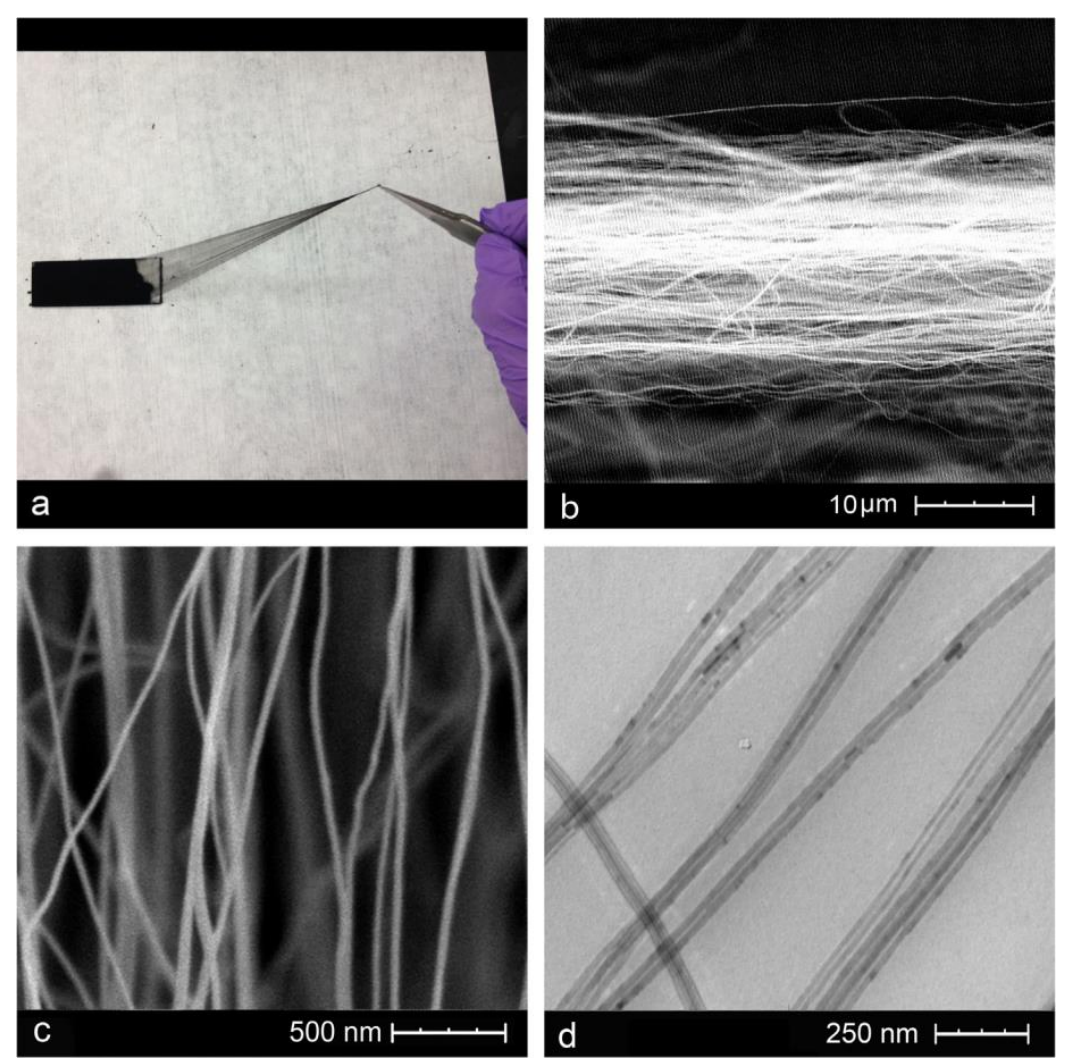

Figure 3.1 (a) CNT sheet being drawn from the array using tweezers, (b) SEM image of the side view of the CNT sheet showing the approximate thickness and low density structure, (c) high magnification SEM image of the top of the CNT sheet showing the spacing between CNTs and (d) TEM image of CNTs from the sheet [76].

\subsubsection{Fiberglass/Epoxy Prepregs}

Fiberglass/epoxy prepreg (Fibre Glast 7781 E-Glass prepreg) was used to fabricate the CNT strain sensing patches. The prepreg fabrics are pre-impregnated with an epoxy resin system. As a result, appropriate resin content and maximum, repeatable properties were attained. The data sheet for the pure resin gave a tensile modulus of $3.03 \mathrm{GPa}$ and elongation at break of 4.0\% [99]. Prepregs were chosen as the base material because when they are cured they have similar mechanical properties to the base composite, have consistent resin content, are easy 
to handle, provide structural stability to an otherwise thin and fragile CNT sheet and have a sticky surface which can be used to adhere the aligned CNT sheets during processing.

\subsubsection{Fiberglass Composite Substrate}

Pre-made fiber glass composites (G10/FR4 fiberglass laminate from American Epoxy and Metal Inc.) were used as substrates onto which the CNT SSPs were adhered. The bulk composite laminates were cut into coupons with a dimension of $300 \mathrm{~mm} \times 30 \mathrm{~mm} \times 6.35$ $\mathrm{mm}(\mathrm{L} \times \mathrm{W} \times \mathrm{T})$. The coupons were tabbed at the ends with $2 \mathrm{~mm}$ thick G10/FR4 fiberglass sheets with a taper angle of $20^{\circ}$. The sample and tab dimensions were selected so as to satisfy ASTM standard D3039/D3039M - 08 - Tensile Properties of Polymer Matrix Composite Materials [100].

\subsection{SSP Fabrication}

A very convenient fabrication processes is introduced in our research, which is time and cost effective and conductive to large scale sensor integration. First, the aligned CNT ribbons were directly drawn out from the CNT forest and adhered onto the prepreg, precut to dimensions of $50.8 \mathrm{~mm} \times 19.05 \mathrm{~mm}(2$ inches $\times 0.75$ inches $)$. Fine copper foils were cut into uniform dimensions $(20 \mathrm{~mm} \times 10 \mathrm{~mm})$ and used to fabricate the electrodes at the ends of CNT SSP. Next, the prepregs coated with aligned CNT sheet were placed on the center of the gauge section of tensile testing coupons with the CNTs side facing the composite coupon. A nylon peel ply and then breather cloth was placed on the outside of the SSP. The assembly was then vacuum bagged and the whole sample was finally cured at $154{ }^{\circ} \mathrm{C}$ for $\sim 1 \mathrm{~h}$ in a platen hot-press with no extra applied pressure. Since the CNT SSP was already closely 
integrated to the composite substrate, two wires of the same length $(\sim 1 \mathrm{~m})$ were soldered onto the surface of the two copper foil electrodes to achieve good electrical conductivity between the sensor and the measurement device. In order to get real-time and accurate strain measurement for reliable comparisons with the CNT SSPs, external commercial metal foil strain gauges (Vishay Micro-Measurements 250LW) were mounted on the opposite surface of the composite substrate (Figure 3.3b). The rough surfaces of our CNT patches might probably weaken the contact and adhesion of metal foil strain gauges, influencing the strain sensing accuracy. While the smooth surface of substrate coupon would favor the mounting of strain gauge and provide more accurate strain data from the surface. In addition, mounting the strain gauges to both sides would not make much difference for simulating the real-time strain variations of CNT SSPs. Therefore, we chose to mount the metal foil strain gauges on the composite substrate coupons. Samples were produced in which the CNTs were oriented in the direction of coupon loading (designated parallel samples) and where the CNTs were oriented $90^{\circ}$ to the direction of coupon loading (designated perpendicular samples). These fabrication steps are shown in Figure 3.2. We manufactured 20 sensors in total. The electrical resistance differed from each other due to the variation of hard-to-control CNT sheet morphology since we only use one single layer of nanotube sheet. But we tried to control the performance variations between specimens and minimize manual errors by regulating the fabrication processes. As measured in this work, the electrical resistances of as-fabricated sensors were controlled in the range of $800 \sim 1000 \Omega$ and $50 \sim 60 \mathrm{k} \Omega$ for parallel and perpendicular SSPs, respectively. 


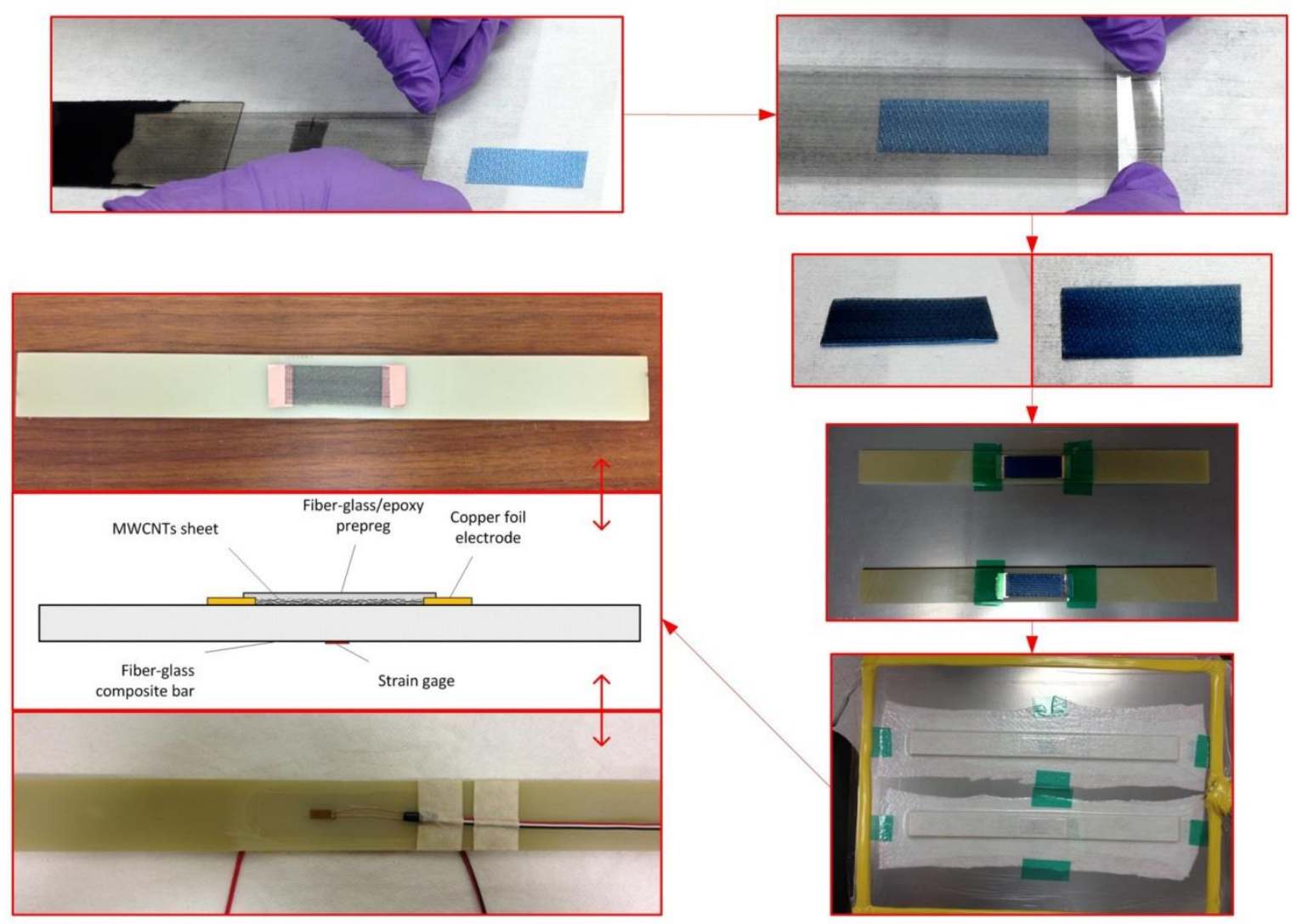

Figure 3.2 Step by step fabrication process and schematic configuration of testing specimens.

\subsection{Testing System}

In order to evaluate the sensing performance of the CNT SSP, different types of experiments were performed using a MTS Landmark servo hydraulic $250 \mathrm{kN}$ test systems, including: (1) monotonic tensile tests, (2) constant strain rate cyclic tensile tests, (3) 1000 cycle fatigue tests and (4) load controlled incremental loading-unloading tensile tests. The typical experimental set-up of the mechanical testing equipment is shown in Figure 3.3. 


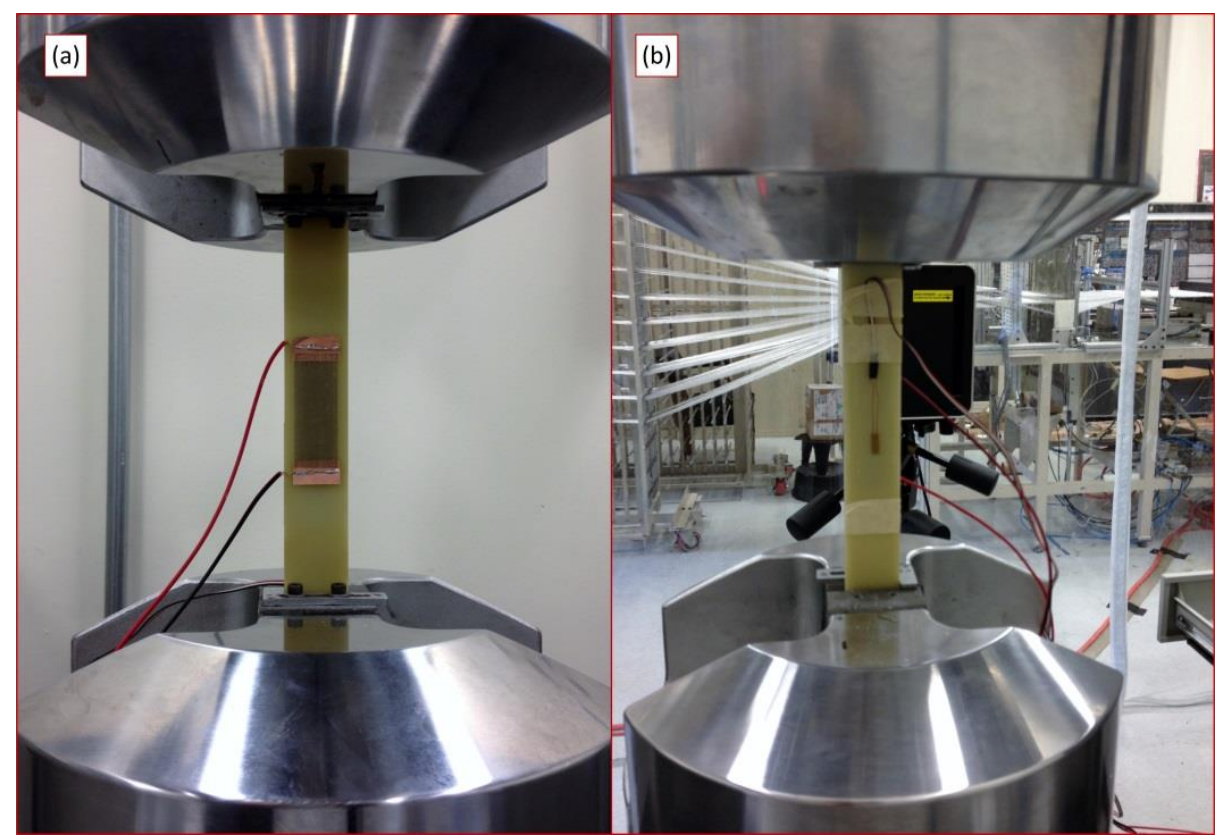

Figure 3.3 Typical mechanical tensile test set-up. (a) front surface with a CNT SSP, (b) back surface with a metal foil strain gauge.

The external commercial metal foil strain gauges were utilized on the opposite side of the composite to provide accurate real-time strain data. In all types of tensile tests, the composite coupon was subjected to mechanical loading, while the electrical resistance of the CNT SSP was recorded simultaneously by an Agilent 34420A multimeter at a frequency of $10 \mathrm{~Hz}$. The resistance measurements were conducted by using four-probe method along the longitudinal direction of the composite coupon substrate to minimize the contribution of wires and connections to contact resistance. Generally, the following data were all simultaneously recorded: (1) force and displacement from the testing machine, (2) strain from the external strain gauge and (3) electrical resistance from the multimeter. The mechanical-electrical test set-up and data acquisitions are presented schematically in Figure 3.4. 


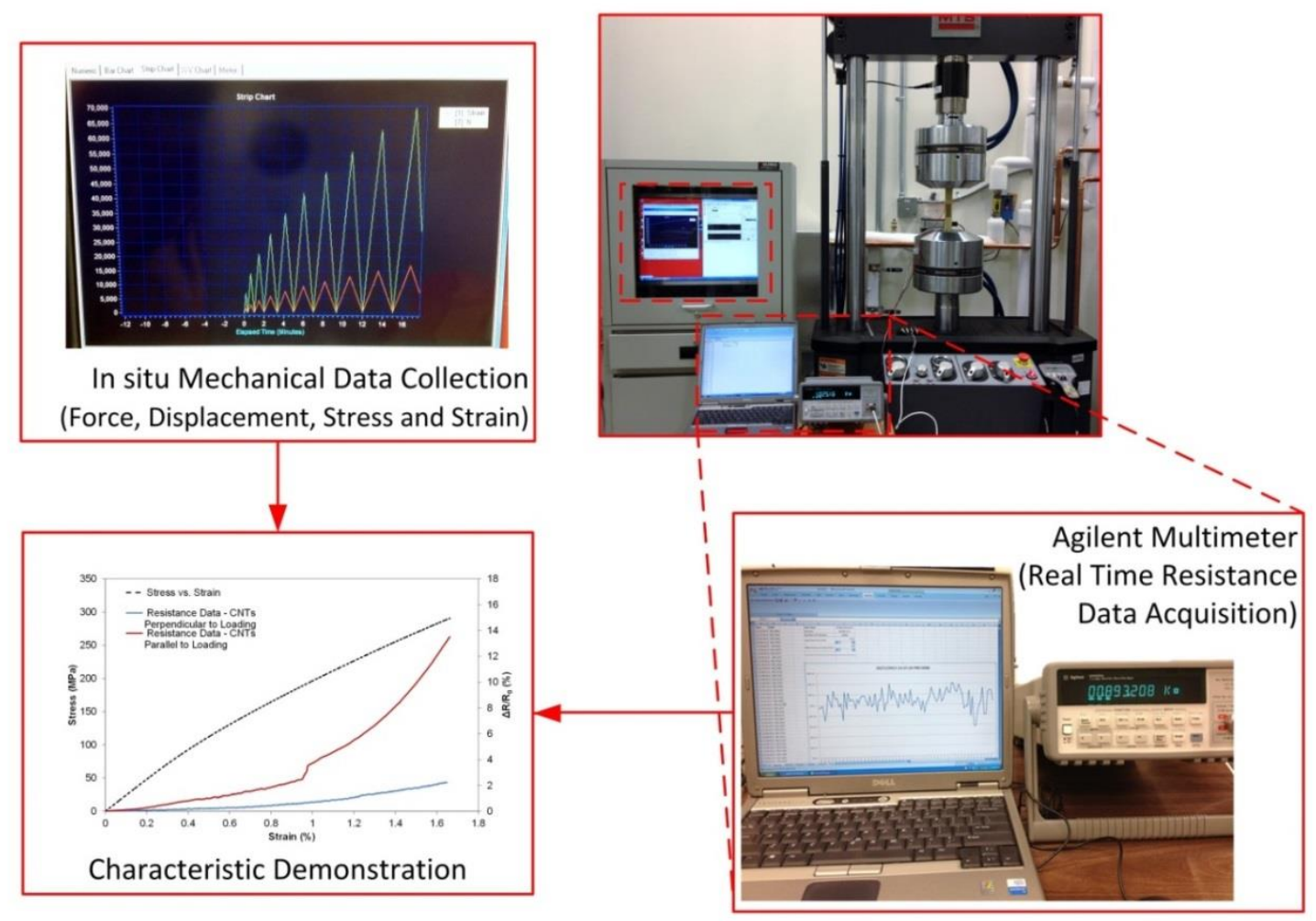

Figure 3.4 Coupled mechanical-electrical test and data acquisition system. The electrical data was collected in-situ while the mechanical tensile tests were ongoing.

Monotonic tensile tests were performed with a fixed displacement speed of $1.5 \mathrm{~mm} / \mathrm{min}$. In total, 6 different specimens (3 parallel and 3 perpendicular specimens) were monotonically strained to failure. For the case of the incremental tensile tests, the specimens were loaded and unloaded at the same rate with progressively increasing peak values of cyclic loading with a step value of $7 \mathrm{kN}$ until coupon failure. A total of 6 different specimens (3 parallel and 3 perpendicular specimens) were tested in the defined progressive damage accumulation loading - unloading cycles. At the end of each cycle, the specimens were unloaded to zero 
loading state of the machine, in order to monitor the induced damage via residual strain measurements of the coupon and corresponding irreversible resistance change of SSPs. All the mechanical tests were conducted in a controlled laboratory environment. The temperature vs. resistance behavior was investigated while the specimen was kept in a Ransco RTH-200$\mathrm{S}$ thermal chamber. 


\section{Experimental results and discussion}

\subsection{Stability Evaluation of CNT SSPs}

SHM requires sensing under severe environmental conditions (Temperature \& Humidity) for very long time. The stability of strain sensors played a significant role during the serving cycles. Before evaluating the endurance of strain sensors to various environments, we need to first have some basic knowledge of the intrinsic electrical resistance stability of our CNT SSPs. Further studies with respect to the sensing stability would be investigated on this basis in the future.

The electrical resistance behavior of a CNT SSP measured over multiple days, under room temperature, ambient atmosphere and no-load condition, is shown in Figure 4.1(a). It can be seen that the electrical resistance readings fluctuate in very small amplitude (within $\sim 0.1 \%$ of the initial reading over $120 \mathrm{~h}, \mathrm{CV} \%$ of $0.031 \%$ as calculated), meaning that the sensor displayed excellent stability in the aspect of long-term electrical resistance measurement showing no significant change all along. This is probably due to the intrinsic physical

structure of the CNT sheet in which the millimeter long CNTs have a large amount of contacts with their neighbors and thus high conductivity and provide a low overall sensor resistance. Some CNT/polymer composites suffer from electrical resistance drifting over time with no applied mechanical load [35] which may limit their capability to function as sensing materials in practical applications. The problem can be traced to the defects in CNTs wall structures and the localized heating caused by electrical current concentration [35]. In comparison, large amount of nanotube contacts can effectively eliminate the undesired 
heating effect by building up adequate and effective electrical pathways and thus give CNT SSP better long term electrical resistance stability. While it was not tested, it is expected that the composite nature of the CNT SSP which contains an epoxy resin system, would also protect the CNTs from moisture and chemical corrosion.

(a)

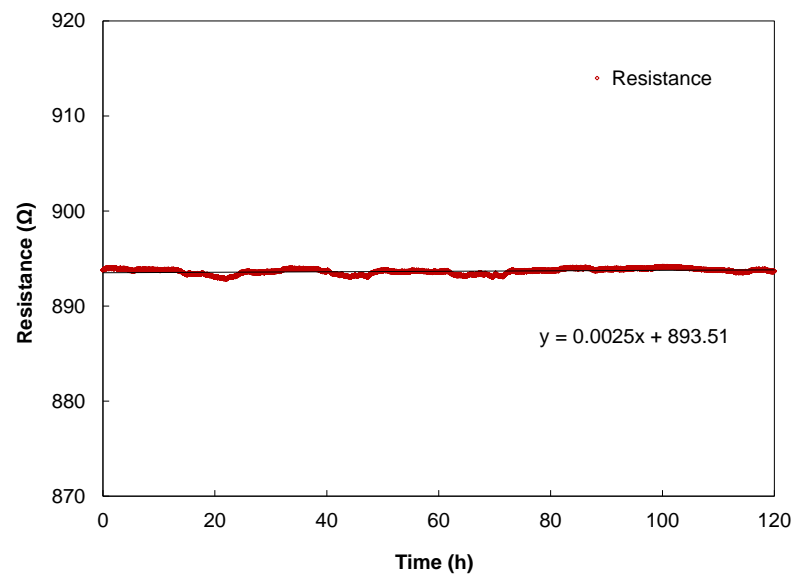

(b)

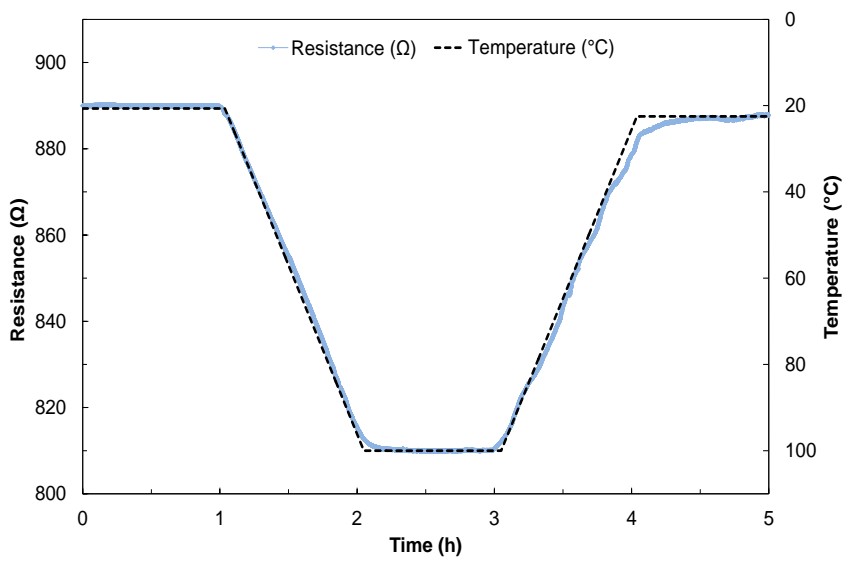

Figure 4.1 (a) Long term intrinsic resistance stability: the resistance of the SSP was measured with zero load applied at room temperature over 120 hours. (b) Electrical resistance reacted correspondingly to the temperature variation in the range of $22 \sim 100{ }^{\circ} \mathrm{C}$ and was able to keep the value at relatively high temperature over time. 
The electrical resistance of CNT SSP showed good stability during $5 \mathrm{~h}$ dynamic thermal test under a zero mechanical load condition, as shown in Figure 4.1(b). The sensor was kept in the thermal chamber and the instant electrical resistance was measured while the sensor was under a predesigned heating and cooling program. First, the sample was kept at room temperature for $1 \mathrm{~h}$. Then the chamber temperature was increased uniformly from room temperature to $100 \mathrm{C}^{\circ}$ (approximate max use temperature for the resin system) in $1 \mathrm{~h}$ and allowed to soak at $100 \mathrm{C}^{\circ}$ for another $1 \mathrm{~h}$. Finally, the sample was cooled down to original temperature in $\sim 1 \mathrm{~h}$ and held at that temperature for one more hour. It was observed that the electrical resistance of the CNT SSP remained stable during the soaking time at both room and high temperature, indicating its promise to be applied in a relatively large temperature range. It is in a good agreement with previous experimental results of highly conductive CNT sensors, e.g. H Zhao et al. also reported the good stability of CNTs yarn sensors during 100 min of measurements at $-196,25$ and $110{ }^{\circ} \mathrm{C}$ [13]. More importantly, the electrical resistance increased reversibly during cooling stage. It is shown from the figure that the curves of the temperature profile and electrical resistance profile match well with each other displaying a good linear correlation between the electrical resistance responses toward temperature change. Similarly, a linear thermal piezoresistive behavior was also observed for CVD grown pure MWCNT forest [101], MWCNT sheets [102] and aligned MWCNT papers [103]. However, the electrical resistance for pure CNTs assemblies was normally found to decrease exponentially with the increase of the temperature due to variable range hopping and 
tunneling conduction mechanism [104]. This unique linear thermal-electrical property is still not clearly understood yet.

For the case of our SSP specimens, it is speculated that the epoxy matrix might affect the overall thermal-electrical behavior. M.K. Njuguna et al. claimed in their study that CNTs nanocomposite sensors are very sensitive to environmental temperature due to the thermal behavior of polymer matrix. In that work they observed up to $140 \%$ resistivity increase in response to a $70 \mathrm{C}^{\circ}$ temperature increase [38]. As the thermal expansion coefficient of polymer is much larger than that of CNTs, when temperature increases, the inter-tubes distance in the CNT network is increased, resulting in increase of electrical resistance. This thermal expansion effect would be particularly obvious at higher temperature. Thus, the increase of the resistance due to the thermal expansion was possibly compensation to the exponential electrical resistance decay controlled by the intrinsic semi-conductive property of CNTs. The electrical resistance change relevant to the temperature variation in this study is thus speculated as due to a combination of thermal expansion effect of polymer matrix and intrinsic electrical properties of the CNTs grown in our lab. Although there is a little electrical resistance deviation and hysteresis at the end, the sample tested here showed an overall consistent and reversible electrical resistance/temperature behavior which is beneficial to function as a thermostable sensor over wide range of temperature and operating conditions. Koratkar et al. also indicate that, if well-tailored, vertically aligned CVD grown MWCNTs are promising to serve as extremely light-weight, ultra-thin, minimally-intrusive 
temperature sensors that are robust, sensitive, reliable and repeatable [101]. Our results show that thin sheets of aligned CNTs in strain sensing patches can serve the same purpose.

\subsection{Sensitivity and Linearity Behavior}

\subsubsection{Monotonic Quasi-static Tensile Test}

In order to investigate the piezoresistive response of the SSP, the electrical resistance of the sensors was measured in situ while the sensors were mechanically strained in the axial direction of the coupon. Typically, the initial electrical resistance would be measured for certain period of time to stabilize the sensors before the mechanical loading. Due to the superior electrical conductivity along the CNT longitudinal direction, the parallel SSPs have much lower electrical resistance $(800 \sim 1000 \Omega)$ values than those of perpendicular specimens $(50 \sim 60 \mathrm{k} \Omega)$ as measured in our work. The typical mechanical tensile stress $(\sigma)$ vs. strain $(\varepsilon)$ curve and normalized resistance change $\left(\Delta R / R_{0}, \Delta R-\right.$ the real-time resistance minus original resistance, $\mathrm{R}_{0}$ - original resistance before straining) of the SSPs as a function of strain are plotted altogether in Figure 4.2. 


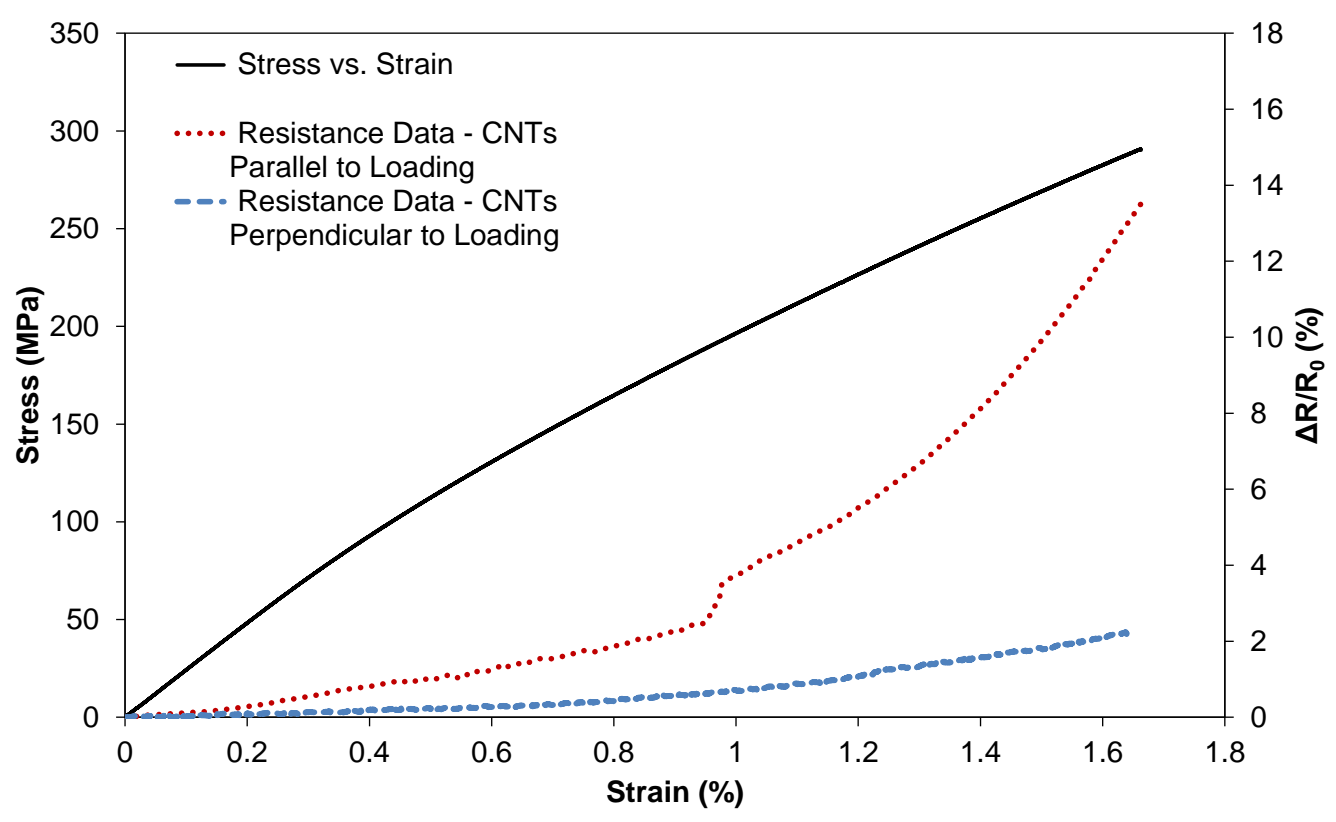

Figure 4.2 The mechanical stress/strain curve of monotonic tensile test and normalized resistance change $\left(\Delta R / R_{0}\right)$ of parallel and perpendicular SSPs versus strain $(\varepsilon)$. The $\Delta R / R_{0}$ increases linearly at lower $\varepsilon$, then follows a non-linear behavior thereafter. The response of $\Delta R / R_{0}$ to $\varepsilon$ for the parallel SSP is more sensitive.

Two types of specimens with different CNTs orientations (parallel and perpendicular to the loading direction) were tested to understand the influence of orientation of CNT on the piezoresistivity of the SSP. The parallel SSP showed relatively good linear response of electrical resistance change to strain at lower level $(0 \sim 0.8 \%)$. Then, an abrupt change in $\Delta \mathrm{R} / \mathrm{R}_{0}$ was observed around the strain of $\sim 0.95 \%$ in all the parallel specimens tested, which might be due to the micro-cracking within the SSP intrinsic structure. Following the steep resistance variation, the $\Delta R / R_{0}$ increases in a faster manner than that in the low strain regime, revealing a non-linear piezoresistive behavior (exponentially or polynomially as predicted). 
The perpendicular sensors showed more linear piezoresistive trend overall, yet a change in curve slope can also be detected in the higher strain range, indicating an internal structure change of the SSP as well.

For strain sensing, a high sensitivity to applied strain is desirable in practical applications. The sensitivity is generally estimated by Gauge Factor (GF) in Eq. (1), which relates the normalized resistance change $\left(\Delta \mathrm{R} / \mathrm{R}_{0}\right)$ to the applied mechanical strain $(\varepsilon)$.

$\mathbf{G F}=\left(\Delta R / R_{0}\right) / \varepsilon$

The GF is the slope of the coupled $\Delta \mathrm{R} / \mathrm{R}_{0}$ and $\varepsilon$ curve. As shown in Figure 4.2 the parallel SSP has much higher GF which will be more beneficial in the aspect of strain sensing. Considering that the gauge factor of conventional alloy metal foil stain gauges is around 2, the CNT SSP is fairly competitive in this regards.

To explain the experimental results, we need to refer to the working mechanisms of the CNTs nanocomposite strain sensors in the literature. As described in section 2.3 of literature review, much research has been done on CNTs dispersed nanocomposites with various types of polymer matrix. However, our SSPs with aligned CNTs sheet are fairly unique structure for piezoresistive strain sensing. The CNTs sheet is consist of hundreds of thousands of aligned long nanotubes with some waviness, forming a network of dispersed bundle-bundle connections or contacts. So the CNTs inside the matrix are overlapping or entangled at the contact locations, rather than being arranged in an end-to-end configuration $[39,104]$. 
From the accumulated knowledge to date, the piezoresistive behavior of CNT SSP can be mainly attributed to three aspects: (1) the intrinsic piezoresistivity of individual CNTs due to the energy band change in respond to external strain (2) variations of contacting internanotube electrical resistance, e.g., decrease of CNTs contact area or (3) tunneling resistance change between neighboring CNTs due to inter-distance change caused by cracking or relative motions $[31,54,96]$. The redundant electrical conductive pathways from the bundled CNTs ribbon structure give the specimens fairly high conductivity and thus much smaller resistivity than those CNTs dispersed polymer nanocomposites. So the contact resistance initially dominates the total resistance and the tunneling resistance contributed as the minor part. With the further straining of the nanotube network, the tunneling resistance might gradually dominate the total piezoresistive resistance increasing the sensitivity. The mechanisms of parallel and perpendicular SSPs are partly different and thus will be discussed separately.

For the parallel SSPs, at the first stage of straining $(0 \sim 0.8 \% \varepsilon)$, the specimens passed through a pure elastic deformation zone, where a quasi-linear piezoresistive curve can be obtained, yet with smaller gauge factor compared to that in the higher level strain range. With the increase of strain value, the formation of nano-cracks was most likely initiated in the epoxy matrix system. The introduction of CNTs would inevitably cause non-uniform stress distribution throughout the matrix area, which often leads to the onset of cracking at relatively low stress or strain level [54]. Due to the extremely large aspect ratio of CNTs, load was assumed to be transferred well along most part of the nanotube length. While 
theshear stress concentrations were severe at the ends of individual CNTs, initial nanoscopic cracks are more likely to form in vicinity of these zones in the matrix. Particularly at the nanotube bundled and entangled areas, the nano-cracking would enable the relative motions of nanotubes against each other. This would thereby cause the decrease of CNTs contact area and thus increased inter-tube contact resistance when nanotubes slide against each other under straining. Thus, the quasi-linear increase of $\Delta \mathrm{R} / \mathrm{R}_{0}$ to $\varepsilon$ in this range can be attributed to the steady decrease of CNTs contact resistance from the gradual transverse crack propagations and accumulations [105]. Besides, sudden formations of cracks would uncontrollably influence the nanotube conductive network and cause noise effect creating fluctuations of electrical signal, which were present on the $\Delta R / R_{0}$ vs. $\varepsilon$ curve. In summary, for this particular regime, the sensing behavior of CNTs SSP is expected to be substantially affected by the variations of the physical spacing and direct contact among MWCNTs derived from matrix cracking initiation, propagation and accumulation. On the other hand, the load applied might be partially transferred from the matrix to the long nanotubes slightly straining the CNTs. Thus, the intrinsic piezoresistive property of individual CNTs might have some secondary contributions to the overall coupled electrical-mechanical behavior.

The jump in the shape of the normalized resistance change to strain curve was generally found in all tested parallel specimens around the strain ratio of $0.95 \%$. When the structure is further strained after the elastic deformation zone, the nanoscopic cracks in the matrix would likely to propagate and grow into micro-cracking in the transverse direction normal to the uniaxial loading which might be similar to the case in [11]. Due to the distinct stiffness of 
CNTs and epoxy and the transverse cracking which is normal to CNTs in parallel SSPs, further mechanical strain would be more likely to cause nanotube fracture and interrupt the nanotube electrical current pathways locally. As the CNTs electrical conductive networks would be damaged in some spots due to the random nanotube fracture, a sudden drastic resistance change would be triggered at a critical strain level, reflected exactly by the steep increase of $\Delta \mathrm{R} / \mathrm{R}_{0}$ around $0.95 \% \varepsilon$ on the piezoresistive curve. The increase of the electrical resistance would not fully recover even when the cracks were closed during the unloading procedure. This is proved later in the mechanical testing where incremental stress was applied progressively to the composite coupons. Irreversible strain was induced at higher loading level accompanied by permanent electrical resistance increase. As the bundled CNTs sheet structure is the same among SSPs, this phenomenon is commonly seen at certain critical strain value $(\sim 0.95 \% \varepsilon$ in our case). It is reasonable to deduce that the crack initiation would start in the epoxy matrix at low strain value, because CNT loaded epoxies often fail before their listed failure strain. Ogasawara et al. observed the failure strain in the range of $0.3 \sim 0.5 \%$ for aligned MWCNTs/epoxy composite in contrast to $3.8 \%$ for pure epoxy [106]. It was also explained by $\mathrm{Fu}$ et al. in their paper that the reduction in the failure strain is caused by an embrittlement effect as the stiffness of the composites is improved when loaded with very short fiber fillers [107].

In Figure 4.2, following the elastic deformation zone, starting right after the abrupt $\Delta R / R_{0}$ increase, the normalized electrical resistance change responded to the increase in the strain more rapidly. This accelerated electrical behavior can very likely be attributed to the 
reduction in $\mathrm{CNT}$ to $\mathrm{CNT}$ contacts and the tunneling effect. At lower strain range, the epoxy matrix nano- and micro- cracking merely formed around weak spots where there were severe stress concentrations. While, at higher strain values, the formerly created cracks could further expand and propagate along the interfaces of nanotubes and matrix. In this case, gaps would form in between the nanotubes and epoxy matrix, leading to the increase of nanotube to nanotube distance. Theoretically, if the inter-nanotube separations are small (in the order of nanometers), electrons would be able to hop across the gaps. So in this condition the tunneling currents would be responsible for the electrical conductivity. The constitutive equation which correlates normalized resistance change $\left(\Delta R / R_{0}\right)$ and strain $(\varepsilon)$ has originally been derived by Zhang et al. following the Simmons theory for tunneling resistance [108],

\section{$\Delta R / R_{0}=(1+\varepsilon) \exp \left(\gamma s_{0} \varepsilon\right)-1$}

Where $\varepsilon$ is the strain, $\mathrm{s}_{0}$ is the initial tunneling distance (unloaded state) and $\gamma$ is a factor that depends on the difference in work functions between the nanotube and the matrix [109].

Hu et al. found that the increase of either the tunneling resistance or the ratio of the tunneling resistance to the total value is the key factor to improve the sensitivity of CNTs-based sensors [95]. Hence in our case, with the increase of the curve slope under increasing strain, the contribution of the tunneling resistance increases as well and gradually dominates the overall resistance of the SSP. The variation of resistance change hereafter will follow a rule influenced more by tunneling effect, leading to a more rapid surge of $\Delta R / R_{0}$. However, because of the bundled structure of CNTs ribbons, the inter-tube contact electrical resistance would still influence the variation of overall $\Delta \mathrm{R} / \mathrm{R}_{0}$ to some extent. Thus, it is believed that 
the slope increase in this relatively high strain regime is due to a coupled effect of both a decrease in the number of $\mathrm{CNT}$ to $\mathrm{CNT}$ contacts and larger tunneling distance between some tubes.

For perpendicular SSPs, the orientation of CNTs is largely normal to the loading direction. The nanoscopic cracks at the early stage of straining would directly propagate transversely along the interface of nanotubes and epoxy matrix. In contrast to the situation of parallel SSPs, transverse cracks would not damage conductive pathways along the nanotubes as much, so the connections of CNTs were thus better preserved. In this condition, the bonding in between CNTs and matrix would be damaged at very low level of strain. However, due to the waviness of individual CNTs and the good inter-nanotube entanglement in the CNTs bundles, the conductive pathway in the direction normal to the nanotube orientation would not be interrupted significantly. As shown in Figure 4.2, the electrical resistance change was lower at low strain level giving a smaller gauge factor than parallel SSPs. With a higher level of strain applied to the CNTs network, some physical attachments between nanotubes would be destroyed due to the continuous expansions of nano-cracks in the matrix. So the electrons would hop across the gap in between the nanotubes and form tunneling current. In this case, when the SSP is further strained, the tunneling resistance would contribute more and gradually dominate the overall resistance of the SSP.

Thanks to the good alignment of CNTs ribbon at the loading direction, the parallel specimens have much higher sensitivity than perpendicular ones. The GF of typical parallel SSPs are 
largely in the range of $1 \sim 8$ while the perpendicular SSPs curve presented their GF from 0.5 to 1.3, as shown in Figure 4.3.

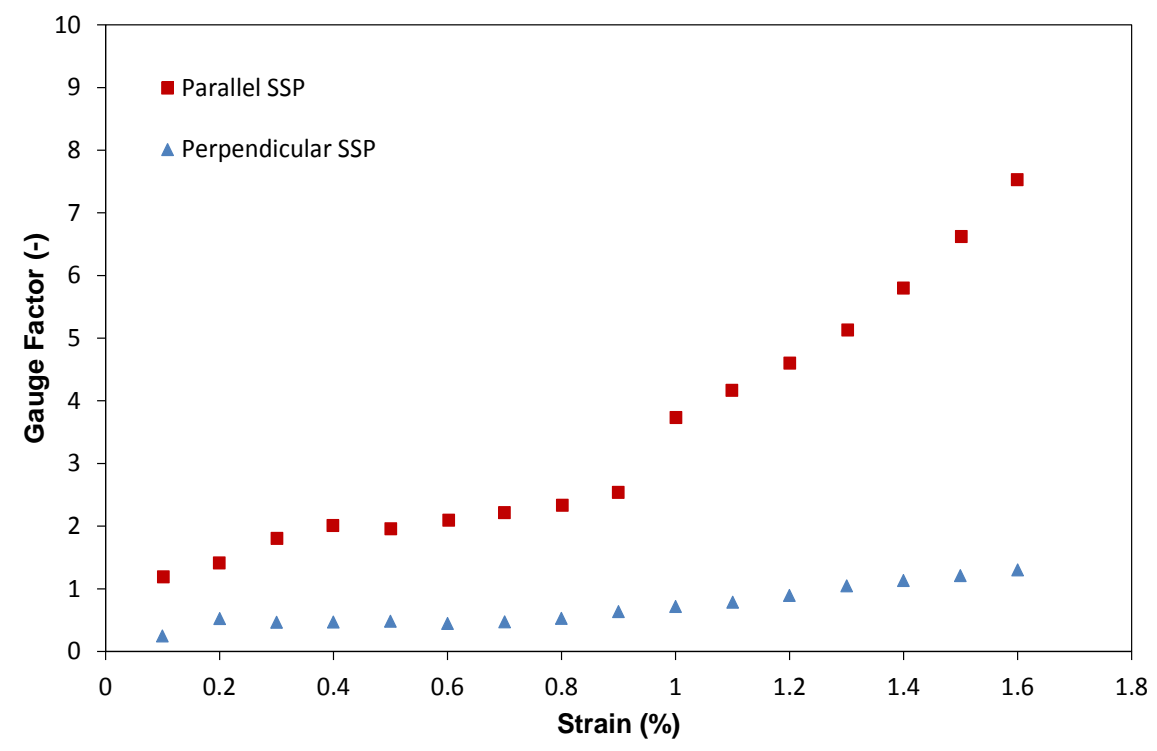

Figure 4.3 Gauge Factor of parallel and perpendicular SSPs at different strain levels in the monotonic tensile testing till fracture.

The good conductivity of well aligned CNTs sheet gives the sensor favorable piezoresistive property with little noise effect. For the perpendicular specimens, the low sensitivity of electrical response to strain limits its direct utilization for sensing applications in comparison with parallel SSPs. 


\subsubsection{Cyclic Uniaxial Tensile Test}

The quasi-static tensile tests have already proved good sensitivity and linearity within a certain strain range. Yet, the specimens may be permanently damaged by too much external mechanical force and not able to function properly again. Thus, cyclic tensile tests were needed to see whether good piezoresistive behavior can be maintained and to determine a proper functional strain range (upper strain limit) for our samples. Figure 4.4 shows the piezoresistive responses of the parallel and perpendicular CNT SSPs under cyclic loading and unloading. Both types of the sensors were stretched to the strain of $0.8 \%$ and then unloaded back to zero for 10 cycles in total. The composite coupons were cyclically loaded within its estimated elastic regime $(\varepsilon<0.8 \%)$ at a rate of $2 \mathrm{cpm}$.

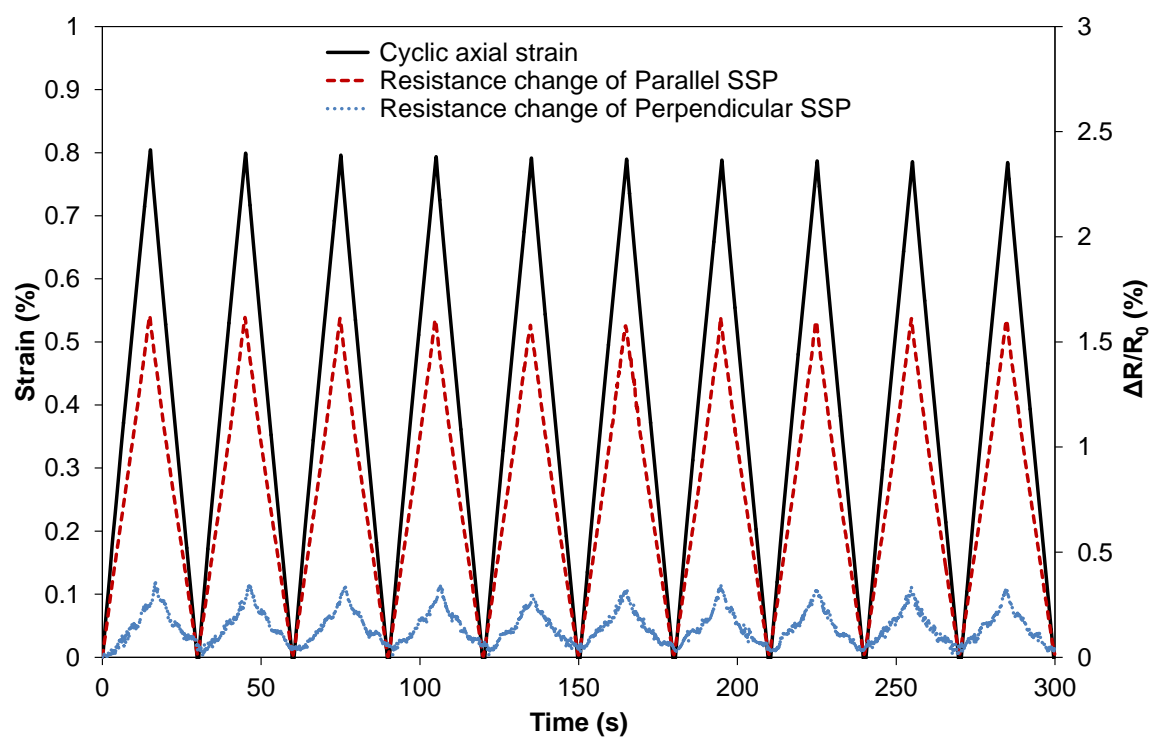

Figure 4.4 Constant strain cyclic tensile test: normalized electrical resistance change of parallel and perpendicular SSPs versus time in response to ten cycle loading unloading of $0.8 \%$ strain. 
The normalized electrical resistance change show very good linear behavior under cyclic strain and the linearity is maintained for multiple cycles without any significant resistance drifting. The curve of electrical resistance change data for the perpendicular sensor shows more severe noise effect during the cyclic tensile test. Comparing the CNT SSP with different nanotube orientations, the parallel sensors obviously showed much higher sensitivity to the strain, obtaining a gauge factor approximately four times that of perpendicular sensors. The SSP thus has a favored unidirectional sensing property along the nanotubes alignment direction, which was also observed in [13]. As indicated by the results in Figure 4.4 , in the proper strain range $(0 \sim 0.8 \%)$, the linear piezoresistivity with consistent sensitivity (GF value) can be preserved for both types of CNT SSPs after multiple cycles of loading and unloading, showing no obvious hysteresis over short time test. In this case, a reasonable working range $(0 \sim 0.8 \%)$ for strain sensing that is compatible with many types of high-performance advanced composite materials is thus proposed. For example, high strength fibers (e.g., glass fibers and carbon fibers) reinforced thermoset polymer composites exhibit high strength and stiffness along the direction of reinforcing fibers, becoming less ductile and even brittle. This would inevitably result in the relative small strain to failure value, in the range of $1 \sim 2 \%$. Normally, cracks and damage initiations can be detected well before the failure strain to prevent the complete fracture or breakage of the whole composite. Moreover, the distinct performances of SSP with orthogonal CNTs orientations can be utilized to not only detect the strain that the target composite structure is undergoing, but also largely 
identify the direction from which the external load is applied. This functionality is helpful to achieve more comprehensive and accurate SHM for bulk composite structures.

\subsection{Consistency and Damage Sensing Analysis}

\subsubsection{Progressive Damage Accumulation Test}

Progressive damage accumulation (PDA) tensile test is believed to be very suitable to estimate the potential of the nanocomposite systems for the sensing of stresses, strains and occurring damage [57]. The incremental tensile loading step was approximate $7 \mathrm{kN}$, inducing damage to the material which can be noticed as residual strain after each unloading step. The responses of parallel and perpendicular SSPs to the defined incremental mechanical loading unloading procedure can be seen in Figure 4.5. In this figure, the applied stress and strain as measured by the external strain gauge and the electrical resistance change of two types of SSPs are all plotted versus time, in order to analyze the corresponding piezoresistive behavior and also the damage detection capability for SSPs with both CNTs orientations. 


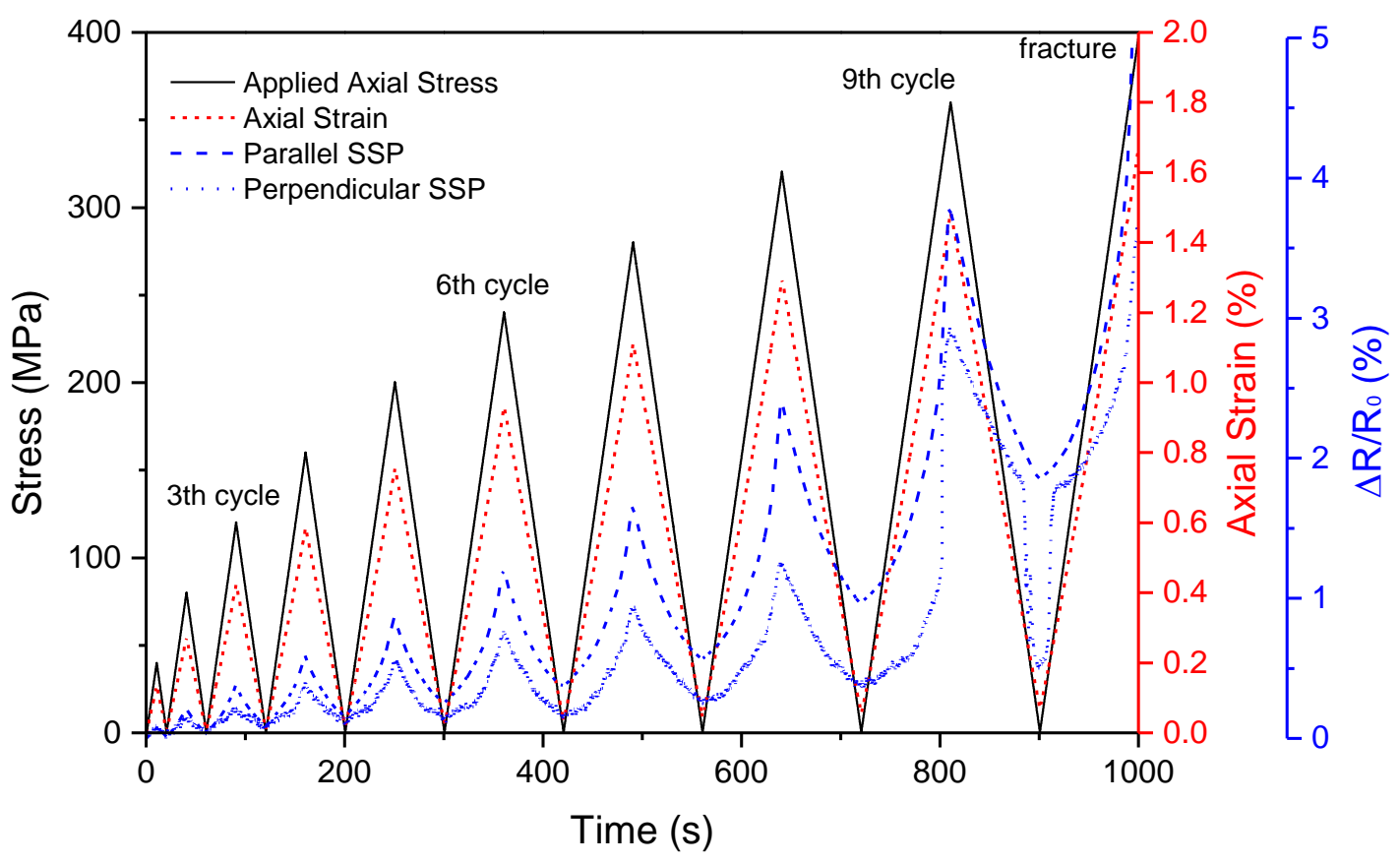

Figure 4.5 The real-time electrical resistance variation of parallel and perpendicular SSPs as well as stress/strain data corresponding to progressive damage loading with 7 kN increment for each cycle until fracture. The stress was unloaded to zero after each cycle, producing residual strain and irreversible $\Delta R / R_{0}$ of both parallel and perpendicular SSPs.

It is demonstrated in Figure 4.5 that after the fourth loading cycle some residual strain in the test coupon occurs when the specimen is unloaded to zero. The residual strain increases with each subsequent loading cycle and can be attributed to viscoelastic matrix deformation and damage occurring, e.g. transverse matrix micro-cracking [50,57]. It is clearly observed that the change in electrical resistance can be correlated with the applied cyclic loading unloading. The electrical resistance change increases and decreases monotonically with the incremental cyclic stress/strain protocol. A similar behavior was noticed for the case of electrical resistance change of SSPs with both CNTs orientations. Also after the fourth 
loading cycle, a distinct irreversible resistance change occurs when the specimens were unloaded. This directly correlates with the emerging residual strain which could be observed noticeably after the fourth loading cycle (Figure 4.5). This result also underlines the potential capability of SSPs for damage sensing applications. Figure 4.6 shows the correlation of the emerging residual strain in the composite coupon substrates after each cycle of loading unloading in PDA tests with the corresponding irreversible resistance change of CNT SSPs measured instantaneously.

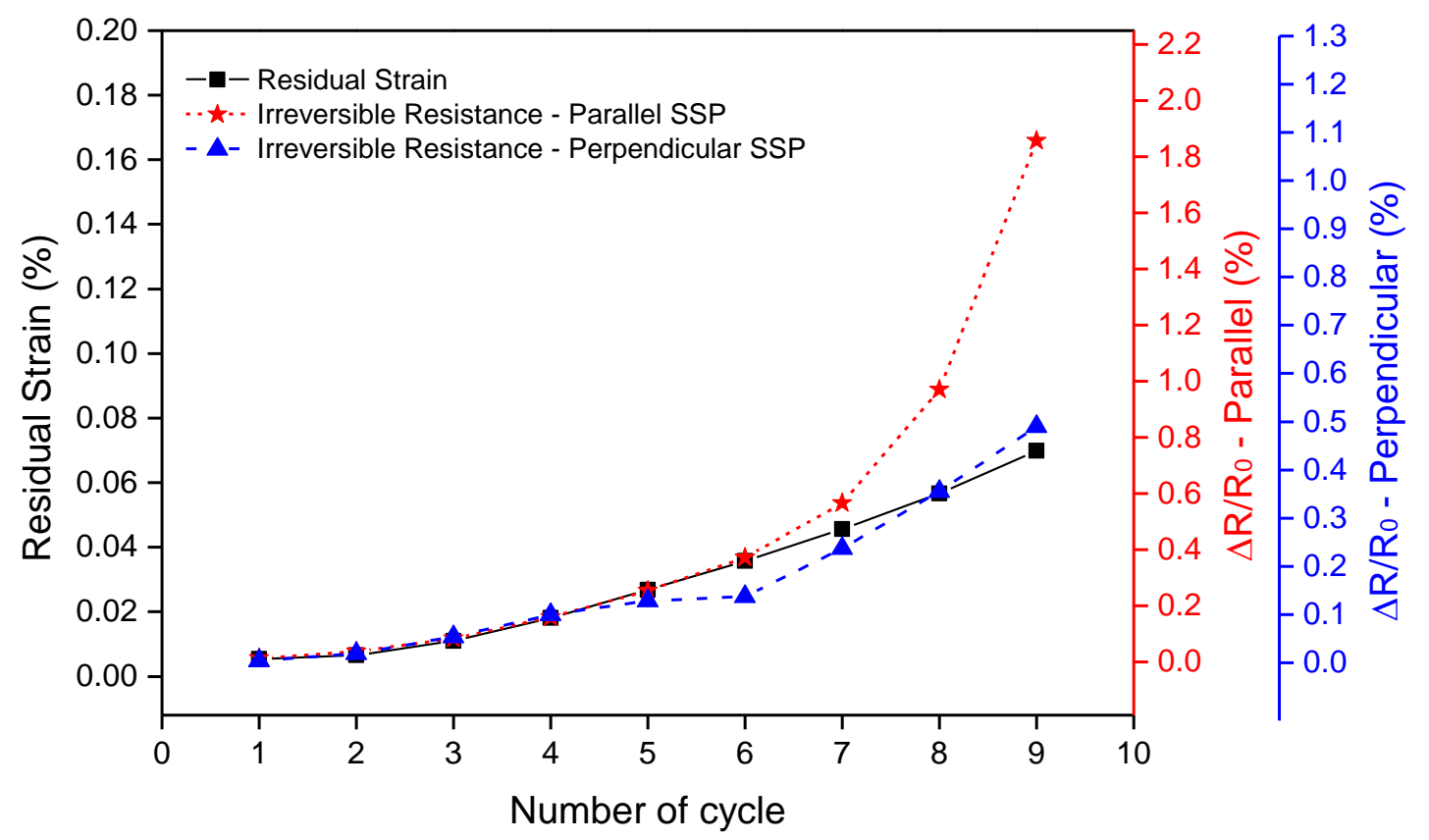

Figure 4.6 Residual axial strain and corresponding irreversible resistance change of parallel and perpendicular SSPs in the progressive damage accumulation tests. A quasilinear increase of the residual strain can be observed, generally matching with the irreversible resistance change progression. Transverse crack induced by high level strain damaged the CNTs network in parallel SSPs more significantly, leading to its curve deviation. (The cycles are identical to those in the progressive damage accumulation tests in Figure 4.4) 
The irreversible resistance change of parallel sensors correlates with the residual strain progression during the first 6 cycles, and then deviates substantially afterwards. While for the perpendicular SSPs, the irreversible resistance change curve for the most part was able to follow the residual strain variations through the loading - unloading cycles. With the increase of the strain applied to the specimens, the number of transverse cracks possibly increases correspondingly. The transverse matrix micro-cracking is perpendicular to CNT orientations in parallel SSPs [50]. Hence, it is believed that the irreversible resistance changed more for parallel SSPs because when multiple nano- and micro- cracks form in transverse direction, some may fracture CNTs and cut off the conductive network locally. Then, at a relative high level of strain, the electrical resistance would increase in a rapid speed and deviate from the former slope. For the case of perpendicular SSPs, the CNTs are effected less by transverse matrix cracks and thus obtain a better correlation of residual strain to irreversible resistance change. In general, very similar behavior for parallel and perpendicular sensors can be seen, meaning that the residual strains could be correlated to irreversible resistance changes in certain strain range $(<1.2 \% \varepsilon$ according to Figure 4.6$)$ regardless of the CNTs orientations of SSPs.

\subsubsection{Dynamic Uniaxial Fatigue Test}

As demonstrated in the former cyclic tensile test, the CNT SSPs showed quite consistent piezoresistive performance during a few cycles of mechanical loading - unloading. Considering the static stability confirmed above, it was expected that adequate dynamic stability and long-time consistency could also be achieved. However, this was not the case. 
Dynamic uniaxial tensile tests of 1000 cycles were performed under $0.4 \%$ and $0.8 \%$ strain to show the resistance change during fatigue loading cycles for the analysis of dynamic stability and consistency. The highly repeatable resistance change patterns (Figure 4.7) under strain rate of $0.4 \%$ for both types of SSPs demonstrated the feasibility of cyclically stable sensing performance within the elastic deformation strain range.

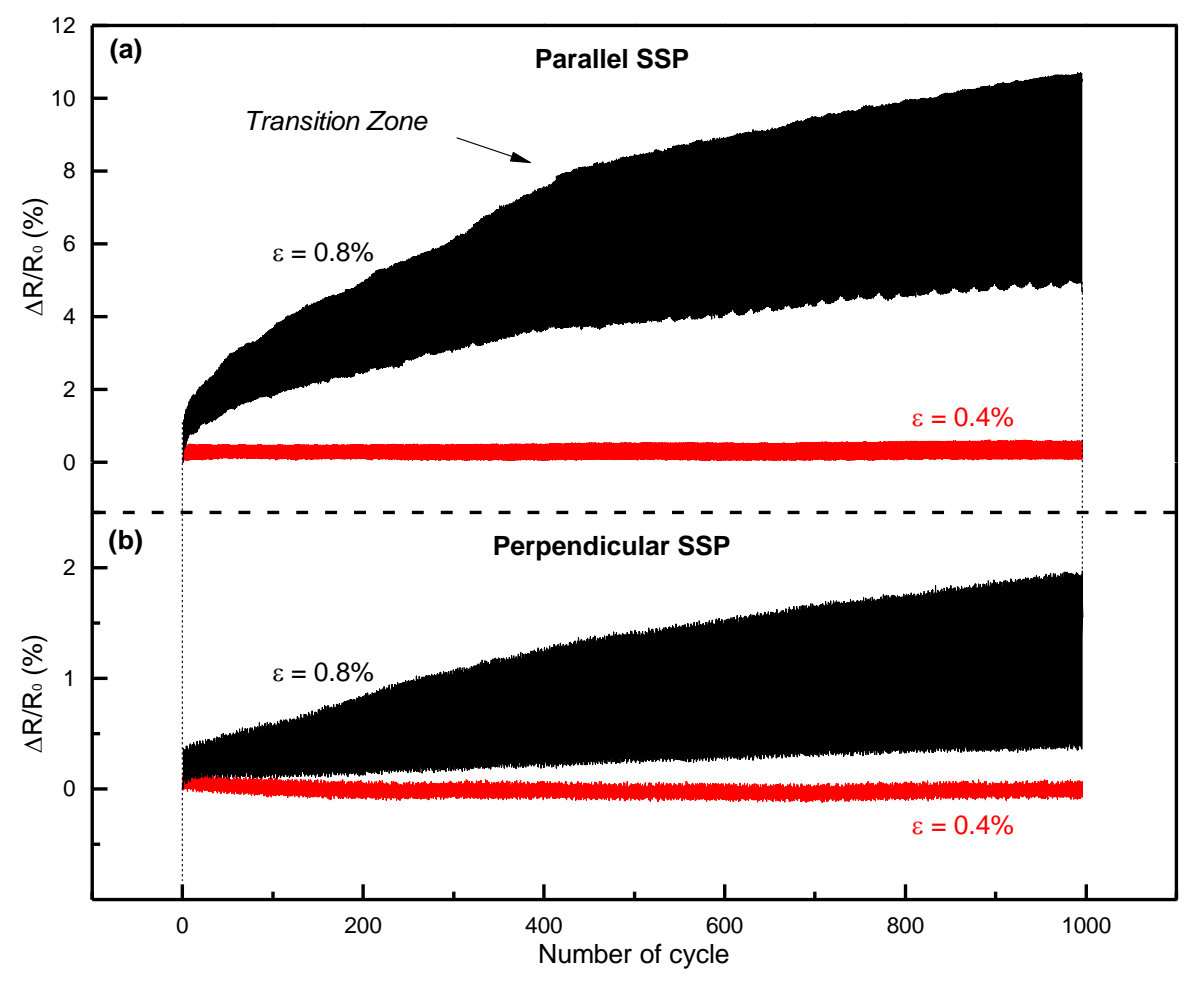

Figure 4.7 Relative resistance change $\left(\Delta R / R_{0}\right)$ response of (a) parallel and (b) perpendicular SSPs stretched to $0.4 \%$ (red) and $0.8 \%$ (black) strain (E) during 1000 cycle long term fatigue test. The $\Delta R / R_{0}$ behavior is stable and consistent for both types of SSPs at $0.4 \% \varepsilon$. The value of $\Delta R / R_{0}$ varied in an expanding range over time at $0.8 \% \varepsilon$, and more obvious $\Delta R / R_{0}$ peak value increase can be observed for parallel SSPs. The $\Delta R / R_{0}$ at zero load increased over time as well. A transition zone occurred after certain numbers of loading - unloading, where all the trends above were attenuated, showing a gradually stabilized piezoresistive behavior. 
When $0.8 \%$ strain was applied, the resistance change at the peak loadings increased and the difference between the resistance change at maximum and minimum load also expanded during the fatigue test. This is possibly caused by the viscoelastic deformation of the composite substrate which certainly induced some permanent damage to the structure of nanotube electrical conductive networks in the SSPs adhered on the surface. As the parallel sensors have more abundant CNTs orientated perpendicular to the transverse cracking, continuous loading and unloading would interfere and fracture the CNTs more easily. In this case, electrical pathways would be more severely damaged with the irreversible deformation of composite substrates. They are both more sensitive and more vulnerable to the strains, suffering a more significant resistance change expansion and baseline shift, shown in Figure 4.7(a). Compared to the parallel sensors, perpendicular SSPs experienced less variations of resistance change expansion and baseline shift. Because the orientations of CNTs were mostly parallel to transverse cracks, the nanotube network would not be damaged as much.

On the other hand, a sharp increase of $\Delta R / R_{0}$ was initially observed within the first several hundred of cycles and afterwards the $\Delta \mathrm{R} / \mathrm{R}_{0}$ increases at lower amplitude. This is defined as a transition zone where a trend of stabilization of the resistance change can be observed as the sensors were stretched more, especially for the case of parallel specimens. It was hypothesized that the CNTs sheet structure would achieve a structural balanced and energy preferential status after sufficient cycles of loading - unloading. Because the cracks would grow in size and amount, then saturation would be gradually achieved in a certain strain range. Importantly, during the 1000 cycle fatigue tensile tests, no extreme increase of 
resistance change had been observed, indicating that the SSPs were still capable to function properly in this strain range. Better repeatability and consistency are expected after a prestabilization process, such as dynamic pre-straining.

For the dynamic fatigue testing, $0.2 \%$ strain was initially tried then $0.4 \%$ and $0.8 \%$ as following. We tried small level strain realizing the fact that our specimens were able to reversibly repeat the linear piezoresistive behavior very well. Then according to the electrical resistance behavior observed in monotonic tensile test, we estimate that the specimens could further withstand $0.8 \%$ strain preserving stable dynamic performances. So we directly tried $0.8 \%$ strain to roughly evaluate the possible upper limit of functional range for our SSPs. The specimens would possibly have more stable performance in the dynamic fatigue test at strain levels between $0.4 \%$ and $0.8 \%$. That can be a crucial part in our future works to more clearly identify the capability of the developed sensors in the aspect of long time SHM applications.

\subsection{Effect of Pre-straining on Sensing Performance}

Overall, the piezoresistive behavior of our CNT SSPs was fairly good for strain sensing utilization at low strain values, yet better performance can be achieved by further engineering the structure. The piezoresistive response of SSP to applied strain in this work can only be described as quasi-linear, which is the same case in many other research studies $[14,21,38,62]$. Thus, more work is needed to make truly applicable and useful strain sensors over a large strain range. In order to achieve better linearity for our samples, we conducted a pre-straining procedure to some parallel SSPs samples. The test coupons with attached CNT SSP parallel specimens were first stretched with a load controlled mode to $56 \mathrm{kN}(\sim 1.2 \% \varepsilon)$ 
and then loaded with a decrement of $7 \mathrm{kN}$ per cycle. After this pre-straining, some of the specimens underwent the quasi-static monotonic tensile test till fracture; some were then tested in 1000 cycle fatigue test with a peak strain of $0.8 \%$ each cycle. The electrical resistance data was in-situ measured during the whole process.

As expected, the piezoresistive response of pre-treated specimens showed much better linearity in the elastic deformation range $(0 \sim 0.8 \% \varepsilon)$, shown in Figure 4.8 .

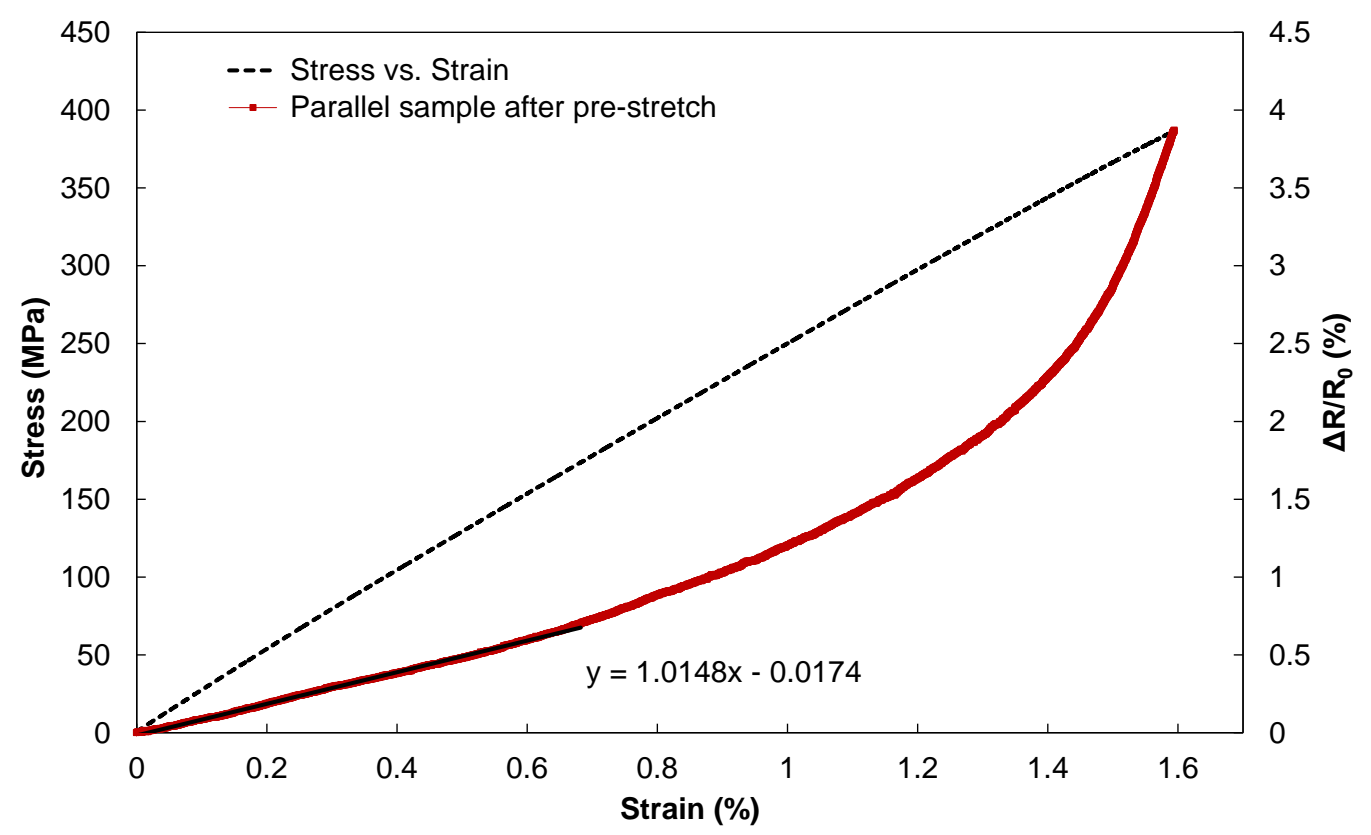

Figure 4.8 Monotonic tensile test after pre-straining, showing better linearity but lower sensitivity (GF of 1$)$ of normalized resistance change $\left(\Delta R / R_{0}\right)$ to strain in the range of $0 \sim 0.7 \%$. Smooth transition from linear to non-linear regime showed better sensing stability of pre-strained specimens. 
As the specimens were pre-strained, the whole structure of the SSP experienced a process of pre-cracking. Since the cracks in the matrix had already permanently changed the CNT network, the SSP should behave in a more stable manner.

A more linear piezoresistive trend was observed in a broadened strain range. From Figure 4.9, the GF for the pre-strained SSP maintained around certain value in the strain range of $0 \sim 1 \%$ and thus showed more stable behavior at different strain levels.

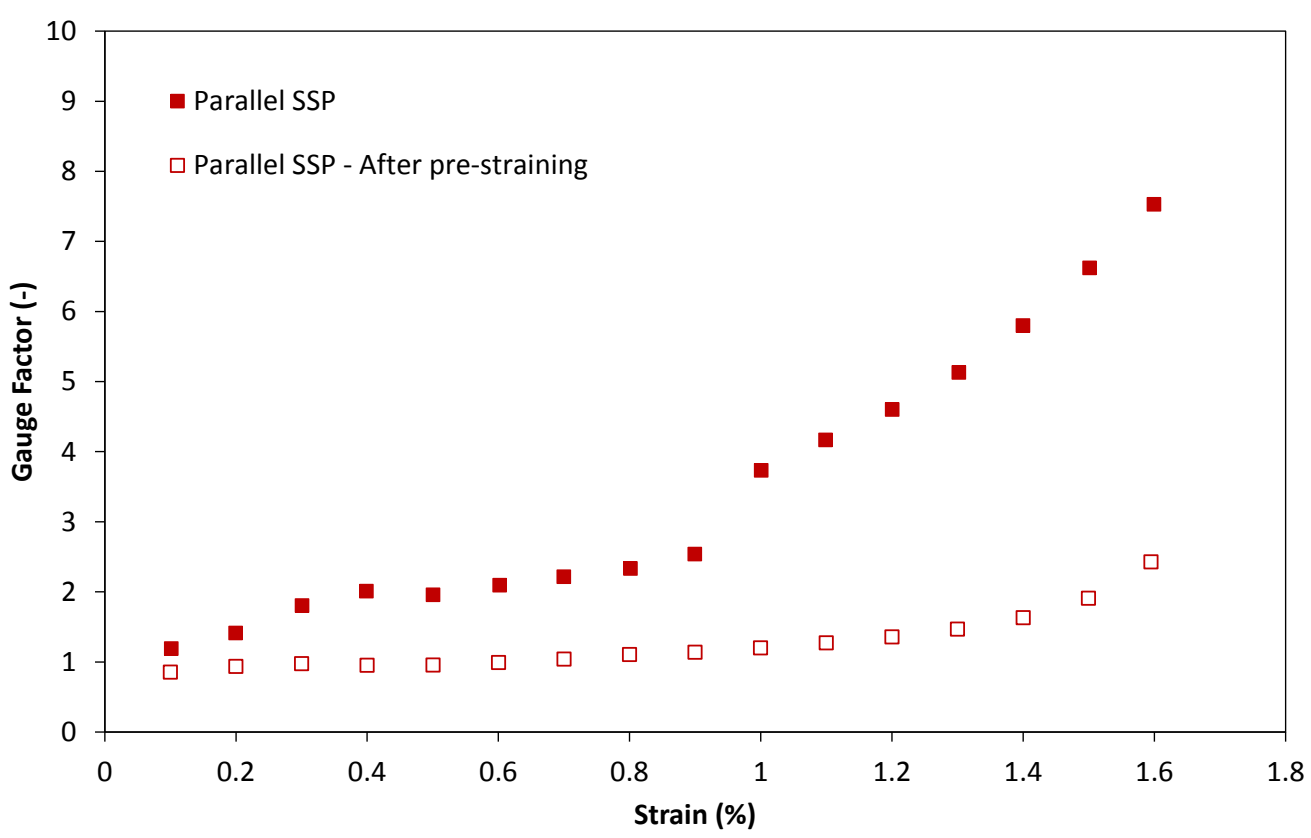

Figure 4.9 Comparison of the GF behavior of parallel SSPs with and without prestraining over a increasing strain level. 
The roughness on the $\Delta \mathrm{R} / \mathrm{R}_{0}$ vs. $\varepsilon$ curve due to the forming and propagating of cracks was also eliminated. Since the cracks were intentionally produced in the pre-straining, there would be neither any new cracks formation nor little additional crack propagation when strained less than $1.2 \%$. In this case, a sudden and drastic increase of $\Delta R / R_{0}$ induced by an abrupt formation of new cracks was able to be avoided. As seen in the Figure 4.8, the "jump" increase of normalized electrical resistance change on the piezoresistive curve (Figure 4.2) disappeared, replaced by a smooth transition from elastic deformation regime to viscoelastic deformation regime of composite coupon substrates. Therefore, the piezoresistive behavior of pre-stretched specimens corresponded to strain variations without extra calibrations in the working range of $0 \sim 0.8 \% \varepsilon$, giving them great potential to function as high-accuracy strain sensors for advanced composite materials. To evaluate the effect of pre-straining on longtime dynamic piezoresistive behavior of the specimens, the data of CNT SSPs without prestraining were plotted altogether in Figure 4.10 as a comparison. 


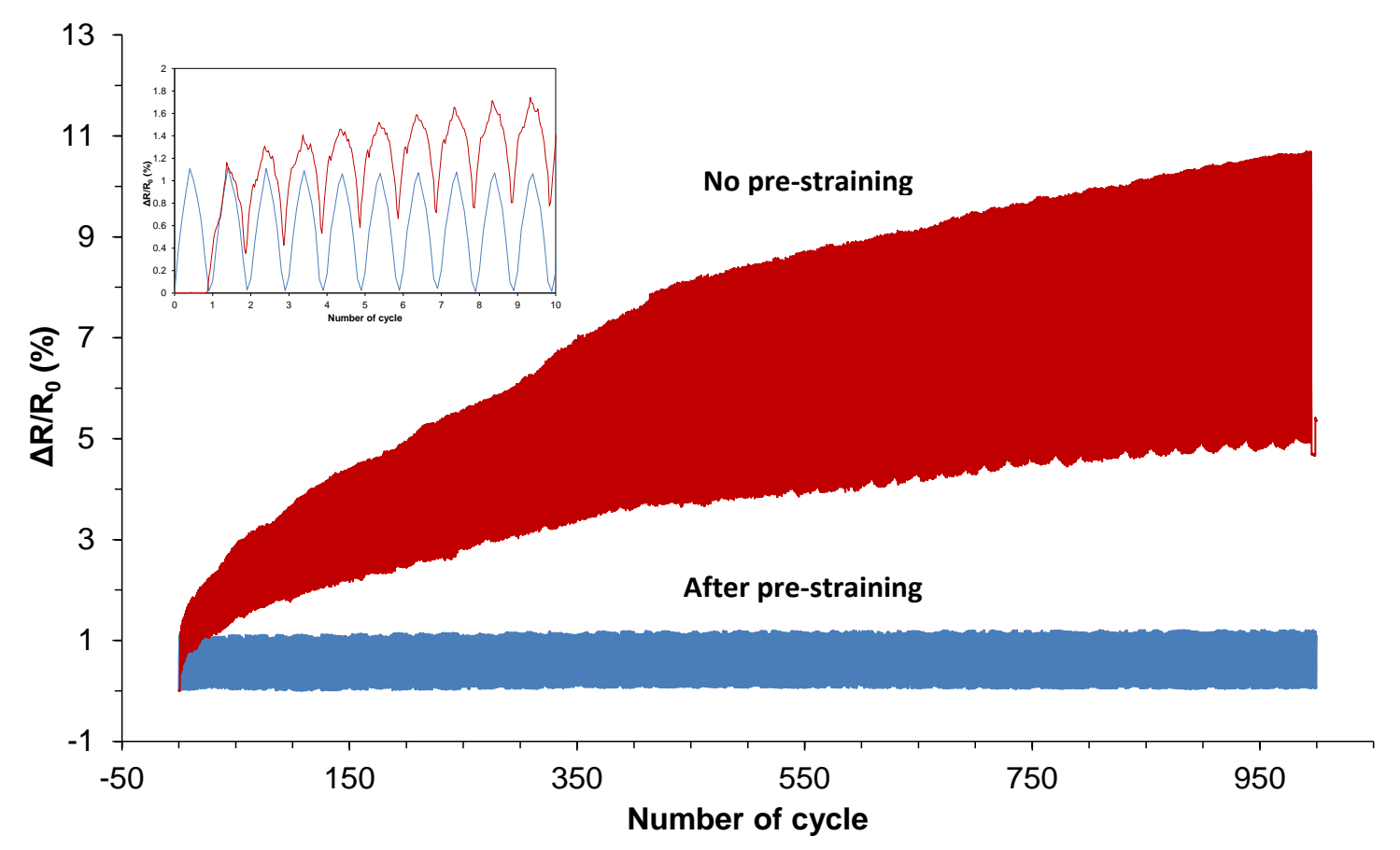

Figure 4.10 Comparison of the piezoresistive response of parallel SSPs without prestraining (red) and after pre-straining to $1.2 \%$ (blue) for 1000 cycle fatigue test. The pre-strained specimens showed better stability and reversibility over long time testing, exhibiting more reliable sensing performance. Inset: close-up of the first 10 cycles of $\Delta R / R_{0}$ response to strain.

Apparently, there is no obvious drift of $\Delta R / R_{0}$ variation all along the continuous cycling of pre-strained CNT SSPs. The variations of $\Delta R / R_{0}$ for pre-strained SSPs were able to be kept in the same range over long-term cyclic loading. Pretty good consistency and durability were exhibited and the linear piezoresistive response was maintained with almost no noise (Figure 4.10 , inset). Therefore, the pre-straining process was proved to be an effective method to enhance the piezoresistive performance for our CNT SSPs. 


\section{Conclusion and Future Work}

\subsection{Research Conclusions}

The aligned CNTs SSPs were fabricated in a fairly simple but effective way, incorporating the strain sensors and the glass fiber composite substrates into a whole. The development of this type of simple and low-cost fabrication technique would favor the integral composite sensing and commercial productions. The electrical signal during tests over time and temperature variations, without mechanical load, showed good intrinsic piezoresistive stability of the aligned nanotube sheet structure and wide sensing compatible temperature range for SSPs. The piezoresistive mechanism of both types of SSPs were attributed to a combination of the intrinsic piezoresistive effect of individual CNTs and variations of CNT contact resistance and tunneling resistance, which are controlled by the strain transfer to CNTs and the initiation, propagation and accumulation of extremely small cracks in the matrix system. Although both parallel and perpendicular CNT SSPs followed a similar electrical resistance response to the applied strain, there was a significant effect of nanotube orientation on sensor sensitivity, showing distinct GF in the range of 1 8 and $0.5 \sim 1.3$ for parallel and perpendicular SSPs respectively. Thus, the parallel SSPs with high GF are more suited for sensing applications. This anisotropic sensing performance may also be useful to determine the direction of the applied load if multiple sensors are used. The piezoresistive response of the CNT SSPs during 1000 cycle dynamic fatigue test displayed significant drift over time, revealing continuous damage to the conductive nanotube network. To optimize the piezoresistive performance of the CNT SSPs, a pre-straining process was applied rendering a 
more consistent linear behavior over an even larger strain range. With the addition of additional engineering optimization of the sensor configuration and structure, this new technique may be useful for commercial multifunctional sensors for real-time composite strain and damage sensing.

\subsection{Recommendation for Future Work}

- The relationship between pre-straining and sensor performance should be further investigated. The patch should be cured by itself and then strained before adhering it to a composite structure. In this way the patch could be strained even further than $1.2 \%$ without damaging the underlying composite test coupon. After pre-straining the patch should then be adhered to the composite test coupon.

- Compression or bending tests need to be done to understand the sensing behavior under compressive loading conditions. An addition of one more strain gauge would enable the real-time sensing of both tensile and compressive behavior altogether. It will be interesting to observe the piezoresistive curve of a sample in bending with both tension and compression on the opposite faces.

- The CNT sheets should be embedded into composite structures. Prepregs could be used, embedding the CNTs sheet in-between the prepreg layers. This type of sensor would have the ability to increase the mechanical properties of the composite, such as fracture toughness will perform integral sensing. In addition, with the CNT sheets running the length of the composites, damage detection, especially right before composite failure should become very sensitive. Creating a multi-layer structure and 
individually sensing each layer could provide information about which layer has received damage.

- Multiple CNT sheet layers could be used instead of single layer. In other works thicker and more conductive samples often gave higher sensitivity. This could hold true for our material as well.

- More than hundreds and thousands of SSPs will be needed to achieve integral systematic damage sensing for large composite structures, e.g. bridges. Therefore, it is much more difficult and complicated to establish a properly functional system for large scale global SHM. Due to the geometry nature, our CNT sheet strain sensors can be easily embedded into two-dimensional composite structures but not for composites with three-dimensional textile structures. These limitations would drive the future study on the capability and appropriate applications of this type of novel CNT based strain sensors. 


\section{REFERENCES}

[1] Thostenson ET, Ren Z, Chou T. Advances in the science and technology of carbon nanotubes and their composites: a review. Composites Sci Technol 2001;61:1899-912.

[2] Zhao H, Yuan F. Carbon nanotube yarn sensors for structural health monitoring of composites. 2011:79830P,79830P-7.

[3] Abot JL, Song Y, Vatsavaya MS, Medikonda S, Kier Z, Jayasinghe C et al. Delamination detection with carbon nanotube thread in self-sensing composite materials. Composites Sci Technol 2010;70:1113-9.

[4] Njuguna MK. Characterisation of multi wall carbon nanotube-polymer composites for strain sensing applications. 2012.

[5] Wen J, Xia Z, Choy F. Damage detection of carbon fiber reinforced polymer composites via electrical resistance measurement. Composites Part B: Engineering 2011;42:77-86.

[6] Jin H. Carbon nanotube strain sensors with wide dynamic range fabricated on flexible substrates by novel processing techniques 2009 .

[7] Takeda S, Okabe Y, Takeda N. Delamination detection in CFRP laminates with embedded small-diameter fiber Bragg grating sensors. Composites Part A: Applied Science and Manufacturing 2002;33:971-80.

[8] Udd E. Overview of fiber optic sensors. : New York: Marcel Dekker, 2002.

[9] Loh KJ. Development of multifunctional carbon nanotube nanocomposite sensors for structural health monitoring. : ProQuest, 2008.

[10] FIEDLER B, GOJNY FH, WICHMANN MH, BAUHOFER W, SCHULTE K. Can carbon nanotubes be used to sense damage in composites? 2004;29:81-94.

[11] Thostenson ET, Chou T. Carbon nanotube networks: sensing of distributed strain and damage for life prediction and self healing. Adv Mater 2006;18:2837-41.

[12] Thostenson ET, Chou T. Real-time in situ sensing of damage evolution in advanced fiber composites using carbon nanotube networks. Nanotechnology 2008;19:215713.

[13] Zhao H, Zhang Y, Bradford PD, Zhou Q, Jia Q, Yuan F et al. Carbon nanotube yarn strain sensors. Nanotechnology 2010;21:305502. 
[14] Hehr A, Schulz M, Shanov V, Song Y. Embedded carbon nanotube thread piezoresistive strain sensor performance. Sens Rev 2014;34:209-19.

[15] Dharap P, Li Z, Nagarajaiah S, Barrera E. Nanotube film based on single-wall carbon nanotubes for strain sensing. Nanotechnology 2004;15:379.

[16] Li X, Levy C, Elaadil L. Multiwalled carbon nanotube film for strain sensing. Nanotechnology 2008;19:045501.

[17] Li Z, Dharap P, Nagarajaiah S, Barrera EV, Kim J. Carbon nanotube film sensors. Adv Mater 2004;16:640-3.

[18] Vemuru S, Wahi R, Nagarajaiah S, Ajayan P. Strain sensing using a multiwalled carbon nanotube film. The Journal of Strain Analysis for Engineering Design 2009;44:555-62.

[19] Alexopoulos N, Bartholome C, Poulin P, Marioli-Riga Z. Damage detection of glass fiber reinforced composites using embedded PVA-carbon nanotube (CNT) fibers. Composites Sci Technol 2010;70:1733-41.

[20] Alexopoulos N, Bartholome C, Poulin P, Marioli-Riga Z. Structural health monitoring of glass fiber reinforced composites using embedded carbon nanotube (CNT) fibers. Composites Sci Technol 2010;70:260-71.

[21] Alexopoulos N, Jaillet C, Zakri C, Poulin P, Kourkoulis S. Improved strain sensing performance of glass fiber polymer composites with embedded pre-stretched polyvinyl alcohol-carbon nanotube fibers. Carbon 2013;59:65-75.

[22] Kang TJ, Choi A, Kim D, Jin K, Seo DK, Jeong DH et al. Electromechanical properties of CNT-coated cotton yarn for electronic textile applications. Smart Mater Struct 2011;20:015004.

[23] Sebastian J, Schehl N, Bouchard M, Boehle M, Li L, Lagounov A et al. Health monitoring of structural composites with embedded carbon nanotube coated glass fiber sensors. Carbon 2014;66:191-200.

[24] Zhang J, Liu J, Zhuang R, Mäder E, Heinrich G, Gao S. Single MWNT-Glass Fiber as Strain Sensor and Switch. Adv Mater 2011;23:3392-7.

[25] Bautista-Quijano J, Aviles F, Aguilar J, Tapia A. Strain sensing capabilities of a piezoresistive MWCNT-polysulfone film. Sensors and Actuators A: Physical 2010;159:135-40. 
[26] Bu L, Steitz J, Kanoun O. Influence of processing parameters on properties of strain sensors based on carbon nanotube films. 2010:1-6.

[27] Fan Q, Qin Z, Gao S, Wu Y, Pionteck J, Mäder E et al. The use of a carbon nanotube layer on a polyurethane multifilament substrate for monitoring strains as large as $400 \%$. Carbon 2012;50:4085-92.

[28] Feng Q, Yang J, Fu S, Mai Y. Synthesis of carbon nanotube/epoxy composite films with a high nanotube loading by a mixed-curing-agent assisted layer-by-layer method and their electrical conductivity. Carbon 2010;48:2057-62.

[29] Ferrreira A, Rocha J, Ansón-Casaos A, Martínez M, Vaz F, Lanceros-Mendez S. Electromechanical performance of poly (vinylidene fluoride)/carbon nanotube composites for strain sensor applications. Sensors and Actuators A: Physical 2012;178:10-6.

[30] Hu B, Hu N, Cai Y, Yuan W, Yan C, Cai Y et al. Performance characterization of VGCF/epoxy nanocomposite sensors under static load cycles and in static structural health monitoring. Smart Mater Struct 2013;22:045008.

[31] Hu N, Karube Y, Arai M, Watanabe T, Yan C, Li Y et al. Investigation on sensitivity of a polymer/carbon nanotube composite strain sensor. Carbon 2010;48:680-7.

[32] Kang I, Schulz MJ, Kim JH, Shanov V, Shi D. A carbon nanotube strain sensor for structural health monitoring. Smart Mater Struct 2006;15:737.

[33] Lee C, Jug L, Meng E. High strain biocompatible polydimethylsiloxane-based conductive graphene and multiwalled carbon nanotube nanocomposite strain sensors. Appl Phys Lett 2013;102:183511.

[34] Loh KJ, Lynch JP, Kotov NA. Conformable single-walled carbon nanotube thin film strain sensors for structural monitoring. 2005:1-8.

[35] Loh KJ, Kim J, Lynch JP, Kam NWS, Kotov NA. Multifunctional layer-by-layer carbon nanotube-polyelectrolyte thin films for strain and corrosion sensing. Smart Mater Struct 2007; 16:429.

[36] Loyola BR, Zhao Y, Loh KJ, La Saponara V. The electrical response of carbon nanotube-based thin film sensors subjected to mechanical and environmental effects. Smart Mater Struct 2013;22:025010. 
[37] Luo S, Liu T. Structure-property-processing relationships of single-wall carbon nanotube thin film piezoresistive sensors. Carbon 2013;59:315-24.

[38] Njuguna M, Yan C, Hu N, Bell J, Yarlagadda P. Sandwiched carbon nanotube film as strain sensor. Composites Part B: Engineering 2012;43:2711-7.

[39] Park M, Kim H, Youngblood JP. Strain-dependent electrical resistance of multi-walled carbon nanotube/polymer composite films. Nanotechnology 2008;19:055705.

[40] Pham GT, Park Y, Liang Z, Zhang C, Wang B. Processing and modeling of conductive thermoplastic/carbon nanotube films for strain sensing. Composites Part B: Engineering 2008;39:209-16.

[41] Rizvi R, Naguib HE. Carbon nanotube network evolution during deformation of PVDFMWNT nanocomposites. 2013:86871V,86871V-8.

[42] Song X, Liu S, Gan Z, Lv Q, Cao H, Yan H. Controllable fabrication of carbon nanotube-polymer hybrid thin film for strain sensing. Microelectronic Engineering 2009;86:2330-3.

[43] Wang Y, Wang AX, Wang Y, Chyu MK, Wang Q. Fabrication and characterization of carbon nanotube-polyimide composite based high temperature flexible thin film piezoresistive strain sensor. Sensors and Actuators A: Physical 2013;199:265-71.

[44] Zhang R, Deng H, Valenca R, Jin J, Fu Q, Bilotti E et al. Strain sensing behaviour of elastomeric composite films containing carbon nanotubes under cyclic loading. Composites Sci Technol 2013;74:1-5.

[45] Zhang W, Suhr J, Koratkar N. Carbon nanotube/polycarbonate composites as multifunctional strain sensors. Journal of Nanoscience and Nanotechnology 2006;6:9604.

[46] Liu Y, Chakrabartty S, Gkinosatis DS, Mohanty AK, Lajnef N. Multi-walled carbon nanotubes/poly (l-lactide) nanocomposite strain sensor for biomechanical implants. 2007:119-22.

[47] De la Vega A, Kinloch I, Young R, Bauhofer W, Schulte K. Simultaneous global and local strain sensing in SWCNT-epoxy composites by Raman and impedance spectroscopy. Composites Sci Technol 2011;71:160-6. 
[48] de la Vega A, Sumfleth J, Wittich H, Schulte K. Time and temperature dependent piezoresistance of carbon nanofiller/polymer composites under dynamic load. J Mater Sci 2012;47:2648-57.

[49] Ferreira A, Cardoso P, Klosterman D, Covas J, Van Hattum F, Vaz F et al. Effect of filler dispersion on the electromechanical response of epoxy/vapor-grown carbon nanofiber composites. Smart Mater Struct 2012;21:075008.

[50] Gao L, Thostenson ET, Zhang Z, Chou T. Sensing of Damage Mechanisms in FiberReinforced Composites under Cyclic Loading using Carbon Nanotubes. Advanced Functional Materials 2009;19:123-30.

[51] Knite M, Tupureina V, Fuith A, Zavickis J, Teteris V. Polyisoprene-multi-wall carbon nanotube composites for sensing strain. Materials Science and Engineering: C 2007;27:1125-8.

[52] Ku-Herrera J, Avilés F. Cyclic tension and compression piezoresistivity of carbon nanotube/vinyl ester composites in the elastic and plastic regimes. Carbon 2012;50:2592-8.

[53] Ku-Herrera J, Avilés F, Seidel G. Self-sensing of elastic strain, matrix yielding and plasticity in multiwall carbon nanotube/vinyl ester composites. Smart Mater Struct 2013;22:085003.

[54] Li C, Chou T. Modeling of damage sensing in fiber composites using carbon nanotube networks. Composites Sci Technol 2008;68:3373-9.

[55] Nofar M, Hoa S, Pugh M. Failure detection and monitoring in polymer matrix composites subjected to static and dynamic loads using carbon nanotube networks. Composites Sci Technol 2009;69:1599-606.

[56] Saafi M. Wireless and embedded carbon nanotube networks for damage detection in concrete structures. Nanotechnology 2009;20:395502.

[57] Böger L, Wichmann MH, Meyer LO, Schulte K. Load and health monitoring in glass fibre reinforced composites with an electrically conductive nanocomposite epoxy matrix. Composites Sci Technol 2008;68:1886-94.

[58] Wichmann MH, Buschhorn ST, Böger L, Adelung R, Schulte K. Direction sensitive bending sensors based on multi-wall carbon nanotube/epoxy nanocomposites. Nanotechnology 2008;19:475503. 
[59] Wichmann MH, Buschhorn ST, Gehrmann J, Schulte K. Piezoresistive response of epoxy composites with carbon nanoparticles under tensile load. Physical Review B 2009;80:245437.

[60] Zetina-Hernández O, Duarte-Aranda S, May-Pat A, Canché-Escamilla G, UribeCalderon J, Gonzalez-Chi P et al. Coupled electro-mechanical properties of multiwall carbon nanotube/polypropylene composites for strain sensing applications. J Mater Sci 2013;48:7587-93.

[61] Zha J, Shehzad K, Li W, Dang Z. The effect of aspect ratio on the piezoresistive behavior of the multiwalled carbon nanotubes/thermoplastic elastomer nanocomposites. J Appl Phys 2013;113:014102.

[62] Oliva-Avilés A, Avilés F, Sosa V. Electrical and piezoresistive properties of multiwalled carbon nanotube/polymer composite films aligned by an electric field. Carbon 2011;49:2989-97.

[63] Theodosiou T, Saravanos D. Numerical investigation of mechanisms affecting the piezoresistive properties of CNT-doped polymers using multi-scale models. Composites Sci Technol 2010;70:1312-20.

[64] Zhou X. Carbon nanotube transistors, sensors, and beyond. , 2008.

[65] Gayathri V, Geetha R. Carbon nanotube as NEMS sensor-effect of chirality and stonewales defect intend. 2006;34:824.

[66] Meyyappan M. Carbon nanotubes: science and applications. : CRC press, 2004.

[67] Baughman RH, Zakhidov AA, de Heer WA. Carbon nanotubes--the route toward applications. Science 2002;297:787-92.

[68] Bradford PD. Mechanical Characterization and Morphology Study of a Novel Class of Carbon Nanotube Textiles and Composites. 2007.

[69] Inoue Y, Kakihata K, Hirono Y, Horie T, Ishida A, Mimura H. One-step grown aligned bulk carbon nanotubes by chloride mediated chemical vapor deposition. Appl Phys Lett 2008;92:213113.

[70] Hata K, Futaba DN, Mizuno K, Namai T, Yumura M, Iijima S. Water-assisted highly efficient synthesis of impurity-free single-walled carbon nanotubes. Science 2004;306:1362-4. 
[71] Zhang L, Tan Y, Resasco DE. Controlling the growth of vertically oriented singlewalled carbon nanotubes by varying the density of CoMo catalyst particles. Chemical physics letters 2006;422:198-203.

[72] Zhang W, Wen Y, Tjiu W, Xu G, Gan L. Growth of vertically aligned carbon-nanotube array on large area of quartz plates by chemical vapor deposition. Applied Physics A 2002;74:419-22.

[73] Yun Y, Shanov V, Tu Y, Subramaniam S, Schulz MJ. Growth mechanism of long aligned multiwall carbon nanotube arrays by water-assisted chemical vapor deposition. The Journal of Physical Chemistry B 2006;110:23920-5.

[74] Yamada T, Namai T, Hata K, Futaba DN, Mizuno K, Fan J et al. Size-selective growth of double-walled carbon nanotube forests from engineered iron catalysts. Nature Nanotechnology 2006;1:131-6.

[75] Zhang H, Cao G, Wang Z, Yang Y, Shi Z, Gu Z. Influence of ethylene and hydrogen flow rates on the wall number, crystallinity, and length of millimeter-long carbon nanotube array. The Journal of Physical Chemistry C 2008;112:12706-9.

[76] Yildiz O, Bradford PD. Aligned carbon nanotube sheet high efficiency particulate air filters. Carbon 2013;64:295-304.

[77] Liu K, Sun Y, Chen L, Feng C, Feng X, Jiang K et al. Controlled growth of superaligned carbon nanotube arrays for spinning continuous unidirectional sheets with tunable physical properties. Nano letters 2008;8:700-5.

[78] Atkinson KR, Hawkins SC, Huynh C, Skourtis C, Dai J, Zhang M et al. Multifunctional carbon nanotube yarns and transparent sheets: fabrication, properties, and applications. Physica B: Condensed Matter 2007;394:339-43.

[79] Zhang X, Jiang K, Feng C, Liu P, Zhang L, Kong J et al. Spinning and Processing Continuous Yarns from 4-Inch Wafer Scale Super-Aligned Carbon Nanotube Arrays. Adv Mater 2006;18:1505-10.

[80] Li Q, Zhang X, DePaula RF, Zheng L, Zhao Y, Stan L et al. Sustained growth of ultralong carbon nanotube arrays for fiber spinning. Adv Mater 2006;18:3160-3.

[81] Pöhls J, Johnson MB, White MA, Malik R, Ruff B, Jayasinghe C et al. Physical properties of carbon nanotube sheets drawn from nanotube arrays. Carbon 2012;50:4175-83. 
[82] Zhang X, Li Q, Holesinger TG, Arendt PN, Huang J, Kirven PD et al. Ultrastrong, Stiff, and Lightweight Carbon-Nanotube Fibers. Adv Mater 2007;19:4198-201.

[83] Aliev AE, Guthy C, Zhang M, Fang S, Zakhidov AA, Fischer JE et al. Thermal transport in MWCNT sheets and yarns. Carbon 2007;45:2880-8.

[84] Obitayo W, Liu T. A review: Carbon nanotube-based piezoresistive strain sensors. Journal of Sensors 2012;2012.

[85] Tombler TW, Zhou C, Alexseyev L, Kong J, Dai H, Liu L et al. Reversible electromechanical characteristics of carbon nanotubes under local-probe manipulation. Nature 2000;405:769-72.

[86] Stampfer C, Jungen A, Linderman R, Obergfell D, Roth S, Hierold C. Nanoelectromechanical displacement sensing based on single-walled carbon nanotubes. Nano letters 2006;6:1449-53.

[87] Luo S. Processing-Structure-Property Relationships Of Carbon Nanotube And Nanoplatelet Enabled Piezoresistive Sensors. 2013.

[88] Liu K, Sun Y, Liu P, Lin X, Fan S, Jiang K. Cross-Stacked Superaligned Carbon Nanotube Films for Transparent and Stretchable Conductors. Advanced Functional Materials 2011;21:2721-8.

[89] Wu J, Zang J, Larade B, Guo H, Gong X, Liu F. Computational design of carbon nanotube electromechanical pressure sensors. PHYSICAL REVIEW-SERIES B2004;69:153406-.

[90] Zhang R, Baxendale M, Peijs T. Universal resistivity-strain dependence of carbon nanotube/polymer composites. Physical Review B 2007;76:195433.

[91] Dang Z, Jiang M, Xie D, Yao S, Zhang L, Bai J. Supersensitive linear piezoresistive property in carbon nanotubes/silicone rubber nanocomposites. J Appl Phys 2008;104:024114.

[92] Gau C, Ko H, Chen H. Piezoresistive characteristics of MWNT nanocomposites and fabrication as a polymer pressure sensor. Nanotechnology 2009;20:185503.

[93] Hwang J, Jang J, Hong K, Kim KN, Han JH, Shin K et al. Poly (3-hexylthiophene) wrapped carbon nanotube/poly (dimethylsiloxane) composites for use in finger-sensing piezoresistive pressure sensors. Carbon 2011;49:106-10. 
[94] Hu C, Liu C, Chen L, Peng Y, Fan S. Resistance-pressure sensitivity and a mechanism study of multiwall carbon nanotube networks/poly (dimethylsiloxane) composites. Appl Phys Lett 2008;93:033108.

[95] Yin G, Hu N, Karube Y, Liu Y, Li Y, Fukunaga H. A carbon nanotube/polymer strain sensor with linear and anti-symmetric piezoresistivity. J Composite Mater 2011;45:1315-23.

[96] Hu N, Karube Y, Yan C, Masuda Z, Fukunaga H. Tunneling effect in a polymer/carbon nanotube nanocomposite strain sensor. Acta Materialia 2008;56:2929-36.

[97] Frogley M, Zhao Q, Wagner H. Polarized resonance Raman spectroscopy of single-wall carbon nanotubes within a polymer under strain. Physical Review B 2002;65:113413.

[98] Maune H, Bockrath M. Elastomeric carbon nanotube circuits for local strain sensing. Appl Phys Lett 2006;89:173131.

[99] Prepreg 7781 E-Glass Product Data Sheet. 2010;2014:1.

[100] Standard A. D3039/D3039M-08, 2008, "Standard Test Method for Tensile Properties of Polymer Matrix Composite Materials" ASTM International, West Conshohocken, PA, 2007, DOI: 10.1520/D3039_D3039M-08.

[101] Koratkar N, Modi A, Lass E, Ajayan P. Temperature effects on resistance of aligned multiwalled carbon nanotube films. Journal of nanoscience and nanotechnology 2004;4:744-8.

[102] Zhang M, Fang S, Zakhidov AA, Lee SB, Aliev AE, Williams CD et al. Strong, transparent, multifunctional, carbon nanotube sheets. Science 2005;309:1215-9.

[103] Wang D, Song P, Liu C, Wu W, Fan S. Highly oriented carbon nanotube papers made of aligned carbon nanotubes. Nanotechnology 2008;19:075609.

[104] Zhang Y, Sheehan CJ, Zhai J, Zou G, Luo H, Xiong J et al. Polymer-Embedded Carbon Nanotube Ribbons for Stretchable Conductors. Adv Mater 2010;22:3027-31.

[105] Gao L, Thostenson ET, Zhang Z, Chou T. Coupled carbon nanotube network and acoustic emission monitoring for sensing of damage development in composites. Carbon 2009;47:1381-8. 
[106] Ogasawara T, Moon S, Inoue Y, Shimamura Y. Mechanical properties of aligned multi-walled carbon nanotube/epoxy composites processed using a hot-melt prepreg method. Composites Sci Technol 2011;71:1826-33.

[107] Fu S, Lauke B, Mäder E, Yue C, Hu X. Tensile properties of short-glass-fiber-and short-carbon-fiber-reinforced polypropylene composites. Composites Part A: Applied Science and Manufacturing 2000;31:1117-25.

[108] Simmons JG. Electric tunnel effect between dissimilar electrodes separated by a thin insulating film. J Appl Phys 2004;34:2581-90.

[109] Zhang X, Pan Y, Zheng Q, Yi X. Time dependence of piezoresistance for the conductor-filled polymer composites. Journal of Polymer Science Part B: Polymer Physics 2000;38:2739-49. 


\section{Appendix}

In the appendix, preliminary work is presented covering the data of some initial trial testing, e.g. monotonic tensile testing, constant strain cyclic testing at different strain levels. The results from the trial testing prompt the modification of mechanical testing and data acquisition methods. The testing coupons without tabs were found fracturing at very low strain level due to the stress concentrations at the grip of the tensile testing machine. Thus, tabs were added to new samples and helped to improve the testing accuracy in the aspect of reducing the sample damage from tensile testing machine. The former method to collect realtime resistance data was limited by the maximum number and minimum frequency of data acquisition. The new software from Agilent technology helped to enhance the data collection capability and thus build up an in situ electrical data acquisition system for the testing hereafter. By doing and analyzing preliminary testing, proper adjustment can then be made to avoid the improper work and gradually shape the necessary core experimentations for this research. 


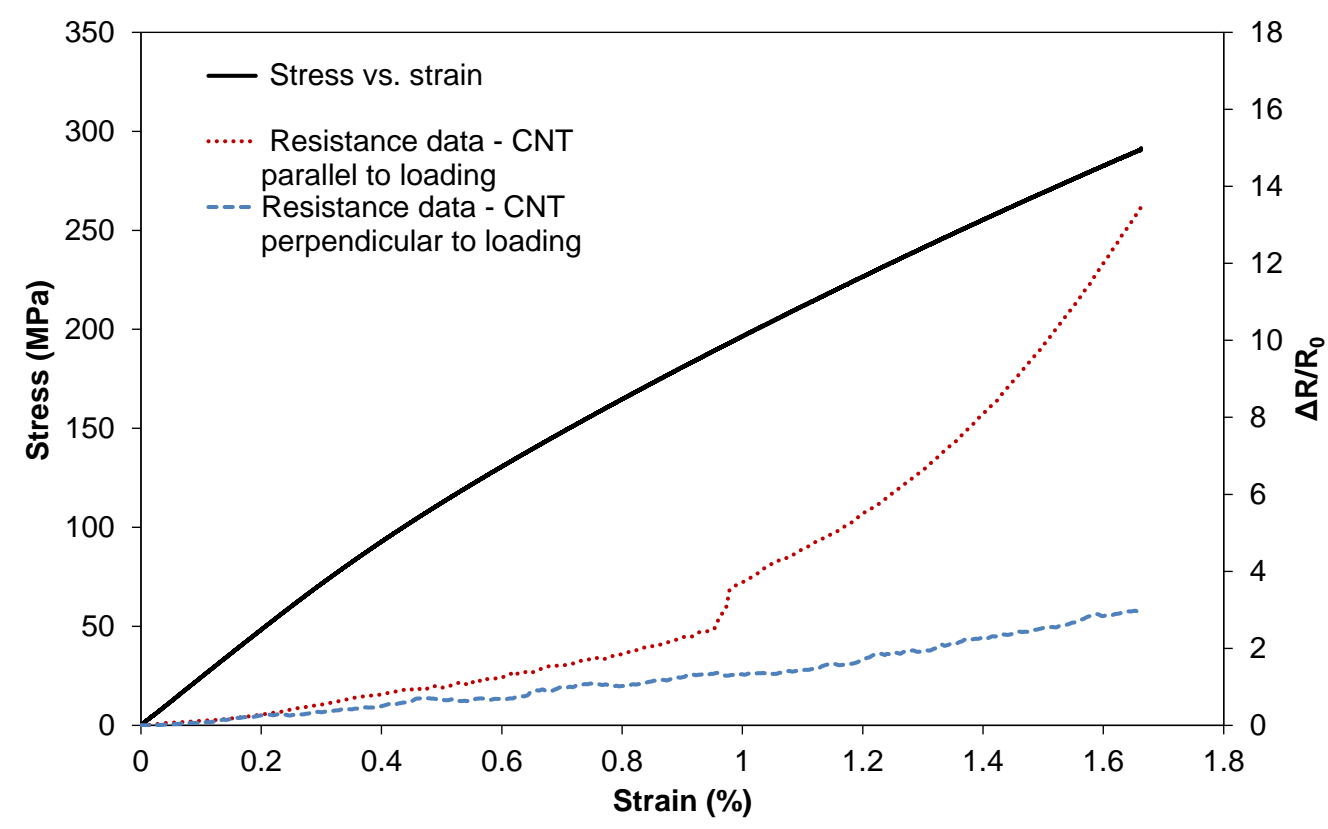

Figure 6.1 The mechanical stress/strain curve of monotonic tensile test and piezoresistive curve of parallel and perpendicular SSP, showing good similarity with Figure 4.2.

Figure 6.1 presented the results of monotonic tensile testing of another two parallel and perpendicular SSPs. The resistance data generally behaved quite similar to the trend analyzed in the results and discussion section. The perpendicular SSP showed slightly higher sensitivity, but not as good linearity of the piezoresistive correlation with many obvious upand-downs on the curve. 


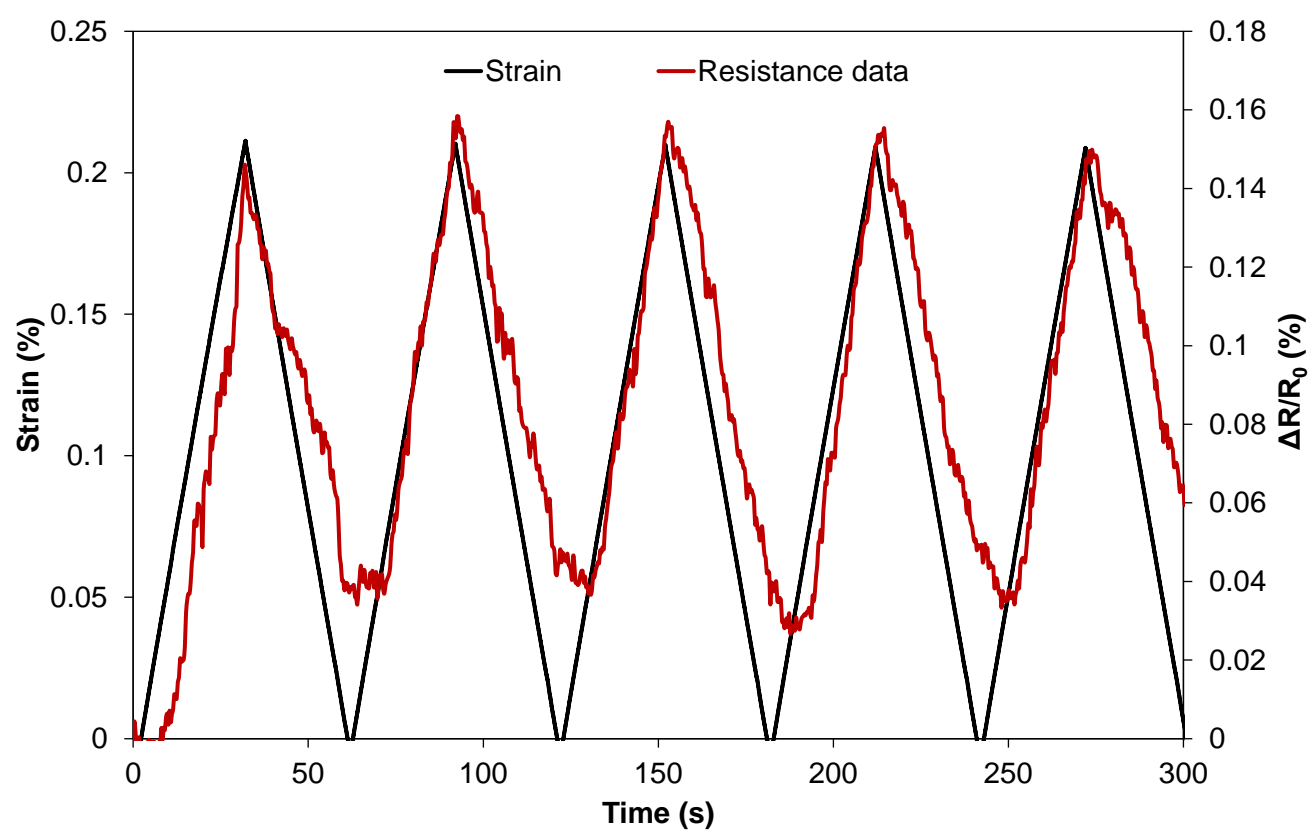

Figure 6.2 Constant strain cyclic tensile test: normalized electrical resistance change of parallel SSP versus time in response to 5 cycles of loading - unloading of $0.2 \%$ strain.

The resistance change curve showed roughness due to noise effect, but generally displaying good quasi-linear trend in loading cycles. The piezoresistive performance was not as good during the unloading cycles. At $0.2 \%$ strain, the electrical resistance data were able to follow the strain data fairly well. Therefore, we conducted constant strain cyclic tensile testing with higher strain values at $0.4 \%$, as shown in Figure 6.3 . 


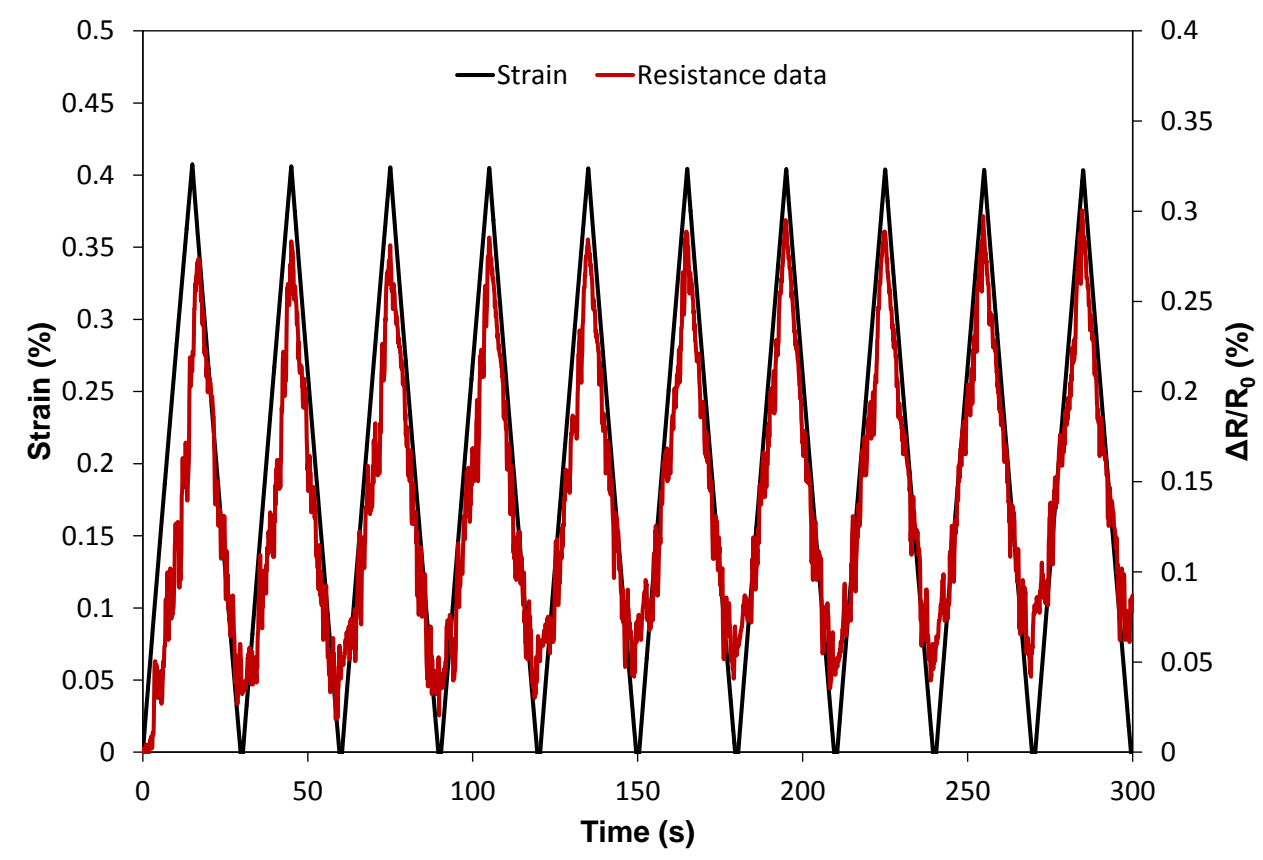

Figure 6.3 Constant strain cyclic tensile test: normalized electrical resistance change of parallel SSP versus time in response to 10 cycles of loading - unloading of $0.4 \%$ strain.

The fairly good quasi-linear piezoresistive trend was preserved within 10 cycles of loading unloading, with slight increase of $\Delta R / R_{0}$ at the last 2 cycles. Since quite consistent quasilinear behavior of the piezoresistive curve was observed all the way to $\sim 0.95 \%$ in the monotonic testing, we directly applied the cyclic loading - unloading with a maximum strain of $0.8 \%$ for each cycle without trying the strain values between $0.4 \%$ and $0.8 \%$. As proved in the results and discussion section above, our SSPs can work repeatedly with a quasi-linear electrical data change in respond to the strain variation accordingly. 


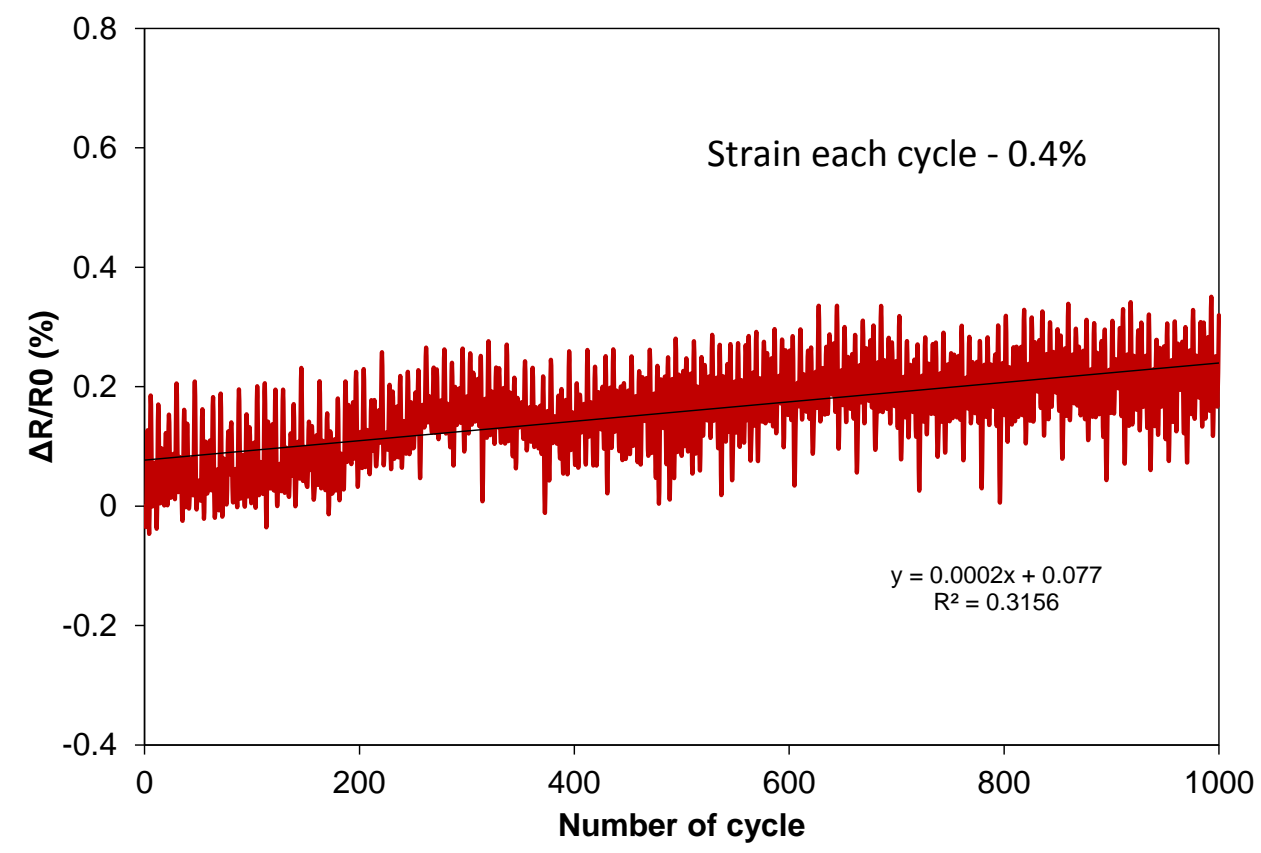

Figure 6.4 Constant strain cyclic tensile test: normalized electrical resistance change of parallel SSP versus time in response to 1000 cycles of loading - unloading of $0.4 \%$ strain.

To understand the fatigue performance of our SSPs, we first tried cyclic loading - unloading with $0.4 \%$ strain. From Figure 6.4, an increasing trend of resistance change was observed. Limited by the data acquisition method used at the beginning, the obtained data points can only present a rough trend of resistance variation but not the up-and-down shape details of each cycle. This test was repeated with more data points being collected for presentation in the discussion. 


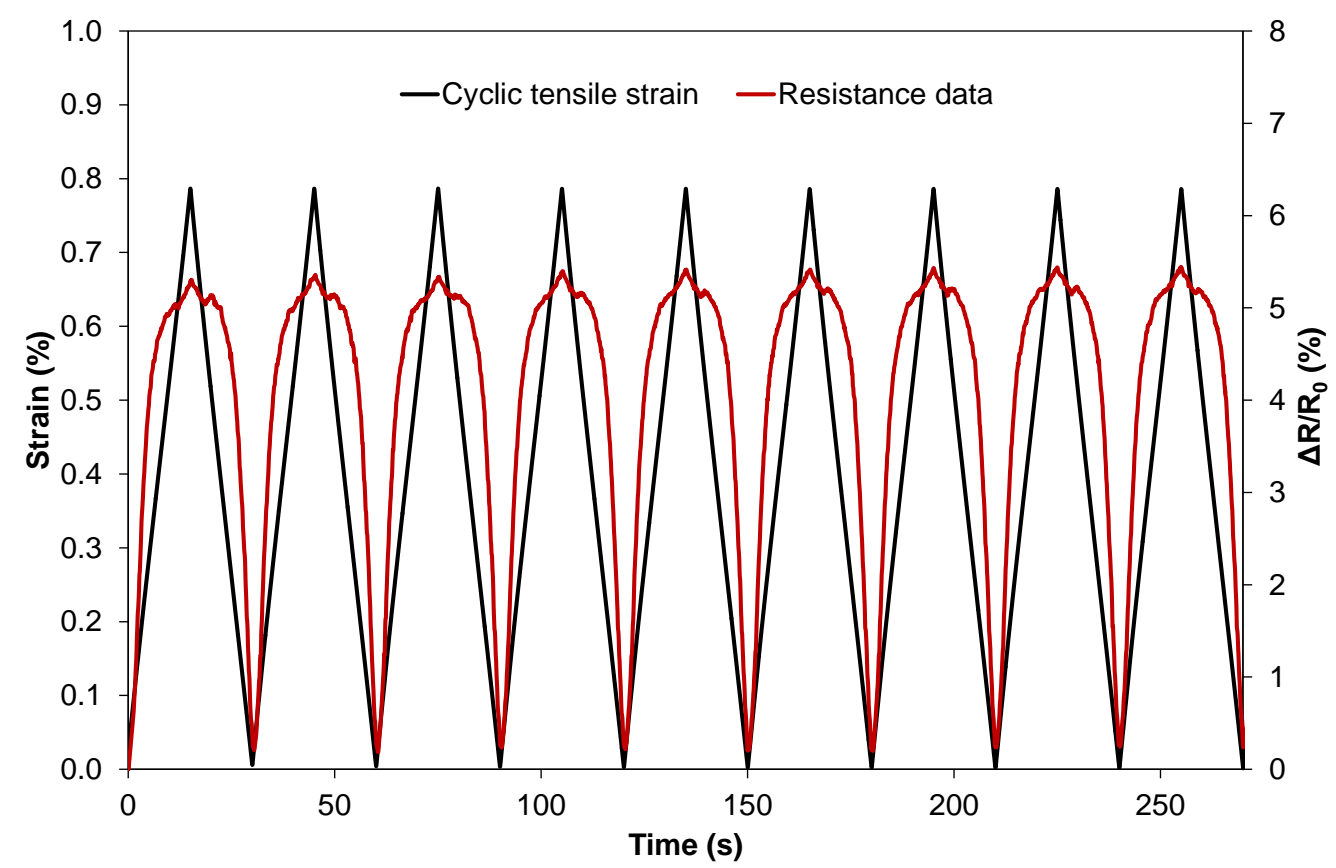

Figure 6.5 Constant strain cyclic tensile test of specimens with tabs: normalized electrical resistance change of parallel SSP versus time in response to 10 cycles of loading - unloading of $0.8 \%$ strain. (After dynamic fatigue testing)

After 1000-cycle dynamic testing, the intrinsic structure of our SSP was believed to be degraded. The resistance change curve can no longer follow the strain variation in a linear manner but behaving in a chapel liked shape. The value of resistance change was observed fluctuating in a larger range, showing a higher sensitivity to strain variation. Moreover, the resistance variation showed good stability and consistency over multiple cycles. 


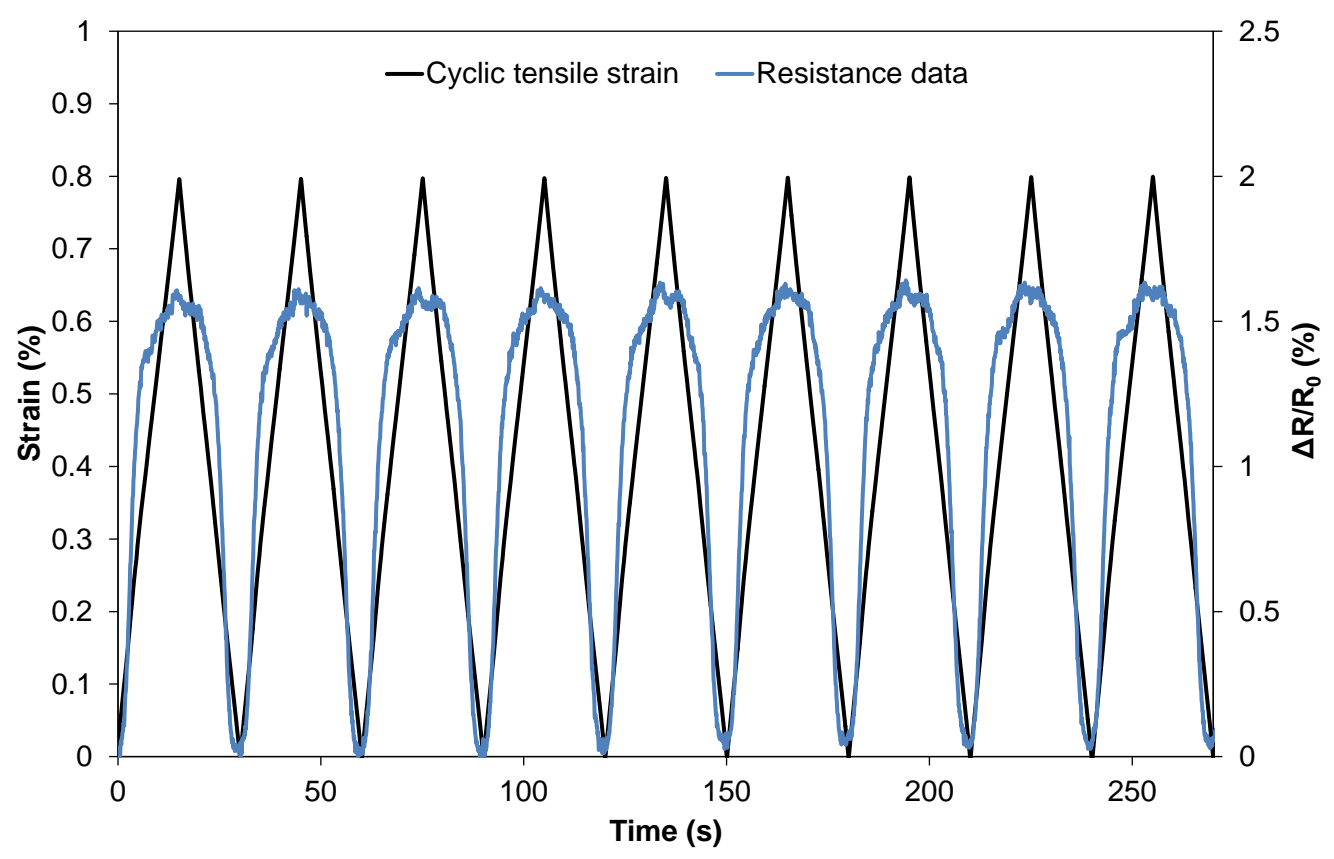

Figure 6.6 Constant strain cyclic tensile test of specimens with tabs: normalized electrical resistance change of perpendicular SSP versus time in response to loading unloading of $0.8 \%$ strain. (After dynamic fatigue testing)

As tested after the 1000-cycle dynamic fatigue test, the perpendicular SSP showed similar behavior to the parallel ones. Chapel shape piezoresistive curve can be observed clearly with higher sensitivity, less noise effect and also improved repeatability. 